\title{
Translational research on Myology \& Mobility Medicine: 2021 semi- virtual PDM3 from Thermae of Euganean Hills, May 26 - 29, 2021
}

\author{
Ugo Carraro (1, 2, 3), Zipora Yablonka-Reuveni (4)
}

(1) Department of Biomedical Sciences of the University of Padova, Italy; (2) CIR-Myo Myology Centre, University of Padova, Italy; (3) A\&C Mioni-Carraro Foundation for Translational Myology, Padova, Italy; (4) Department of Biological Structure, University of Washington School of Medicine, Seattle, WA, USA.

This article is distributed under the terms of the Creative Commons Attribution Noncommercial License (CC BY-NC 4.0) which permits any noncommercial use, distribution, and reproduction in any medium, provided the original author(s) and source are credited.

\begin{abstract}
On 19-21 November 2020, the meeting of the 30 years of the Padova Muscle Days was virtually held while the SARS-CoV-2 epidemic was hitting the world after a seemingly quiet summer. During the 2020-2021 winter, the epidemic is still active, despite the start of vaccinations. The organizers hope to hold the 2021 Padua Days on Myology and Mobility Medicine in a semivirtual form (2021 S-V PDM3) from May 26 to May 29 at the Thermae of Euganean Hills, Padova, Italy. Here the program and the Collection of Abstracts are presented. Despite numerous world problems, the number of submitted/selected presentations (lectures and oral presentations) has increased, prompting the organizers to extend the program to four dense days.
\end{abstract}

Key Words: Padua Muscle Days; skeletal muscle; COVID-19; Translational Myology and Mobility Medicine; 2021 PDM3 Program \& Abstracts.

Eur J Transl Myol 2021; 31 (1): 9743. Doi: 10.4081/ejtm.2021.9743

Meetings on muscle biology, physiology, medicine and rehabilitation, the Padua Muscle Days (PMDs), started more than 30 years ago to provide expert advices on Translational Myology and Mobility Medicine. ${ }^{1-3}$ The aims were on prevention, managing and rehabilitation of mobility disorders. Last year the organizers programmed for March 2020 an intense 3-day program to be held either at Padova University or at the Hotel Augustus, Thermae of Euganean Hills (Padova), Italy. ${ }^{4}$ Abruptly, in January 2020 the SARS CoV-2 outbreak changed the world perspectives. In Italy the virus was first detected in Lombardy and Veneto. Within a week, the COVID-2019 affected the first Italian victims in an area south of Milan and in a village of the Euganean Hills (Vo Euganeo, Padova). The village was immediately quarantined, but it was too late. The virus was spreading through the area. Thus, the PMDs were postponed and successfully held as Virtual ZOOM Series of Presentations from 19 to 21 November, 2020. Luckily, chairs, speakers and attendees accepted the decisions. ${ }^{5}$

In 2021 COVID-19 epidemic is still active, despite vaccinations. The organizers hope to hold the 2021 Padua Days on Myology \& Mobility Medicine (PDM3) semivirtually form May 26 to May 29 at the Thermae of Euganean Hills, Padova, Italy. Here a preliminary
Program of Sessions and the Collection of Abstracts follow. Despite the debilitating problems, the number of selected presentations (lectures and oral presentations) has increased to fill in an extend program of four dense days. Beside the traditional sessions, the Program presents several new topics: Extracellular Matrix, Fasciae \& Tendons; Muscle and Sarcomeres in vivo; High density EMG and motor control in health and disease; Mechanisms of Skeletal Muscle Impairment in Aging and Disease; Muscle Atrophy, Sarcopenia, Cachexia: Physical, Nutritional, and Pharmacological Interventions; Light and Electron Microscopy Muscle Imaging; Satellite Cells and Muscle Regeneration: Biology \& Pathology; Therapy of Genetic Muscle Dystrophies; Muscle Rehabilitation: Balneology \& Balneotherapy; Muscle Rehabilitation: Clinical Aims \& Case Reports; Muscle Rehabilitation: Roles of Mitochondria \& Excitation-Contraction Coupling. We hope 2021PDM3 will be as successful as the events of the previous 30 years. The Speakers will be double the usual, imposing an extension to four days. It is hoped that all Lectures, Speakers and Attendees will patiently wait for the next difficult step: reschedule the time slots to allow the interactive presence of people who will participate from time zones distributed over 24 hours. It will be a difficult task, but we will succeed. 


\section{TRANSLATIONAL RESEARCH ON MYOLOGY \& MOBILITY MEDICINE} May 26 - 29, 2021 - Thermae of Euganean Hills (Padova), Italy
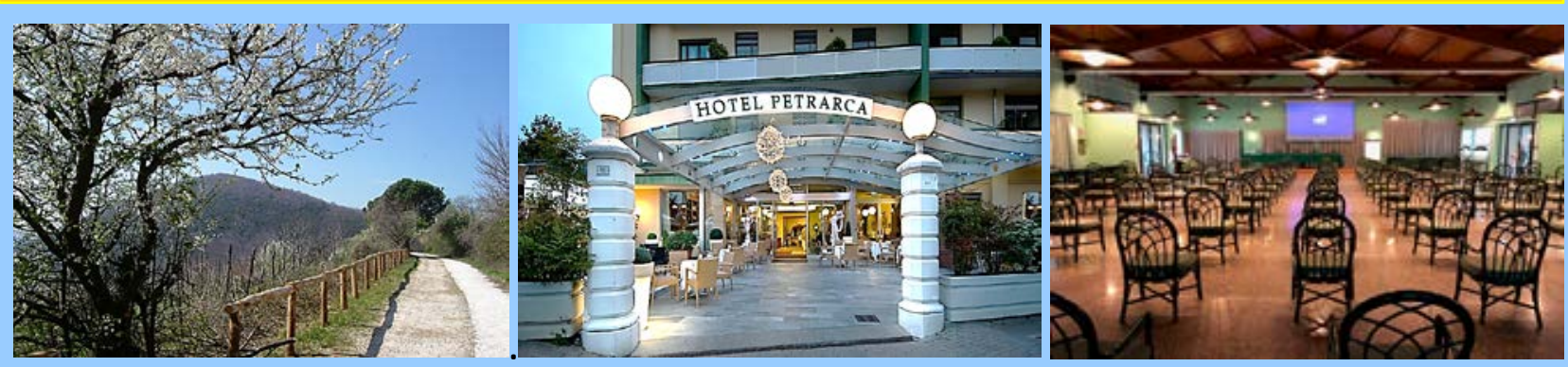

WEDNESDAY May 26, 2021

9:30 AM Openings

10:00 AM Session I: Extracellular Matrix, Fasciae \& Tendons

3:00 PM Session II: Muscle \& Sarcomeres in vivo

5:20 PM Session III: High density EMG applied to motor control in health \& disease

\section{THURSDAY May 27, 2021}

9:00 AM Session IV: Poster Presentations

11:00 AM Session V: Muscle Atrophy, Sarcopenia, Cachexia: Physical, Nutritional \& Pharmacological Interventions

3:00 PM Session VI: Mechanisms of Skeletal Muscle Impairment in Aging \& Disease

5:20 PM Session VII: Light \& Ultrastructural Muscle Imaging

FRIDAY May 28, 2021

9:00 AM Session VIII: Poster Presentations

10:30 AM Session IX: Satellite Cells \& Muscle Regeneration: Biology \& Pathology

3:00 PM Session X: Satellite Cells \& Muscle Regeneration: Biology \& Pathology

5:20 PM Session XI: Therapy of Genetic Muscle Dystrophies

SATURDAY May 29, 2021

9:00 AM Session XII: Muscle Rehabilitation: Balneology \& Balneotherapy

11:30 AM Session XIII: Muscle Rehabilitation: Clinical Aims \& Case Reports

3:00 PM Session XIV: Muscle Rehabilitation: Roles of Mitochondria \& E-C Coupling

5:00 PM GENERAL DISCUSSION OF THE CENTER FOR ACTIVE AGING GROUP

7:30 PM Ugo Carraro: See you to the 2022 PDM3, March, 2022-Thermae of Euganean Hills \& Padova, Italy

\section{Translational Research on Myology \& Mobility Medicine}

Call for EJTM COMMUNICATIONS - EJTM 31 (2), 2021 - Deadline May 31, 2021

(2500 words, 150-word Abstract, 5 keywords, 4 Figures/Tables, 30 Refs).

EJTM is indexed in PUBMED, ESCI and SCOPUS - CiteScoreTracker 2020 = 2.00

Submit to: pagepressjournals.org/index.php/bam/about/submissions 


\section{Semi-Virtual PDM3 - May 26 to29}

Thermae of Euganean Hills (Padova), Italy, May 26 - 29, 2021

Hotel Petrarca, Piazza Roma 23, Montegrotto Terme, Thermae of Euganean Hills, (Padova), 35122 Italy

Phone +39049891 1744 - Email: petrarca@hotelpetrarca.it - https://www.hotelpetrarca.it/

Organizers: Ugo Carraro, Helmut Kern, Christiaan Leeuwenburgh, Marco Narici, Marco Sandri, Carla Stecco, Zipora Yablonka-Reuveni

\section{Collection of Abstracts}

\section{\#1. May 26, 2021}

Invited Lecture: Physical exercise and adaptation of connective tissue in tendon and muscle

\section{Michael Kjaer}

(1) Institute of Sports Medicine, Dept. Orthopedic Surgery, Copenhagen University Hospital - Bispebjerg, and Center for Healthy Aging, Faculty of Health and Medical Sciences, University of Copenhagen, Denmark

Michael Kjaer: michaelkjaer@sund.ku.dk

The overall turnover of the tendon and cartilage in humans seems to be taking place primarily within the first 13-17 years of life (markedly lower than turnover of contractile protein in muscle), indicating that the major basic structure remains relatively unchanged through adult life. Nevertheless, mechanical loading of adult human tendon (and intramuscular connective tissue) results in tendon cell responses by producing anabolic growth factors and loading-induced increase in tendon collagen synthesis. Comparing tissue turnover in different tissues simultaneously suggests that a combination of a more basic structure that remains relatively unchanged through adult life, and a smaller pool of collagen that is more quickly turned over and can be influenced by mechanical loading. Conversely, physical inactivity down regulates collagen synthesis and phenotypic tendon characteristics. Adjustment of the tendon mechanical properties in the form of increased stiffness and modulus after strength training, and the reverse after period of immobilization occurs relatively faster than macroscopic morphological changes of tendon. Age related changes in tendon connective tissue with reduced stiffness is largely explained by a reduction in overall physical activity and can thus be at least partly counteracted by regular training.

Keywords: Physical exercise; adaptation; connective tissue; tendon; muscle.

\section{References}

1. Agergaard AS, Svensson RB, Malmgaard-Clausen NM, Couppé C, Hjortshoej MH, Doessing S, Kjaer M, Magnusson SP.Clinical Outcomes, Structure, and Function Improve With Both Heavy and Moderate Loads in the Treatment of Patellar Tendinopathy: A Randomized Clinical Trial. Am J Sports Med. 2021 Feb 22:363546520988741. doi: 10.1177/03635465 20988741. Online ahead of print.

\section{\#2. May 26, 2021}

Effect of aging on tendon mechanical properties and adaptability to chronic loading

\section{Marco Narici \\ CIR-Myo Myology Centre, Neuromuscular Physiology Laboratory, Department of Biomedical Sciences, University of Padova, Padova, Italy}

* Marco Narici: marco.narici@unipd.it

Tendons play a fundamental role in transmitting forces generated by skeletal muscle and in acting as springs during locomotion. Because of their anatomical arrangement, the mechanical properties of tendons affect those of skeletal muscle, above all, the length-force relation., ${ }^{1,2}$ With ageing, human tendon mechanical properties (stiffness, Young's modulus and hysteresis) seem to deteriorate, ${ }^{3-6}$ although reports of no changes also exist. ${ }^{7}$ These alterations potentially contribute to the loss of muscle force with ageing. ${ }^{8}$ However, tendons retain considerable plasticity in response to chronic loading in old age since their mechanical properties can be substantially improved by high-intensity resistance exercise training (RET). Indeed, after 14 weeks of RET in septuagenarian males, patellar tendon stiffness and Young's modulus and hysteresis were found to improve by $65 \%$ and $69 \%$ respectively, restoring tendon properties to values comparable to those of young adults. $^{9,10}$ These improvements were mostly due to changes in tendon tissue material properties rather than to tendon hypertrophy. Also, the increase in tendon stiffness was associated with a faster rate of force development, a finding of particular relevance for falls prevention. While traditional RET consists of both concentric (CON) and eccentric (ECC) contractions, ECC contractions are metabolically less demanding and therefore may be more suitable for an older population. Thus, we investigated the tendon adaptations and time course, to 8-week, 3 times/week, submaximal (60\% $1 \mathrm{RM}$ ) CON only or ECC only RET in 20 healthy young (24.5 $\pm 5.1 \mathrm{yrs})$ and 17 older males $(68.1 \pm 2.4 \mathrm{yrs})$. Patellar tendon (PT) Young's modulus and stiffness were assessed every 2 weeks. Changes in PT Young's modulus were observed after only 4 weeks in all groups. Importantly, both CON and ECC resulted in similar changes of PT Young's modulus at week 8, in both young and older males indicating that tendons are 'blind' to the direction of muscle loading (11). Notably, changes in tendon tissue seemed to occur in a coordinated fashion 
with those of skeletal muscle; presumably in an effort to maintain the efficacy of the muscle-tendon unit. These findings demonstrate that despite the effects of aging, tendons retain considerable adaptability to chronic loading, recovering material properties comparable to those of young healthy adults.

Keywords: tendon; skeletal muscle; training; aging.

\section{References}

1. Lichtwark GA, Wilson AM. Is Achilles tendon compliance optimised for maximum muscle efficiency during locomotion? J Biomech. 2007;40(8):1768-75

2. Magnusson SP, Narici MV, Maganaris CN, Kjaer $M$. Human tendon behaviour and adaptation, in vivo. J Physiol. 2008 Jan 1;586(1):71-81.

3. Onambele GL, Narici MV, Maganaris CN. Calf muscle-tendon properties and postural balance in old age. J Appl Physiol. 2006 Jun;100(6):2048-56.

4. Stenroth L, Peltonen J, Cronin NJ, Sipilä S, Finni T. Age-related differences in Achilles tendon properties and triceps surae muscle architecture in vivo. J Appl Physiol. 2012 Nov;113(10):1537-44.

5. Svensson RB, Heinemeier KM, Couppé C, Kjaer $M$, Magnusson SP. Effect of aging and exercise on the tendon. J Appl Physiol. 2016 Dec 1;121(6):12371246.

6. Quinlan JI, Maganaris CN, Franchi MV, Smith K, Atherton PJ, Szewczyk NJ, Greenhaff PL, Phillips BE, Blackwell JI, Boereboom C, Williams JP, Lund J, Narici MV. Muscle and Tendon Contributions to Reduced Rate of Torque Development in Healthy Older Males. J Gerontol A Biol Sci Med Sci. 2018 Mar 14;73(4):539-545.

7. Couppé C, Hansen P, Kongsgaard M, Kovanen V, Suetta C, Aagaard P, Kjaer M, Magnusson SP. Mechanical properties and collagen cross-linking of the patellar tendon in old and young men. J Appl Physiol. 2009 Sep;107(3):880-6.

8. Narici MV, Maganaris CN. Plasticity of the muscletendon complex with disuse and aging. Exerc Sport Sci Rev. 2007 Jul;35(3):126-34

9. Reeves ND, Maganaris CN, Narici MV. Effect of strength training on human patella tendon mechanical properties of older individuals. $J$ Physiol. 2003 May 1;548(Pt 3):971-81.

10. Reeves ND, Narici MV, Maganaris CN. Strength training alters the viscoelastic properties of tendons in elderly humans. Muscle Nerve. 2003 Jul;28(1):74-81.

11. Quinlan JI, Franchi MV, Gharahdaghi N, Badiali $F$, Phillips BE, Greenhaff P, Szewczyk N, Atherton PJ, Smith K, Maganaris CN, Narici MV (2019). The time course of tendon and muscle adaptations to moderate-load eccentric vs concentric resistance exercise in young and older males. In Proc. 24th Annual ECSS Congress Prague/Czech Republic, July 3-6 2019.

\section{\#3. May 26, 2021}

Effects of Age on Extracellular Matrix of Muscle Spindle in Skeletal Muscle

Chenglei Fan (1), Caterina Fede (1), Carmelo Pirri (1), Diego Guidolin (1), Carlo Biz (2), Lucia Petrelli (1), Andrea Porzionato (1), Veronica Macchi (1), Raffaele De Caro (1), Carla Stecco (1)*

(1) Department of Neuroscience, Institute of Human Anatomy, University of Padua, Padua, Italy; (2) Department of Surgery, Oncology and Gastroenterology, Orthopedic Clinic, University of Padua, Padua, Italy

\section{* Carla Stecco: carla.stecco@unipd.it}

Muscle spindle (MS), as a stretching receptor, plays a crucial role in proprioception and locomotor coordination in musculoskeletal system. ${ }^{1}$ They are very sensitive to stretch, feeling a tension of few grams. For that reason, the elasticity and viscosity of the extracellular matrix (ECM) where MSs are placed could play a key role to define their sensitivity to movements. ${ }^{2}$ It is well known that their sensitivity decreases with aging, causing postural sway and gait stability deterioration, ${ }^{3,4}$ but the relationships, between the extracellular ECM where MSs are placed and aging, are unclear. Therefore, the aim of this study was to investigate whether the contents of ECM where MSs are placed change with aging, to better understand if age-related physiological changes of muscle spindles could be due also to an alteration of the environment where they are located, and not only to neurodegeneration. Hematoxylin Eosin, Picrosirius-red, collagen type I (COLI) and III (COLIII) antibodies, and biotinylated hyaluronan binding protein (HABP) immunohistochemistry staining were used to evaluate alterations in intramuscular connective tissue (ICT) around the MS and of the ECM of MS itself in 6-weeks puberty (group A), 8-months middle age (group B) and 2 years old (group C) C57BL/6J male mice hind limb muscles. All the MS capsules are continuous with the perimysium of the skeletal muscle. The outer layer of capsule becomes thicker with aging, passing from3.02 $\pm 0.26 \mu \mathrm{m}$ in group A to $3.64 \pm 0.31 \mu \mathrm{m}$ in group B and $5.81 \pm 0.85 \mu \mathrm{m}$ in group C. The capsules of the MSs and the ICT mainly comprise COLI and COLIII. The collagen content in whole muscle and inside MS was significantly higher in the group $\mathrm{C}$, whereas there were no significantly differences of these parameters between group A and group B. The average optical density (AOD) of COLI in the MS was significantly increased with aging $(\mathrm{P}<0.05)$, whereas there were no significant differences of COLIII among the three groups $(\mathrm{P}>0.05)$. In addition, HA is present in the ICT and fills the capsule of MS. The AOD of the HABP immunohistochemistry staining of the MS highlights a decreasing of HA in group $\mathrm{C}$ compared to group $\mathrm{A}(\mathrm{P}=0.022)$, whereas, there were no significant difference between group $\mathrm{A}$ and group $\mathrm{B}$ and between 
group $\mathrm{B}$ and group $\mathrm{C}(\mathrm{P}>0.05)$. The accumulation of collagen content and decreased HA in ECM where MSs are placed could change the adaptability of ICT to stretch and movements, reducing the sensitivity of MS to dynamic and static lengthening. These alterations of ECM where MSs are placed could help to explain the peripheral mechanisms of the decline of age-related proprioception and locomotor coordination.

Keywords: aging, extracellular matrix, muscle spindle, collagen, collagen type I, collagen type III, hyaluronan, connective tissue.

\section{References}

1. Windhorst U. Muscle proprioceptive feedback and spinal networks. Brain Res Bull. 2007 Jul 12;73(46):155-202. doi: 10.1016/j.brainresbull.2007.03. 010. Epub 2007 Apr 17.

2. Stecco A, Stecco C., Raghavan P. Peripheral mechanisms contributing to spasticity and implications for treatment. Current Physical Medicine and Rehabilitation Reports 2014;2:121127.

3. Miwa T, Miwa Y, Kanda K.. Dynamic and static sensitivities of muscle spindle primary endings in aged rats to ramp stretch. Neurosci Lett. 1995 Dec 8;201(2):179-82. doi: 10.1016/0304-3940(95) 12165- $x$

4. Pavan P, Monti E, Bondí M, Fan C, Stecco C, Narici M, Reggiani C, Marcucci L. Alterations of extracellular matrix mechanical properties contribute to age-related functional impairment of human skeletal muscles. Int J Mol Sci. 2020 Jun 2;21(11):3992. doi: 10.3390/ijms21113992.

$$
* * * * *
$$

\#4. May 26, 2021

An experimentally-based finite element model for estimating the loss of force due to impaired lateral force transmission in older humans

Lorenzo Marcucci,* Silvia Todros (2), Marco Narici (1), Carlo Reggiani (1), Piero Pavan (2)

(1) Department of Biomedical Science, University of Padova, Italy; (2) Department of Industrial Engineering, University of Padova, Italy

* Lorenzo Marcucci: lorenzo.marcucci@unipd.it

The age-related loss of joint mobility (LJM) sets limitations to independent life and increases the risk of falls and hospitalization, with a strong impact on life conditions and social costs ${ }^{1}$. One of the major causes of LJM is muscle weakness, which is associated to functional impairment and loss of independence and is recognized as an independent risk factor for increased mortality of older people. In this respect, a striking phenomenon is that the decrease of muscle mass with aging (sarcopenia) is accompanied by a much greater decline in muscle strength (dynapenia). Several studies suggest that the greater decline of muscle force compared to muscle mass is associated with a decreased ability to laterally transmit the force generated by muscle fibers ${ }^{2}$. This effect, which reduces the efficiency force transmission at the macroscopic level, is generated, at least partially, by alterations of the extra-cellular matrix (ECM) with aging. ECM is a hierarchical structure that surrounds single fibers (endomysium), fascicles (perimysium) and the whole muscle (epimysium). Therefore, to quantitatively assess the role of this alteration at any level, multiscale finite element modelling is a powerful tool. However, experimental data collection is a crucial step to implement modeling and achieve reliable predictions. To this aim we compared, in a recent work $^{3}$, the relative contribution of the ECM to passive tension and stiffness in small bundles in young healthy (Y: 21 years) and older (E: 67 years) males. We showed that the resting passive tension is significantly higher in the bundles of the older compared to those of the younger males, while no significant difference was found in single fibers. The difference was then attributed to the ECM present between the fibers composing the bundle. However, our data suggested that the larger amount of collagen present in older males (3.3\% in the younger and $8.2 \%$ in older males) can fully account for the observed difference in total tension, and that the intrinsic stiffness of the connective material was almost unchanged or even more compliant in older individuals. ECM Young's modulus was $580 \mathrm{kPa}$ for the older males and $809 \mathrm{kPa}$ for the younger males. To assess how the active force development is affected by the agedependent changes observed in resting conditions, we developed a micromechanical multiscale finite element (FE) model. The model simulates a muscle bundle formed by a few fibers connected through the ECM. Importantly, the model uses separated meshes for the connective tissue and the contractile material and specific assumptions for the links between the meshes at the border between ECM and muscle fibers. Using our experimentally-based parameters, we can quantitatively assess the influence of ECM age-related modifications on lateral force transmission. In detail, we reproduced three classical experimental evidences ${ }^{4-6}$ supporting the contribution of lateral force transmission during contraction, and analyzed the results using the parameters for both younger and older men. The data obtained provide clear evidence of the contribution of changes of ECM to the loss of contractile force with aging.

Keywords: aging; skeletal muscle; resting force; active force; extracellular matrix; finite element model.

\section{References}

1. Marcucci L, Reggiani C. Increase of resting muscle stiffness, a less considered component of age-related skeletal muscle impairment. Eur J Transl Myol. 2020 Jun 17;30(2):8982. doi: 10.4081/ejtm.2019.8982. eCollection 2020 Jul 13. 
2. Csapo R, Gumpenberger M, Wessner B. Skeletal Muscle Extracellular Matrix - What Do We Know About Its Composition, Regulation, and Physiological Roles? A Narrative Review. Front Physiol. 2020 Mar 19;11:253. doi: 10.3389/fphys. 2020.00253.

3. Pavan P, Monti E, Bondí M, Fan C, Stecco C, Narici M, Reggiani C, Marcucci L. Alterations of Extracellular Matrix Mechanical Properties Contribute to Age-Related Functional Impairment of Human Skeletal Muscles. Int J Mol Sci. 2020 Jun 2;21(11):3992. doi: 10.3390/ijms21113992.

4 Huijing PA. Muscle as a collagen fiber reinforced composite: a review of force transmission in muscle and whole limb. J Biomech. 1999 Apr;32(4):329-45. doi: 10.1016/s0021-9290(98)00186-9.

5 Ramaswamy KS, Palmer ML, van der Meulen JH, Renoux A, Kostrominova TY, Michele DE, Faulkner JA. Lateral transmission of force is impaired in skeletal muscles of dystrophic mice and very old rats. J Physiol. 2011 Mar 1;589(Pt 5):1195-208. doi: 10.1113/jphysiol.2010.201921. Epub 2011 Jan 10.

6 Street SF. Lateral transmission of tension in frog myofibers: a myofibrillar network and transverse cytoskeletal connections are possible transmitters. J Cell Physiol. $1983 \quad$ Mar;114(3):346-64. doi: 10.1002/jcp.1041140314.

$$
* * * * *
$$

\#5. May 26, 2021

Testing soft tissue radiodensity parameters interplay with age and self-reported physical activity starting from a mid-thigh CT-scan

Carlo Ricciardi (1,2), , Marco Recenti (1), Kyle J. Edmunds (1,3), Anaïs Monet (1), Deborah Jacob (1) Jorgelina Ramos (1), Monica Gambacorta (4), Ugo Carraro (5,6), and Paolo Gargiulo $(1,7)^{*}$

\#Marco Recenti and Carlo Ricciardi contributed equally as co-first authors

(1) Institute for Biomedical and Neural Engineering, Reykjavik University, 102 Reykjavik, Iceland. (2) Department of Advanced Biomedical Sciences, University Hospital of Naples 'Federico II', Naples, Italy. (3) University of Wisconsin School of Medicine and Public Health, Madison, Wisconsin, USA. (4) Umberto I Hospital, ASL Salerno, Nocera Inferiore, Italy. (5) CIRMyo - Myology Centre, Department of Biomedical Sciences, University of Padova, Italy. (6) A\&C MioniCarraro Foundation for translational Myology, Padova, Italy; (7) Department of Science, Landspitali University Hospital, 101 Reykjavik, Iceland.

* Paolo Gargiulo; paologar@landspitali.is

Nonlinear trimodal regression analysis (NTRA) is a recent methodology for modelling the radiodensitometric distributions of x-ray computed tomography (CT) scans, producing 11 patient-specific features which characterize qualitatively and quantitatively the lean muscle, fat, and loose connective tissues. In this paper, the association of these 11 NTRA features with age and physical activity is investigated in the population-based AGES-I dataset ( $\mathrm{n}=$ 3,157 elderly volunteers). First, univariate statistical analyses were performed through IBM SPSS (version 25) using Kruskal Wallis and post-hoc tests, where subjects were grouped by age and self-reported past (youth and mild-life) and present (within 12 months of the survey) physical activity to ascertain which parameters were most influent on the grouping variables. Then, machine learning (ML) analyses were conducted through Knime Analytics Platform (version 4.1.1) to classify patients employing NTRA parameters as input features for three decision tree based ML algorithms (random forests, gradient boosting, and ADA-boosting). This classification analysis yielded poor results (accuracies did not overcome $60 \%$ ), and regression analyses were not particularly reliable or robust. However, univariate statistical models suggested that NTRA parameters may be strongly sensitive to age and differences between past and present physical activity levels. Moreover, for both age and physical activity, lean muscle parameters expressed greater variation, compared with fat and connective tissues. This paper consists in further evidence that radiodensitometric distribution values are sensitive to changes in physiological parameters.

Keywords: Machine learning; CT; soft tissues.

References

1. Edmunds KJ, Árnadóttir Í, Gíslason MK, Carraro U, Gargiulo P. Nonlinear trimodal regression analysis of radiodensitometric distributions to quantify sarcopenic and sequelae muscle degeneration. Comput. Math. Methods Med. Comput Math Methods Med. 2016;2016:8932950. doi: 10.1155/2016 /8932950. Epub 2016 Dec 27.

2. Edmunds K, Gíslason M, Sigurðsson S, Guðnason V, Harris T, Carraro U, Gargiulo P. Advanced quantitative methods in correlating sarcopenic muscle degeneration with lower extremity function biometrics and comorbidities. PLoS One. 2018 Mar 7;13(3):e0193241. doi: 10.1371/journal.pone. 0193241. eCollection 2018.

$$
* * * * *
$$

\#6. May 26, 2021

\section{Effects of Aging on Intramuscular Connective Tissue}

Chenglei Fan (1), Caterina Fede (1), Carmelo Pirri (1), Diego Guidolin (1), Carlo Biz (2), Lucia Petrelli (1), Andrea Porzionato (1), Veronica Macchi (1), Raffaele De Caro (1), Carla Stecco (1)*

(1) Department of Neurosciences, Institute of Human Anatomy, University of Padua, Padua, Italy; (2) Department of Surgery, Oncology and Gastroenterology, Orthopedic Clinic, University of Padua, Padua, Italy

* Carla Stecco: carla.stecco@unipd.it 
Extracellular matrix (ECM) of intramuscular connective tissue (IMCT) play critical role not only in maintaining integrity of muscle, ${ }^{1}$ but also in providing mechanical properties and local signals to modulate muscle cell fusion and regeneration. It is known that the muscles present many degenerative processes with aging. ${ }^{2,3}$ However, the effects of aging in the ECM of IMCT are unclear. Therefore, the aim of this project was to investigate whether the contents of ECM of IMCT change with aging, to better understand the possible effects of age-related ECM alterations of IMCT in the peripheral neuro-pathophysiological mechanisms of locomotor ability deficits. Age-related changes of ECM in human quadriceps femoris were compared in 10 young men (37.5 $\pm 9.0 y), 12$ elderly men $(79.0 \pm 12.4 y)$ and 16 elderly women $(80.6 \pm 11.4 \mathrm{y})$ patients with traumatic fracture (Studio 3027P/AO/13). Age-related ECM alterations in mice hind limb were compared in 6-weeks puberty (group A); 8-months middle age (group B) and 2-years old (group C) C57BL/6J male mice. Hematoxylin Eosin, Picrosirius-red, the collagen type I (COLI), III (COLIII) antibody, and biotinylated hyaluronan binding protein (HABP) immunohistochemistry staining were used to evaluate the morphology, collagen content, COLI, COLIII and HA both in human and mice muscle cross-section. Alcian Blue, Weigert Van Gieson staining were used to evaluate the glycosaminoglycans, elastic fiber, in human male specimens. Age-related alterations of HA concentrations were evaluated both in human and mice specimens using Purple-Jelley HA assay. Muscle loss and fat infiltration were present in most elderly samples, especially in the subjects over 70 years old. The area percentage of collagen contents were significantly elevated in the elderly group compared to young men group $(\mathrm{P}<0.01)$. While, there was no significantly difference between the elderly men and women group in collagen content. The collagen contents in IMCT were significantly increased in mice with aging. To better understand which subtype collagen was more suffered with aging. The following experiments were performed in the male human and mice specimen due to the possible effects of gender (female). The accumulation of the collagen content was mainly due to an increase in COLI both in the human and mice specimens, while there were no significantly differences in COLIII. In addition, the area percentage of elastic fibers in perimysium was significantly lower $(\mathrm{P}<0.01)$ in the elderly men group. The HA was mainly localized at endomysium, perimysium and epimysium. The HA content was significantly decreased in the elderly man compare to the young men $(\mathrm{P}=0.04)$, while, there was no significantly difference between the elderly men and women group in HA. In addition, the amount of HA in mouse hind limb muscle was significantly decreased with health aging (group $\mathrm{A}$ vs group $\mathrm{B}$ : $\mathrm{P}<0.05$; group $\mathrm{A}$ vs group $\mathrm{C}$ : $\mathrm{P}<0.01$ ). The accumulation of collagen content and decreased HA in ECM of IMCT, the decreased relative elastic fibers in perimysium may cause the IMCT stiffer and reduce its' adaptability, changing gliding of the IMCT and influence the function of muscle fibers. These alterations of ECM in IMCT properly can partly explain the peripheral mechanisms of the decline of age-related locomotor ability.

Keywords: aging, extracellular matrix, collagen, elastic fiber, collagen type I, collagen type III, hyaluronan, intramuscular connective tissue

\section{References}

1. Gillies, AR, Lieber RL. Structure and function of the skeletal muscle extracellular matrix. Muscle Nerve. 2011 Sep;44(3):318-31. doi: 10.1002/mus.22094.

2. Kragstrup TW, Kjaer M, MackeyA. Structural, biochemical, cellular, and functional changes in skeletal muscle extracellular matrix with aging. Scand J Med Sci Sports. 2011 Dec;21(6):749-57. doi: 10.1111/j.1600-0838.2011.01377.x.

3. Stearns-Reider KM, D'Amore A, Beezhold K, Rothrauff B, Cavalli L, Wagner WR, Vorp DA, Tsamis A, Shinde S, Zhang C, Barchowsky A, Rando TA, Tuan RS, Ambrosio F. Aging of the skeletal muscle extracellular matrix drives a stem cell fibrogenic conversion. Aging Cell. 2017 Jun;16(3):518-528. doi: 10.1111/acel.12578. Epub 2017 Mar 30.

$$
* * * * *
$$

\#7. May 26, 2021

Biomechanical and structural studies of the muscle extracellular matrix

\section{Richard L. Lieber}

Shirley Ryan AbilityLab, Departments of Physical Medicine, Rehabilitation and Biomedical Engineering, Northwestern University, Chicago, IL, USA

* Richard L. Lieber: rlieber@sralab.org

Passive skeletal muscle mechanical properties are not as well understood as their active contractile properties. Both the structural basis for passive mechanical properties and the properties themselves are challenging to determine because it is not clear which structures within muscle actually bear passive loads and the best way to make mechanical measurements. Early studies showed the clear and important role of titin as a muscle fiber load bearing structure, ${ }^{1}$ but studies comparing single muscle fibers to small fiber bundles suggest that much more load is borne extracellularly. ${ }^{2-4}$ Recently, we have performed more high resolution structural and functional studies of the ECM using three different approaches: confocal microscopy on muscle fiber segments, ${ }^{5}$ three-dimensional reconstruction of electron micrographs, ${ }^{6}$ and traditional stereology. ${ }^{5}$ These studies reveal the presence of perimysial cables that generally extend parallel to muscle fibers. Studies using the desmin knockout mouse model of fibrosis, ${ }^{7,8}$ also demonstrate that the number of cables as well as their size appear to 
be regulated. Finally, the cellular basis of fibrosis in the ECM was investigated using a transgenic mouse model in which Type I collagen producing cells were labeled with GFP. ${ }^{9}$ These studies revealed that muscle satellite cells, fibroblasts and fibroadipogenic progenitor cells all appear to participate in the fibrotic response. We suggest that new high resolution methods are needed at larger scales as well as a standardized set of rules for the reporting of passive mechanics to allow cross-study comparisons. Such studies are required to develop therapies to treat disorders in which passive muscle properties are altered in conditions such as muscular dystrophy, traumatic laceration, and contracture due to upper motor neuron lesion as seen in spinal cord injury, stroke and cerebral palsy.

Keywords: fibrosis, modulus, collagen, perimysium, extracellular matrix

\section{References}

1. Magid A, Law DJ. Myofibrils bear most of the resting tension in frog skeletal muscle. Science (Washington DC). 1985;230:1280-2.

2. Meyer $G$, Lieber RL. Muscle fibers bear a larger fraction of passive muscle tension in frogs compared with mice. J Exp Biol. 2018;221(Pt 22). Epub 2018/09/22. doi: 10.1242/jeb.182089. PubMed PMID: 30237238; PMCID: PMC6262763.

3. Meyer GA, Lieber RL. Elucidation of extracellular matrix mechanics from muscle fibers and fiber bundles. Journal of Biomechanics. 2011;44(4):7713. Epub 2010/11/26. doi: S0021-9290(10)00610-X [pii] 10.1016/j.jbiomech.2010.10.044. PubMed PMID: 21092966; PMCID: 3042517.

4. Ward SR, Winters TM, O'Connor SM, Lieber RL. Nonlinear scaling of passive mechanical properties in fibers, bundles, fascicles and whole rabbit muscles. Frontiers in Physiology. 2020. doi: doi.org/10.3389/fphys.2020.00211.

5. Gillies AR, Chapman MA, Bushong EA, Deerinck TJ, Ellisman $M H$, Lieber $R L$. High resolution threedimensional reconstruction of fibrotic skeletal muscle extracellular matrix. J Physiol. 2017;595(4):115971. Epub 2016/11/20. doi: 10.1113/JP273376. PubMed PMID: 27859324; PMCID: PMC5309386.

6. Gillies AR, Bushong EA, Deerinck TJ, Ellisman MH, Lieber $R L$. Three-dimensional reconstruction of skeletal muscle extracellular matrix ultrastructure. Microsc Microanal. 2014;20(6):1835-40. doi: 10.1017/S1431927614013300. PubMed PMID: 25275291; PMCID: 4267978.

7. Li Z, Mericskay M, Agbulut O, Butler-Browne G, Carlsson L, Thornell LE, Babinet C, Paulin D. Desmin is essential for the tensile strength and integrity of myofibrils but not for myogenic commitment, differentiation, and fusion of skeletal muscle. Journal of Cell Biology. 1997;139(1):12944. Epub 1997/10/06. PubMed PMID: 9314534; PMCID: 2139820.
8. Meyer GA, Lieber RL. Skeletal muscle fibrosis develops in response to desmin deletion. Am J Physiol Cell Physiol. 2012;302(11):C1609-20. doi: 10.1152/ajpcell.00441.2011. PubMed PMID: 22442138; PMCID: 3378016.

9. Chapman MA, Mukund K, Subramaniam S, Brenner $D$, Lieber RL. Three Distinct Cell Populations Express Extracellular Matrix Proteins and Increase in Number During Skeletal Muscle Fibrosis. Am J Physiol Cell Physiol. 2017;312(2):C131-C43. Epub 2016. doi: 10.1152/ajpcell.00226.2016. PubMed PMID: 27881411

\#8. May 26, 2021

\section{Sarcomere remodeling and function in ageing muscle}

Geoffrey A. Power (1,2), Sean Crooks (2), Jared R. Fletcher (2,3), Brian R. Macintosh (2), Walter Herzog (2)

(1) Department of Human Health and Nutritional Sciences, College of Biological Sciences, University of Guelph, Guelph, Ontario, Canada; (2) Human Performance Laboratory, Faculty of Kinesiology, University of Calgary, Calgary, Alberta, Canada; (3) Department of Health and Physical Education, Mount Royal University, Calgary, Alberta, Canada

* Geoffrey Power: gapower@uoguelph.ca

In old age, there appears to be elevated passive tension for a given muscle length ${ }^{1}$. Aging muscles undergo structural remodeling, whereby muscle fascicle lengths become shorter in old as compared with young ${ }^{2,3}$. Thus, the fascicles and sarcomeres of muscles in older adults may experience greater relative length changes for a given displacement or joint angular rotation, resulting in increased passive tension as compared with young. The purpose of this study was to compare the medial gastrocnemius of young and old rats to determine differences in fascicle length, serial sarcomere numbers and the sarcomere length at which peak force is obtained (optimal reference length; RL), and record passive tension from short to long muscle lengths. Two groups of Fisher344x BN rats were used in this experiment, a young cohort $(\approx 9$ months $\approx 20$ human years $)$ and old cohort ( $\sim 32$ months; $\approx 75-80$ human years). The MG from the right leg was surgically isolated, attached to a custom made muscle puller and force transducer. Passive force was recorded at nine muscle lengths relative to the length producing optimal active force. Following passive force and length measurements, the animals were sacrificed and the hind limb was fixed in formalin at the muscle length corresponding to optimal force. The muscles were then dissected into four lengthwise sections medial and lateral of the center and sarcomere length measurements ${ }^{4}$ were obtained. A reduction in muscle fascicle length of $\sim 14 \%$ was observed in the old rats when compared to young $(\mathrm{p}<0.05)$ and the old rats had $\sim 10 \%$ fewer sarcomeres in series when compared to young rats 
$(p<0.05)$. There was no difference in sarcomere length at optimal length for force production $(p>0.05)$. While there was no difference in passive tension at short muscle lengths between groups, old rats experienced up to $\sim 125 \%$ greater passive tension at long muscle lengths as compared with young $(\mathrm{p}<0.05)$. We show the ageassociated reduction in muscle fascicle length is primarily owing to fewer sarcomeres arranged in series, without a significant difference in sarcomere length. Additionally, passive force was greater in muscles from the old rats as compared with young at long muscle lengths. It was expected that shorter fascicles and fewer serial sarcomeres in muscles from old experienced greater relative length changes for a given displacement, resulting in increased passive tension at long muscle lengths as compared with young. However, the $\sim 4 \%$ shorter sarcomere lengths in muscles from old, as compared with young may have dampened the effects of shorter fascicles in old minimizing any 'overstretching' at long lengths. For the absolute length change, on average, fascicles from young were stretched $\sim 30 \%$ while old were stretched $\sim 36 \%$, meaning muscles from both young and old were stretched to an average sarcomere length of $\sim 3.01 \mu \mathrm{m}$. Therefore, in the present study, differences in sarcomere length at the long muscle length are likely not the explanation for the age-related differences in passive force. Funding by NSERC is acknowledged.

Keywords: aging; sarcomerogenesis; force.

\section{References}

1. Marcucci L, Reggiani C. Increase of resting muscle stiffness, a less considered component of age-related skeletal muscle impairment. Eur J Transl Myol. 2020 Jun 17;30(2):8982. doi: 10.4081/ejtm.2019.8982.

2. Hooper AC. Length, diameter and number of ageing skeletal muscle fibres. Gerontology. 1981;27(3):1216. doi: 10.1159/000212459.

3. Narici MV, Maganaris CN, Reeves ND, Capodaglio P.J. Effect of aging on human muscle architecture. $J$ Appl Physiol. 2003; 95(6), 2229-34. doi: 10.1152/japplphysiol.00433.2003.

4. Lieber RL, Yeh Y, Baskin RJ. Sarcomere length determination using laser diffraction. Effect of beam and fiber diameter. Biophys J. 1984 May;45(5):100716. doi: 10.1016/S0006-3495(84)84246-0..

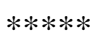

\#9. May 26, 2021

\section{Muscle and sarcomere plasticity in use and disuse}

Martino Franchi (1), Geoffrey A. Power (2), Glen A. Lichtwark (3)

(1) Department of Biomedical Sciences, University of Padova, Italy; (2) Department of Human Health and Nutritional Sciences, College of Biological Sciences, University of Guelph, Canada; (3) School of Human
Movement and Nutrition Sciences, University of Queensland, Australia

\section{* Martino Franchi: martino.franchi@unipd.it}

It is canonically thought that muscle adaptations to loading and unloading are governed by the addition and subtraction of sarcomeres, respectively, both placed in parallel or in series. If we consider muscle fiber hypertrophy, fiber radial growth is mainly governed by an addition of in-parallel material, whereas longitudinal fiber growth is dependent on new sarcomeres added inseries. For these reasons, serial sarcomerogenesis has been studied since the 1960s to gain insight into the mechanisms that regulate muscle plasticity and adaptations to different mechanical stimuli. Interestingly, a majority of the early reports (work of Goldspink, Williams, Tabary and Tardieu, etc, in the 70's and 80's) were obtained in animal models after/during immobilization (long vs. short muscle lengths), indeed showing distinct adaptations in serial sarcomere length and number. ${ }^{1,2}$ Specifically, it seems that when adult animals are exposed to immobilization stimulus at shorter muscle lengths, a loss of serial sarcomere number occurs. Conversely, when immobilization is performed at stretched muscle lengths, an addition of sarcomeres in series is observed. However, no study investigating sarcomerogenesis has been currently performed on humans apart from a single case study (Vastus Lateralis distraction after femur reconstruction) ${ }^{3}$ and animal studies are somehow contradictory, as they present data of presence or absence of sarcomerogenesis but in different species, muscles, conditions, intervention duration. ${ }^{4,5}$ The purpose of this talk will be to present an overview of the main evidence of structural adaptations at whole muscle and sarcomere level to use and disuse. In addition, evidence of the use of novel techniques to gain insight into human sarcomeres in-vivo will be discussed. 6,7

Keywords: sarcomere; plasticity; loading; unloading; sarcomerogenesis.

\section{References}

1. Williams PE, Goldspink G. Longitudinal growth of striated muscle fibres. J Cell Sci. 1971 Nov;9(3):75167.

2. Tabary JC, Tabary C, Tardieu C, Tardieu G, Goldspink G. Physiological and structural changes in the cat's soleus muscle due to immobilization at different lengths by plaster casts. J Physiol. 1972 Jul;224(1):231-44. doi: 10.1113/jphysiol.1972.sp 009891.

3. Boakes JL, Foran J, Ward SR, Lieber RL. Muscle adaptation by serial sarcomere addition 1 year after femoral lengthening. Clin Orthop Relat Res. 2007 Mar;456:250-3. doi: 10.1097/01.blo.0000246563. 58091.af.

4. Chen J, Mashouri P, Fontyn S, Valvano M, ElliottMohamed S, Noonan AM, Brown SHM, Power GA. 
The influence of training-induced sarcomerogenesis on the history dependence of force. J Exp Biol. 2020 Aug 13;223(Pt 15):jeb218776. doi: 10.1242/jeb. 218776.

5. Lucas G, Leonard TR, Carvalho A, da Silva ASR, Herzog W. Chronic uphill and downhill exercise protocols do not lead to sarcomerogenesis in mouse skeletal muscle. J Biomech. 2020 Jan 2;98:109469. doi: 10.1016/j.jbiomech.2019.109469

6. Lucas G, Leonard TR, Carvalho A, da Silva ASR, Herzog W. Chronic uphill and downhill exercise protocols do not lead to sarcomerogenesis in mouse skeletal muscle. J Biomech. 2020 Jan 2;98:109469. doi: 10.1016/j.jbiomech.2019.109469.

7. Pincheira PA, Boswell MA, Franchi MV, Delp SL, Lichtwark GA. Biceps femoris long head sarcomere and fascicle length adaptations after three weeks of eccentric exercise training. bioRxiv. January 2021:2021.01.18.427202. doi:10.1101/2021.01.18. 427202.

\#10. May 26, 2021

Extreme sarcomere shortening during drop jump in variable gravity environment

Elena Monti (1), Janice Waldvogel (2); Ramona Ritzmann (2), Kathrin Freyler (2), Kirsten Albracht $(3,4)$, Michael Helm (2), Nicola De Cesare (5), Piero Pavan (5), Carlo Reggiani (1), Albert Gollhofer (2), Marco V Narici (1)

(1) Department of Biomedical Science, University of Padova, Padova, Italy; (2) Department of Sport and Sport Science, University of Freiburg, Freiburg, Germany; (3) Institute of Biomechanics and Orthopedics, German Sport University Cologne, Cologne, Germany; (4) Department of Medical Engineering and Technomathematics, Aachen University of Applied Sciences, Aachen, Germany; (5) Department of Industrial Engineer, University of Padova, Padova, Italy

\section{* Elena Monti: elena.monti.1@phd.unipd.it}

The regulation of sarcomere length during stretchshortening cycle (SSC) activities performed in variable gravity is unknown. Drop-jumping (DJ), a common SSC activity, involves three phases: pre-activation of antigravity muscles, landing and push-off (PO) to perform a vertical jump. The ability to absorb ground impact and generate propulsive forces depends on tendon strain and fascicle length (Lf). Hence, the region of operation of sarcomeres along the muscle length-tension (L-T) relation is a determinant factor for DJ performance. This study investigated changes in Lf and sarcomere operational length throughout the phases of DJs performed in normo-gravity (1g), hyper-gravity (Hg) and hypo-gravity (hg). Fifteen volunteers performed repeated DJs in $1 \mathrm{~g}$, hg (0 to $1 \mathrm{~g}$ ), and $\mathrm{Hg}$ (1 to $2 \mathrm{~g}$ ) during a parabolic flight. Gastrocnemius medialis (GM) electromyographic (EMG) activity and Lf were measured at rest (bipedal standing), jump-off (JO, with the foot raised before jumping down), ground contact (GC), minimum ankle joint (MAJ, end of landing) and PO. GM sarcomere number ( $\mathrm{Sn}$ ) was estimated by dividing Lf measured by ultrasound at rest by sarcomere length (SL), measured in-vivo at the same joint angle $(3.16 \mu \mathrm{m})^{1}$. Changes in SL were estimated by dividing Lf in each jump phase by Sn calculated individually (Figure 1.). The sarcomere force-generating capacity was estimated from the sarcomere $\mathrm{L}-\mathrm{T}$ relation described by Walker and Schrodt. $^{2}$ At JO in 1 g, SL was $2.55 \mu \mathrm{m}$, close to the L-T relation plateau. At GC and MAJ, SL shifted to the left, down the ascending limb of the L-T curve. Fascicles and sarcomeres shortened from JO to GC (pre-activation) and behaved quasi-isometrically from GC to MAJ, enabling tendon elongation and storage of elastic energy. Surprisingly, at PO, SL was extremely short $(1.49 \mu \mathrm{m})$, a length at which only $40 \%$ of maximum sarcomere force is expected to be produced. Notably, this condition is reached with the ankle in full plantarflexion, causing maximum tendon stretch. From MAJ to PO, a marked EMG decrease seemed to confirm that most of the energy needed for the PO is provided by the tendon recoil. Lf at $\mathrm{JO}$ was shorter in hg and $\mathrm{Hg}$ than in $1 \mathrm{~g}$, reflecting a downward shift of SL along the L-T relationship (range $1.96-2.11 \mu \mathrm{m} \mathrm{Hg}$ and 2.23-2.33 $\mu \mathrm{m} \mathrm{hg).} \mathrm{During} \mathrm{pre-}$ activation, less SL shortening occurred in $\mathrm{Hg}$ and hg than in 1g, followed by lengthening of Lf and sarcomeres between GC and MAJ. Remarkably, at MAJ, Lf reached values similar to those of $1 \mathrm{~g}$, enabling sarcomeres to produce about $70 \%$ of maximum force. In the last phase, from MAJ to PO, a strong contraction markedly reduced SL to values of 1.44-1.53 $\mu \mathrm{m}$. In conclusion, regardless of the gravity level, muscle activation appears finely regulated to enable fascicles to operate in the ascending

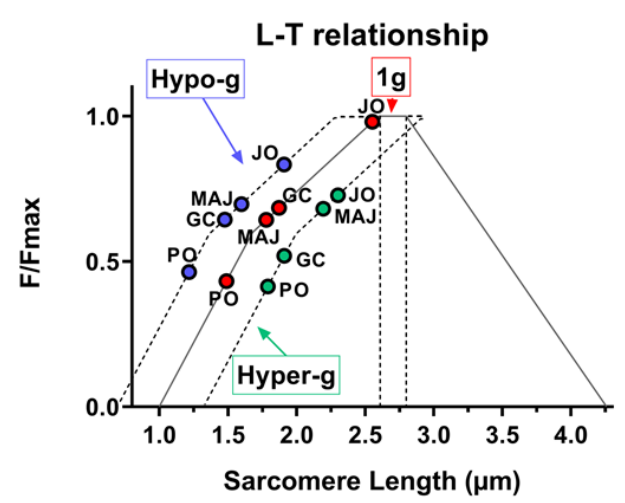

Fig 1. $S L$ in the 4 different $D J$ phases (JO, GC, $M A J, P O)$ in $1 \mathrm{~g}$ (red dots), hypo-gravity (blue dots) and hyper-gravity (green dots). The $L$ - $T$ relationship of hypo- and hypergravity has been shifted on the $x$ axis to the left and right, respectively, to allow for dots visualisation. 
portion of the L-T relation. Such neuromuscular mechanism seems to limit fascicle elongation during the breaking phase, enabling sarcomeres to reach, at MAJ, lengths similar to $1 \mathrm{~g}$. This seems a useful mechanism for anchoring the tendon, enabling storage of elastic energy and its release in the subsequent PO phase.

Keywords: sarcomere length, sarcomere operating length, drop-jump, fascicle length, muscle biomechanics, hyper-gravity, hypo-gravity

\section{References}

1. Sanchez GN, Sinha S, Liske H, Chen $X$, Nguyen V, Delp SL, Schnitzer MJ. In Vivo Imaging of Human Sarcomere Twitch Dynamics in Individual Motor Units. Neuron. 2015 Dec 16;88(6):1109-1120. doi: 10.1016/j.neuron. 2015.11.022.

2. Walker SM, Schrodt GR. I segment lengths and thin filament periods in skeletal muscle fibers of the Rhesus monkey and the human. Anat Rec. 1974 Jan;178(1):63-81. doi: 10.1002/ar.1091780107.

$$
* * * * *
$$

\#11. May 26, 2021

\section{Intraoperative and Laboratory Studies of Human Skeletal Muscle Contractures}

\section{Richard L. Lieber}

Shirley Ryan AbilityLab, Departments of Physical Medicine, Rehabilitation and Biomedical Engineering, Northwestern University, Chicago, IL, USA

* Richard L. Lieber: rlieber@sralab.org

Skeletal muscle is a highly plastic tissue, responding both to level of use and amount of neural input. After cerebral palsy (CP) altered neural input can result in muscle contractures. We have studied the mechanics and biology of muscle from children with wrist flexion contractures secondary to CP. Dramatic architectural changes are observed in these children whereby sarcomere lengths are dramatically altered relative to patients without upper motor neuron lesions. ${ }^{1}$ This suggests dramatic alterations in the regulation of muscle growth in these children. Biomechanical studies of isolated single muscle cells reveal an increased passive modulus and decreased resting sarcomere length suggesting alterations in the cellular cytoskeleton. ${ }^{2,3}$ Gene expression profiling reveals a number of "conflicting” biological pathways in spastic muscle. Specifically, this muscle adapts by altering processes related to extracellular matrix production, fiber type determination, fiber hypertrophy and myogenesis. ${ }^{4,5}$ These transcriptional adaptations are not characteristic of muscle adaptations observed in Duchenne muscular dystrophy or limb immobilization. Superimposed upon the dramatic biological and structural adaptations is a loss in the number of satellite cells that are located throughout the muscle. ${ }^{6,7}$ Even the remaining satellite cells have epigenetic changes that can dramatically influence our ability to rehabilitate these muscles. ${ }^{8}$ Recently, we showed that several anti-cancer drugs are able to reverse these epigenetic changes, thus "rescuing" the satellite cells and promoting myogenesis. Finally, based on the highly unusual transcriptional profile and neural input that these contractures have, we show that blockage of the neuromuscular junction using neurotoxins can actually assist these muscles in growing. Taken together, these results support the notion that, while contracture formation is multifactorial and neural in origin, significant structural alterations in muscle also occur. An understanding of the specific changes that occur in the muscle and extracellular matrix may facilitate the development of new conservative or surgical therapies for this devastating problem.

Keywords: satellite cell, cerebral palsy, contracture, extracellular matrix

References

1. Lieber RL, Fridén J. Spasticity causes a fundamental rearrangement of muscle-joint interaction. Muscle \& Nerve. 2002;25:265-70.

2. Lieber RL, Runesson E, Einarsson F, Fridén J. Inferior mechanical properties of spastic muscle bundles due to hypertrophic but compromised extracellular matrix material. Muscle \& Nerve. 2003;28:464-71.

3. Fridén J, Lieber RL. Spastic muscle cells are shorter and stiffer than normal cells. Muscle Nerve. 2003;27(2):157-64. Epub 2003/01/28. doi: 10.1002/mus.10247. PubMed PMID: 12548522.

4. Smith LR, Ponten E, Hedstrom Y, Ward SR, Chambers $H G$, Subramaniam S, Lieber RL. Novel transcriptional profile in wrist muscles from cerebral palsy patients. BMC Med Genomics. 2009;2:44. Epub 2009/07/16. doi: 10.1186/1755-8794-2-44. PubMed PMID: 19602279; PMCID: PMC2722667.

5. Smith LR, Chambers HG, Subramaniam S, Lieber RL. Transcriptional abnormalities of hamstring muscle contractures in children with cerebral palsy. PLoS One. 2012;7(8):e40686. doi: 10.1371/journal.pone. 0040686. PubMed PMID: 22956992; PMCID: 3431909.

6. Smith LR, Chambers HG, Lieber RL. Reduced satellite cell population may lead to contractures in children with cerebral palsy. Dev Med Child Neurol. 2013;55(3):264-70. doi: 10.1111/dmcn.12027. PubMed PMID: 23210987.

7. Dayanidhi S, Dykstra PB, Lyubasyuk V, McKay BR, Chambers HG, Lieber RL. Reduced satellite cell number in situ in muscular contractures from children with cerebral palsy. J Orthop Res. 2015;33(7):103945. doi: 10.1002/jor.22860. PubMed PMID: 25732238. 8. Domenighetti AA, Mathewson MA, Pichika R, Sibley LA, Zhao L, Chambers HG, Lieber RL. Loss of myogenic potential and fusion capacity of muscle stem cells isolated from contractured muscle in children with cerebral palsy. Am J Physiol Cell Physiol. 
2018;315(2):C247-C57. Epub 2018/04/26. doi: 10.1152/ajpcell.00351.2017. PubMed PMID: 29694232; PMCID: PMC6139501

$$
* * * * *
$$

\#12. May 26, 2021

\section{D Fiber aligned strain and strain rate measurements}

\section{Shantanu Sinha}

Dept. of Radiology, Muscle Imaging and Modeling Lab, University of California at San Diego, School of Medicine, San Diego, CA, USA

\section{* Shantanu Sinha: shsinha@ucsd.edu}

We have established velocity encoded MRI as a powerful non-invasive imaging technique to monitor skeletal muscle motion. ${ }^{1-3}$ One of the limitations of this technique is that it requires of the order of 70 consistent contractions cycles in order to image muscle motion. Recently, we implemented a 'compressed sensing' acquisition, ${ }^{4,5}$ that enables a reduction in acquisition time by factors of 4 enabling us to extend the scope of our dynamic studies: e.g., imaging multiple slices, different and higher \%MVCs and ankle positions as well accommodate senior and frail subjects. The implementation of this 'fast' technique allowed us to study muscle kinematics in 3D (3 spatial and 3 velocity directions) and at three \%MVCs in the calf muscle in young and old subjects. This latter study included diffusion tensor imaging in order to extract the fiber direction at each voxel. The principal strains and strain rates were rotated to the fiber basis at each voxel using the diffusion tensor information at each voxel. The fiber aligned strain and strain rates were not significantly different between the young and old age groups even though significant differences were found between the two age groups in the strain/ SR indices extracted in the principal basis. The Euler angles between the principal and fiber basis were calculated (represents the rotation of the strain tensor from the muscle fiber) and we compared it with the rotation angles derived from multiscale computational modeling. The multiscale modeling provides insight into the physiological mechanism responsible for the deviation of the strain from the fiber axis. Initial results indicate that the rotation of the principal strain from the fiber may arise from shear in the endomysium. The implications of these findings with respect to muscle force loss with age will be discussed.

Keywords: Aging muscle; dynamic MRI; compressed sensing; 3D strain imaging; fiber aligned strains.

\section{References}

1. Sinha S, Hodgson JA, Finni T, Lai AM, Grinstead J, Edgerton VR. Muscle kinematics during isometric contraction: Development of phase contrast and spin tag techniques to study healthy and atrophied muscles. J Magn Reson Imaging. 2004 Dec;20(6):1008-19. doi: 10.1002/jmri.20210.
2. Sinha U, Malis V, Csapo R, Moghadasi A, Kinugasa $R$, Sinha $S$. Age-related differences in strain rate tensor of the medial gastrocnemius muscle during passive plantarflexion and active isometric contraction using velocity encoded MR imaging: potential index of lateral force transmission. Magn Reson Med. 2015 May;73(5):1852-63. doi: 10.1002 /mrm.25312. Epub 2014 Jul 8.

3. Malis V, Sinha $U$, Csapo $R$, Narici $M$, Sinha $S$. Relationship of changes in strain rate indices estimated from velocity-encoded MR imaging to loss of muscle force following disuse atrophy. Magn Reson Med. 2018 Feb;79(2):912-922. doi: 10.1002/mrm.26759. Epub 2017 May 30.

4. Lustig M, Donoho D, Pauly JM. Sparse MRI: The application of compressed sensing for rapid $M R$ imaging. Magn Reson Med. 2007 Dec;58(6):118295. doi: 10.1002/mrm.21391.

5. Malis $V$, Sinha $U$, Sinha S. Compressed sensing velocity encoded phase contrast imaging: Monitoring skeletal muscle kinematics. Magn Reson Med. 2020;84:142-156. Magn Reson Med. 2020 Jul;84(1):142-156. doi: 10.1002/mrm.28100. Ерub 2019 Dec 11.

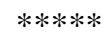

\#13. May 26, 2021

Age related structural changes in calf muscle using Quantitative Magnetic Resonance Imaging

John C White, Usha Sinha*

Department of Physics, San Diego State University, San Diego, CA, USA

*Usha Sinha: usinha@sdsu.edu

Structural and functional changes occur in skeletal muscle with age and with disuse resulting in a loss of muscle force $\mathrm{e}^{1,2}$. Contributors to the loss of muscle force with age include decrease in muscle mass, structural changes in the extracellular matrix and increase in fat and fibrotic tissue. The extracellular matrix as well as fibrotic tissue contain a relatively large fraction of macromolecules. Magnetic Resonance Imaging (MRI) provides a way to map free protons in tissue while protons bound to macromolecules (e.g., collagen) are not visible on routine MRI due to their ultra-short T2 values. Magnetization transfer contrast (MTC) enables an indirect evaluation of the macromolecular content by selective saturation of bound protons. It is hypothesized that collagen is the primary macromolecular pool responsible for the MTC effect in skeletal muscle. Collagen content in muscle increases with aging and thus, a non-invasive marker of collagen in muscle tissue has the potential to identify aging differences. Further, inflammatory changes are also known to occur in aging muscle and T1 mapping may be able to track these changes. Here, we 
measured $\mathrm{MT}_{\text {sat }}{ }^{3}$ (a semi-quantitative index of MTC) and $\mathrm{T} 1$ to explore age related changes in calf muscle. We also explored the effects of different fat suppression methods on the observed T1 values to identify the effects of incidental magnetization transfer on the measured $\mathrm{T} 1$ values. A cohort of 10 healthy young subjects ( $24.5 \pm 3$ years) and eight healthy senior subjects ( $65 \pm 8$ years) were imaged in a 3T scanner. T1 derived from the fat saturated sequence was significantly lower compared to $\mathrm{T} 1$ derived from the other three sequences (no fat suppression, water excitation -fast and normal). The decreased T1 values from the fat saturated sequence is attributed to the magnetization transfer effects of the offresonance fat saturation pulse. Significant differences were found between young and old subjects in T1 derived from the sequences with fat suppression; in all cases T1 increased with age in fat suppressed sequences ${ }^{4}$. Significant differences in $\mathrm{MT}_{\text {sat }}$ were found between the young and old cohorts with $\mathrm{MT}_{\text {sat }}$ decreasing with age. The increase in T1 values with age is hypothesized to reflect inflammatory changes in skeletal muscle and could potentially be an imaging biomarker. That T1 values from the fat unsuppressed sequence did not show age related changes is attributed to the opposing effects of increased fat infiltration (shorter T1) and increasing inflammation (longer T1), emphasizing the need for efficient fat suppression in skeletal muscle quantitative imaging. $\mathrm{MT}_{\text {sat }}$ decreased significantly with age and this decrease cannot be explained by our hypothesis of increase in collagen with age. The decrease in $\mathrm{MT}_{\text {sat }}$ may arise from decreases in muscle fibers (atrophy); the muscle fibers may themselves be the source of the macromolecular pool. In conclusion, fat suppressed T1 and $\mathrm{MT}_{\text {sat }}$ values were significantly different with age in skeletal muscle though the direction of the change in $\mathrm{MT}_{\text {sat }}$ cannot be explained due to a lack of knowledge about the macromolecular pool responsible for the MTC effect in skeletal muscle.

Keywords: Aging Muscle, macromolecular fraction content, T1 mapping

\section{References}

1. Csapo R, Malis V, Sinha U, Du J, Sinha S. Ageassociated differences in triceps surae muscle composition and strength - an MRI-based crosssectional comparison of contractile, adipose and connective tissue. BMC Musculoskelet Disord. 2014 Jun 17;15:209. doi: 10.1186/1471-2474-15-209..

2. Malis V, Sinha U, Csapo R, Narici M, Smitaman E, Sinha S. Diffusion tensor imaging and diffusion modeling: Application to monitoring changes in the medial gastrocnemius in disuse atrophy induced by unilateral limb suspension. J Magn Reson Imaging. 2019 Jun;49(6):1655-1664. doi: 10.1002/jmri.26295. Epub 2018 Dec 19.

3. Helms G, Dathe H, Kallenberg K, Dechent P. Highresolution maps of magnetization transfer with inherent correction for RF inhomogeneity and $T 1$ relaxation obtained from 3D FLASH MRI. Magn Reson Med. 2008 Dec;60(6):1396-407. doi: 10.1002/mrm.21732.

4. Marty B, Carlier PG. Physiological and pathological skeletal muscle T1 changes quantified using a fast inversion-recovery radial NMR imaging sequence. $c i$ Rep. 2019 May 2;9(1):6852. doi: 10.1038/s41598019-43398-x.

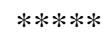

\#14. May 26, 2021

\section{Neural control of movement unraveled by electromyography}

Dario Farina,* Jaime Ibáñez

Department of Bioengineering, Imperial College London, UK

* Dario Farina: d.farina@imperial.ac.uk

The study of the interactions between the brain, spinal cord and muscles during volitional movements is key to understand the neural basis for motor control. While very important advances are being achieved regarding how brain's regions and neural populations change their activity to produce movements, we still know very little regarding how motor commands are transmitted through descending pathways to the muscles. When this problem is addressed in animal research, evoked potentials are used to study connectivity in corticospinal tracts. The limitation of this approach is that electrical stimulation of neurons provides biased information as the largest cells dominate the responses measured. Moreover, evoked potentials are not directly associated to behaviour. We have recently shown that having access to the neural code driving muscles in combination with brain signals can provide us with a unique framework to study the properties of corticomuscular transmission in terms of the expected populations of cells in the spinal cord that contribute to the neural information that reaches the muscles. This approach consists in decoding high-density EMG (HDEMG) signals into the activity of individual spinal motoneurons, ${ }^{1}$ and estimating the sources of synaptic inputs that the motoneurons receive with coherence techniques. Using this framework, we have been able to show that oscillatory activity in the brain successfully travels to the muscles using the most straightforward and fast routes available. ${ }^{2}$ This simple finding provides a strong rationale towards using simplified models of the corticospinal system to understand key questions about motor control and also regarding the mechanisms of actions of different neuromodulation techniques used in the field of neural rehabilitation. The talk will focus on the use of HDEMG for assessing the output of the spinal cord and for identifying neural connectivity between the brain and the spinal cord. These methods will be discussed in relation to their applications in neurorehabilitation technologies. 
Keywords: HDEMG, motoneuron, motor unit, neural connectivity, corticomuscular coherence.

\section{References}

1. Vecchio $A D$, Farina D. Interfacing the neural output of the spinal cord: robust and reliable longitudinal identification of motor neurons in humans. J Neural Eng. 2019 Dec 5;17(1):016003. doi: 10.1088/17412552/ab4d05.

2. Ibáñez J, A. Del Vecchio, J. C. Rothwell, S. N. Baker, D. Farina.

\section{\#15. May 26, 2021}

High Density EMG as a tool to investigate muscle in health and disease: state of the art

\section{Giuseppe De Vito}

CIR-Myo Myology Centre, Neuromuscular Physiology Laboratory, Department of Biomedical Sciences, University of Padova, Padova, Italy

* Giuseppe De Vito: giuseppe.devito@unipd.it

In recent years, the introduction of the high-density electromyography technique (HD-emg) has created new perspectives for the in vivo study of individual motor units and motor control. ${ }^{1}$ The technique which makes use of matrices containing multiple electrodes presents numerous advantages over traditional surface or needle electromyography. First it is not invasive, second it allows the study of the MU in vivo even during contractions of moderate/high intensity $(50-80 \%$ of the maximum voluntary contraction). The use of multiple electrodes matrices, combined with the possibility of recording over a broader range of contraction intensities, consents the assessment of a higher number of MUs than with the needle technique. Moreover, recent studies have shown that HD-emg allows not only to identify and characterize a specific MU but to follow it over time even after several days (weeks). ${ }^{2}$ The analysis of the MU activity in vivo, therefore, opens a window directly on the neural control of the drive directed to the muscles. Important parameters such as MU firing rate, recruitment threshold and conduction velocity can be directly assessed using the HD-emg. In particular, this technique could be applied to follow the effects of a treatment, deconditoning (disuse; bed rest studies), athletic conditioning or protein supplementation in both healthy and diseasead individuals. With aging for instance, there is denervation with loss of MUs and muscle fibers, which is partially compensated by re-innervation phenomena that can manifest themselves in the formation of large MU where previously denervated muscle fibers (usually fast twitch) are re-innervated by slow MU. ${ }^{3}$ HD-emg combined with dynamometry and posturography would allow to study the consequences of these modifications in vivo, for example during motor control tests (accuracy in maintaining muscle strength) and postural balance assessment. Defects in signal transmission between nerve endings and the muscle membrane (NMJ) are present not only in aging but also in various other pathophysiological conditions including amyotrophic lateral sclerosis $\left(\mathrm{ALS} ;{ }^{4}\right)$. Recently the HD-emg has been used to characterize the daytime variability of fasciculation firing in people suffering from ALS. ${ }^{5}$ It is obvious that this technique opens many research opportunities and therefore in my presentation.

Keywords: motor unit; high density electromyography; aging; neuromuscualr junction; recruitment threshold; firing rate.

\section{References}

1. Merletti R, Holobar A, Farina D. Analysis of motor units with high-density surface electromyography. J Electromyogr Kinesiol. 2008 Dec;18(6):879-90. doi: 10.1016/j.jelekin.2008.09.002. Epub 2008 Nov 11.

2. Del Vecchio A, Casolo A, Negro F, Scorcelletti $M$, Bazzucchi I, Enoka R, Felici F, Farina D. The increase in muscle force after 4 weeks of strength training is mediated by adaptations in motor unit recruitment and rate coding. $J$ Physiol. 2019 Apr;597(7):1873-1887. doi: 10.1113/JP277250. Epub 2019 Feb 6

3. Wu R, De Vito G, Delahunt E, Ditroilo M. Age-related Changes in Motor Function (I). Mechanical and Neuromuscular Factors. Int J Sports Med. 2020 Oct;41(11):709-719. doi: 10.1055/a-1144-3408. Еpub 2020 May 4

4. Lepore E, Casola I, Dobrowolny G, Musarò A. Neuromuscular Junction as an Entity of Nerve-Muscle Communication. Cells. 2019 Aug 16;8(8):906. doi: 10.3390/cells8080906.

5. Bashford J, Masood U, Wickham A, Iniesta R, Drakakis E, Boutelle M, Mills K, Shaw C. Fasciculations demonstrate daytime consistency in amyotrophic lateral sclerosis. Muscle Nerve. 2020 Jun;61(6):745-750. doi: 10.1002/mus.26864. Epub 2020 Apr 7.

$$
* * * * *
$$

\#16. May 26, 2021

Neuromechanical determinants of rate of force development: evidence, assumptions, and constraints

\section{Alessandro Del Vecchio}

Chair in Neuromuscular Physiology and Neural Interfacing, Department of Artificial Intelligence in Biomedical Engineering, Friedrich-Alexander University Erlangen-Nürnberg, Germany

* Alessandro Del Vecchio:

alessandro.del.vecchio@fau.de

There is a large variability in the maximal rate of force development across human individuals. It might be intuitively assumed - and it has been done for the past decades - that the muscle, with its intrinsic properties, would be the main determinant of the maximal rate of force development. Although this speculation might seem intuitively correct since the muscle represents the mechanical amplifier of the neural commands, there is 
evidence suggesting that the variability in rate of force development is mainly attributed to neural factors. ${ }^{1-3}$ Computational motor unit models and in-vivo estimates of motoneuron behavior during fast feedforward movements are indicating that it is possible to predict the rate of force development of a human individual from the neural input received by the muscle before the generation of any observable force. These studies report strong associations between rate of force development and motor unit recruitment patterns. We will discuss novel results obtained with the decomposition of high-density EMG signals during fast feedforward movements, including results obtained by measuring the spike frequency adaptation, principal component analysis applied during fast feedforward movements and also motoneuron behavior during fast kicking actions in human neonates. ${ }^{4}$

Keywords: Motor Units; Contractile Speed; Neural Drive; EMG; Decomposition.

\section{References}

1. J. E. Desmedt, E. Godaux, Ballistic contractions in fast or slow human muscles: discharge patterns of single motor units. J. Physiol. 285, 185-196 (1978).

2. M. Van Cutsem, J. Duchateau, K. Hainaut, Changes in single motor unit behaviour contribute to the increase in contraction speed after dynamic training in humans. J. Physiol. 513, 295-305 (1998).

3. A. Del Vecchio, F. Negro, A. Holobar, A. Casolo, J. P. Folland, F. Felici, D. Farina, You are as fast as your motor neurons: speed of recruitment and maximal discharge of motor neurons determine the maximal rate of force development in humans. $J$. Physiol. 597, 2445-2456 (2019).

4. A. Del Vecchio, F. Sylos-Labini, V. Mondì, P. Paolillo, Y. Ivanenko, F. Lacquaniti, D. Farina, Spinal motoneurons of the human newborn are highly synchronized during leg movements. Sci. Adv. 6, eabc3916 (2020).

\#17 May 26, 2021

HDsEMG meets neuromechanics: transferring motor unit activity into function,

\section{Francesco Negro}

\section{University of Brescia, Italy}

* Francesco Negro: francesco.negro@unibs.it

The talk will focus on the neuromechanical evaluation of human movement using high-density surface EMG (HDsEMG) technologies. After a brief discussion on the limitations of the use of surface EMG to describe neural control of movement, recent results on the decomposition of HDsEMG signals during dynamic contractions at different velocities will be presented. ${ }^{1}$ Techniques for tracking motor units across movements performed at increasing velocities will be described. ${ }^{2}$ ) As previously demonstrated in isometric contractions, ${ }^{3,4}$ the identification of the same motor units active at different speeds will show the advance in the use of HD-sEMG to investigate the neural drive to muscles in dynamic contractions. The results suggest that motor unit rate coding rather than recruitment is responsible for the control of muscle shortening and lengthening contractions at increasing velocities against a constant load. Additionally, the combination of these motor unit tracking methods with ultrasound transparent HDsEMG arrays, ${ }^{5}$ will show the real potential of closing the current gap in the study of the neuromechanics of human movement.

Keywords: dynamic contractions; HDsEMG decomposition; ultrasound.

\section{References}

1. Negro F, Muceli S, Castronovo AM, Holobar A, Farina D. Multi-channel intramuscular and surface EMG decomposition by convolutive blind source separation. J Neural Eng. 2016 Apr;13(2):026027. doi: 10.1088/1741-2560/13/2/026027.

2. Martinez-Valdes E, Negro F, Laine CM, Falla D, Mayer F, Farina D. Tracking motor units longitudinally across experimental sessions with high-density surface electromyography. J Physiol. 2017 Mar 1;595(5):1479-1496. doi: 10.1113/JP273662.

3. Del Vecchio A, Casolo A, Negro F, Scorcelletti $M$, Bazzucchi I, Enoka R, Felici F, Farina D. The increase in muscle force after 4 weeks of strength training is mediated by adaptations in motor unit recruitment and rate coding. J Physiol. 2019 Apr;597(7):1873-1887. doi: 10.1113/JP277250. Epub 2019 Feb 6.

4. Martinez-Valdes E, Farina D, Negro F, Del Vecchio A, Falla D. Early Motor Unit Conduction Velocity Changes to High-Intensity Interval Training versus Continuous Training. Med Sci Sports Exerc. 2018 Nov;50(11):2339-2350. doi: 10.1249/MSS.0000000 000001705.

5. Botter A, Beltrandi M, Cerone GL, Gazzoni M, Vieira TMM. Development and testing of acousticallymatched hydrogel-based electrodes for simultaneous EMG-ultrasound detection. Med Eng Phys. 2019 Feb;64:74-79. doi: 10.1016/j.medengphy.2018.12. 002. Epub 2018 Dec 14. 
\#18. May 26, 2021

\section{Adaptive Force - a novel concept of neuromuscular function}

Frank Bittmann*, Silas Dech, Laura V Schaefer

Department of Sports- and Health Sciences, University of Potsdam, Germany

* Frank Bittmann: bittmann@uni-potsdam.de

In motor science a specific neuromuscular function seemed to be neglected so far. The Adaptive Force (AF) reflects the capacity of adapting adequately to external forces with the intention to maintain a desired position or movement. Thereby, the external forces can be constant or varying in size. In distinction to conventional pushing isometric activity, a holding isometric action (as part of the $\mathrm{AF}$ ) requires a more complex motor control, because it has to adapt adequately to the varying impact. To stabilize a given limb position, during a continuous external force increase an ongoing forward control mechanism is necessary in order to implement anticipatory adjustments. It is hypothesized that in this process the ability of the cerebellum to predict connected motoric events in cooperation with the inferior olivary nucleus (ION) is utilized. ${ }^{1,2}$ Possibly because of its greater complexity the holding isometric function appears to be an independent neuromuscular capability. This is, inter alia, indicated by the finding that a submaximal holding isometric action ( $80 \%$ of MVIC) can only be maintained over a substantial and significant shorter time compared with the same intensity by the same muscle in a pushing manner of isometric action. ${ }^{3}$ Moreover, during measurements of AF (two different time points) the maximum holding isometric force (AFiso $_{\max }$ ) revealed substantial and significant lower amounts relatively to the MVIC (maximum pushing isometric force) of the same muscles (76.88 and 72.92\%; $\mathrm{p}=.001$ and .002 , respectively). Consequently, AFiso ${ }_{\max }$ can be discriminated from other maximal forces and should be discussed as an independent kind of muscle function. So far, there was no suitable device to detect AF. For this purpose, novel technological solutions were invented and developed. An innovative device utilizing a pneumatic principle meets the needed functionality for objective measuring of AF. During one single measurement $\mathrm{AFiso}_{\max }$ but also $\mathrm{AF}_{\max }$ can be detected. Regarding reliability of all forces between two points of time, the SEM of all torques were $1.29-5.68 \mathrm{Nm}$ and the ICCs(3.1) 0.945-0.996. AFiso max $_{\text {ax }}$ and $\mathrm{AF}_{\max }$ correlated highly $(r \geq 0.85)$. In addition, for manual examination under clinical conditions a wireless handheld device was developed. The accuracy of the device was proofed in mechanical reliability measurements without subject $(<1 \%)$. Because the force during the manual test is not generated by a technical device but a human, the course of the applied force depends on his/her skills. The ICCs(3.1) of the applied maximum force over 10 consecutive tests ranges from averagely $0.956 \pm 0.046 \pm$ in a group of beginners and to averagely $0.984 \pm 0.02$ in experts. The mean Euclidean distance between the 10 curves generated by the same person revealed a significant better reproducibility in well skilled experts compared to slightly trained examiners and also to beginners (padj $=0.047, r=0.585$ and padj $=0.015, r=$ 0.612 , respectively). ${ }^{1}$ The quick and flexible use of an objective handheld device opens up many new approaches for practice and research. A first attempt regarding the influence of different smells on the AF will be presented.

Keywords: Adaptive Force, holding isometric muscle function, motor control, olfaction

References

1. Bittmann FN, Dech S, Aehle M, Schaefer LV. Manual Muscle Testing-Force Profiles and Their Reproducibility. Diagnostics (Basel). 2020 Nov 25;10(12):996. doi: 10.3390/diagnostics10120996.

2. Lawrenson C, Bares M, Kamondi A, Kovács A, Lumb $B$, Apps R, Filip P, Manto M. The mystery of the cerebellum: clues from experimental and clinical observations. Cerebellum Ataxias. 2018 Mar 29;5:8. doi: 10.1186/s40673-018-0087-9.

3. Schaefer LV, Bittmann FN. Are there two forms of isometric muscle action? Results of the experimental study support a distinction between a holding and a pushing isometric muscle function. BMC Sports Sci Med Rehabil. 2017 May 11;9:11. doi: 10.1186/s13102-017-0075-z. $* * * * *$

\#19. May 27, 2021

High-fat diet increases the risk of environmental heatstroke in mice

Matteo Serano (1)*, Antonio Michelucci (1), Giorgia Rastelli (1), Cecilia Paolini (1), Flavia Alessandra Guarnier (2), Feliciano Protasi (1)

(1) CAST, Center for Advanced Studies and Technology; University $G$ D'Annunzio of ChietiPescara, Italy; (2) Department of General Pathology, Londrina State University, Londrina, Brazil

* Matteo Serano: matteo.serano@unich.it

Heat-stroke (HS) is a life-threatening response to heat or physical exertion characterized by an abnormal increase in body temperature $\left(>40^{\circ} \mathrm{C}\right)$ that causes dysfunction of organs, central nervous system and may end in death. ${ }^{1}$ Both exertional and environmental heat-stroke (EHS), often triggered by a hot and humid environment or by strenuous exercise performed in challenging conditions, are caused by excessive heat production in muscle, which in turn is the result of oxidative stress and abnormal $\mathrm{Ca}^{2+}$ leak from the sarcoplasmic reticulum (SR). ${ }^{2}$ As high fat diet is known to increase oxidative stress, ${ }^{3}$ the objective of the present study was to investigate the effects of high- 
fat diet in the heat-stroke susceptibility of C57bl/6 wild type (WT) mice of 4 months of age (adult). Our results show that, in comparison with mice fed with a control diet, mice after 3 months of high-fat diet dispaly: a) increased heat generation and energy expenditure (assessed by indirect calorimetry) during heat stress; b) elevated oxidative stress in both EDL and Soleus muscles; and c) enhanced sensitivity to caffeine and temperature of isolated EDL and Soleus muscles during in vitro contracture test (IVCT, the gold standard procedure to test in-vitro EHS susceptibility). Our data suggest that high-fat diet predispose mice to EHS, possibly as a result of increased oxidative stress and excessive release of $\mathrm{Ca}^{2+}$ from SR. This study may have important implications for guidelines regarding food habits during periods of intense environmental heat.

Keywords: high-fat diet, heat-stroke, oxidative stress

\section{References}

1. Bouchama A, Knochel JP. Heat stroke. N Engl J Med. 2002 Jun 20;346(25):1978-88. doi: 10.1056/NEJM ra011089

2. Durham WJ, Aracena-Parks $P$, Long C, Rossi AE, Goonasekera SA, Boncompagni S, Galvan DL, Gilman CP, Baker MR, Shirokova N, Protasi F, Dirksen R, Hamilton SL. RyR1 S-nitrosylation underlies environmental heat stroke and sudden death in Y522S RyR1 knockin mice. Cell. 2008 Apr 4;133(1):53-65. doi: 10.1016/j.cell.2008.02.042.

3. Matsuzawa-Nagata N, Takamura T, Ando $H$, Nakamura S, Kurita S, Misu H, Ota T, Yokoyama M, Honda M, Miyamoto K, Kaneko S. Increased oxidative stress precedes the onset of high-fat dietinduced insulin resistance and obesity. Metabolism. 2008 Aug;57(8):1071-7. doi: 10.1016/j.metabol 2008.03.010.

\#20. May 27, 2021

Changed patterns of muscular oscillations during a bilateral isometric motor task in Parkinson patients without tremor compared to healthy controls

\author{
Laura V Schaefer*, Silas Dech, Frank N. Bittmann
}

Regulative Physiology and Prevention, Department of Sports and Health Sciences, University of Potsdam, Germany

\section{*Laura V Schaefer: lschaefe@uni-potsdam.de}

Parkinson's disease (PD) is mostly diagnosed by clinical examinations of the cardinal symptoms rigor, bradycardia and tremor. An objective diagnostic tool in clinical routine does currently not exist. ${ }^{1}$ The motor symptoms are mainly explained by a loss of more than $50 \%$ of the dopaminergic cells in the substantia nigra. ${ }^{1-3}$ The present study is based on the hypothesis that neuromuscular parameters might show altered patterns already before that substantial degree of cell loss. Studies examining PD patients without tremor scarcely exist. The aim of the present study was to investigate the oscillatory behavior of the mechanical muscle oscillations in PD patients (PD) without tremor compared to healthy controls (Con). The mechanical muscle oscillations (MMG), force and acceleration (ACC) of 18 PD patients without tremor (in medication off state) and of 20 healthy controls (Con) were measured during a specific bilateral isometric motor task. ${ }^{4}$ The MMGs of the biceps brachii, the brachioradialis and of the pectoralis major muscles were recorded using a piezo-based measurement system. Five trials at $60 \%$ of the maximal voluntary isometric contraction (MVIC) were performed. The frequency, a specific power frequency ratio in low frequency ranges of 3 to $12 \mathrm{~Hz}$ as well as the variation and the slope of amplitude maxima were evaluated and compared between PD and Con. PD patients showed a significantly lower power frequency ratio and amplitude variation of ACC and MMGs compared to Con (power frequency ratio: $p \leq 0.001-0.004$; effect size $r=0.441-0.579$; amplitude variation: $\mathrm{p} \leq 0.001, \mathrm{r}=0.37-0.67$ ). The $95 \%$ confidence intervals were clearly disjoint. The ACC showed, furthermore, a significant difference concerning the mean frequency $(\mathrm{p}=0.009, \mathrm{r}=0.43)$. No significant differences were present concerning the slope of amplitude maxima. The power frequency ratio as well as the amplitude variation as parameters of the mechanical motor output showed clear differences between PD patients without tremor and healthy controls during this novel bilateral assessment. The bilateral setting seems to enhance the effects, which were less clear during a unilateral motor task. ${ }^{5}$ If those results will be supported by further studies an innovative biomarker for PD patients might be developed. For this propose it has to be investigated, if the results are reproducible and specific for PD. It is supposed that those mechanical muscle oscillations reflect neurodegenerative changes, which are probably especially visible during the bilateral motor task.

Keywords: Parkinson's disease, mechanomyography, bilateral motor task.

\section{References}

1. Kalia LV, Lang AE. Parkinson's disease. The Lancet. 2015;386(9996):896-912. doi:10.1016/S01406736(14) 61393-3

2. Michel PP, Hirsch EC, Hunot S. Understanding Dopaminergic Cell Death Pathways in Parkinson Disease. Neuron. 2016;90(4):675-691. doi:10.1016/j. neuron.2016.03.038

3. Poewe W, Seppi K, Tanner CM, et al. Parkinson disease. Nat Rev Dis Primers. 2017;3(1):17013. doi:10.1038/nrdp.2017.13

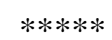


\#21. May 26, 2021

Triggered regulation of the venous capillary hemoglobin amount in superficial muscle tissue during fatiguing isometric muscle actions

Silas Dech,* Frank Bittmann, Laura V Schaefer

Division for Regulative Physiology and Prevention, Department of Sports and Health Sciences, University of Potsdam, Germany

* Silas Dech: dech@uni-potsdam.de

A high intramuscular pressure during submaximal isometric muscle actions might restrict the blood flow. ${ }^{1-5}$ Thus, the oxygenation and the relative hemoglobin amount $(\mathrm{rHb})$, which indicates the blood filling of venous capillaries, should be altered. Recently, two behavioral types were described during a fatiguing holding isometric muscle action (HIMA). ${ }^{6}$ Type I: rHb decreases and levels off into a steady state, nearly parallel to the capillaryvenous oxygen saturation (SvO2). Type II: $\mathrm{rHb}$ decreases until a reversal point (RP) and increases before leveling off into a steady state, whereby the $\mathrm{SvO} 2$ decreases further on and its steady state sets in at a lower level compared to type I. The aim of the present study was to examine an explanation of these two patterns. 12 subjects (29.75 \pm 11.14 years) performed a fatiguing HIMA with $60 \%$ of the maximal voluntary isometric contraction in a $90^{\circ}$ elbow flexion on each arm. Six subjects additionally pulled on an immovable resistance (fatiguing pulling isometric muscle action, PIMA) with the same intensity once per arm. The $\mathrm{rHb}$ and $\mathrm{SvO} 2$ were recorded by the spectrophotometric O2C-device. In type II behaviors (n $=26$ ), the RP was found at an average SvO2-level of $58.75 \pm 2.14 \%$. The SvO2 in type I behaviors $(\mathrm{n}=10)$ never reached that specific value. The level of deoxygenation could explain the two patterns. It is suggested a threshold $~ 59 \%$ triggers the increase of rHb. A possible approach regarding the compatibility of an $\mathrm{rHb}$-increase with a theoretical blood flow restriction is given.

Keywords: muscle oxygen saturation, hemoglobin amount, isometric muscle action, blood flow

\section{References}

1. Sadamoto T, Bonde-Petersen F, Suzuki Y. Skeletal muscle tension, flow, pressure, and EMG during sustained isometric contractions in humans. Eur $J$ Appl Physiol Occup Physiol 1983;51:395-408.

2. Sejersted OM, Hargens $A R$, Kardel $K R$, et al. Intramuscular fluid pressure during isometric contraction of human skeletal muscle. J Appl Physiol 1984;56:287-95.

3. Sjøgaard G, Savard G, Juel C. Muscle blood flow during isometric activity and its relation to muscle fatigue. Eur J Appl Physiol Occup Physiol 1988;57:327-35.
4. Jensen BR, Jørgensen $K$, Hargens $A R$, et al. Physiological response to submaximal isometric contractions of the paravertebral muscles. Spine 1999;24:2332-8.

5. Järvholm U, Styf $J$, Suurkula $M$, Herberts $P$. Intramuscular pressure and muscle blood flow in supraspinatus. Eur J Appl Physiol Occup Physiol 1988;8:219-24.

6. Dech S, Bittmann F, Schaefer L. Behavior of oxygen saturation and blood filling in the venous capillary system of the biceps brachii muscle during a fatiguing isometric action. Eur. J. Transl. Myol 2020 30:79-87.

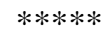

\#22. May 27, 2021

Invited Lecture: Role of iron in muscle sarcopenia

Anna Picca (1), Sunil K. Saini (2) Emanuele Marzetti (1), Christiaan Leeuwenburgh (2)*

(1) Fondazione Policlinico Universitario "Agostino Gemelli" IRCCS, Rome, Italy; (2) Department of Aging and Geriatric Research, Institute on Aging, University of Florida, USA

*Christiaan Leeuwenburgh: cleeuwen@ufl.edu

Mitochondrial dysfunction and iron $(\mathrm{Fe})$ dyshomeostasis have been identified among the mechanisms contributing to muscle aging possibly via a detrimental mitochondrialiron feed-forward loop. This loop might be partly reflected by an altered expression of mitoferrin and frataxin, larger labile iron pool and greater mitochondrial DNA damage in skeletal muscle of older adults. We quantified the labile $\mathrm{Fe}$ pool, $\mathrm{Fe}$ isotopes, and the expression of mitochondrial $\mathrm{Fe}$ handling proteins in muscle biopsies obtained from young and older adults. The expression of key proteins of mitochondrial quality control (MQC) and the abundance of the mitochondrial DNA common deletion (mtDNA4977) were also assessed. An inverse association was found between total $\mathrm{Fe}$ and $\mathrm{Fe}$ isotope ratio, indicating an increase in labile $\mathrm{Fe}$ abundance in cells with greater $\mathrm{Fe}$ content. The highest levels of labile $\mathrm{Fe}$ were detected in older participants with a Short Physical Performance Battery (SPPB) score $\leq 7$ (low-functioning, LF). Protein levels of mitoferrin and frataxin were, respectively, higher and lower in the LF group relative to young participants and older adults with SPPB scores $\geq 11$ (high-functioning, HF). The mtDNA4977 relative abundance was greater in older than in younger participants regardless of SPPB category. Higher protein levels of Pink1 were detected in LF older adults compared to young and HF older adults. Finally, the ratio between lipidated and non-lipidated LC3B, as well as p62 protein expression, was lower in older participants regardless of SPPB scores. Our findings indicate that cellular and mitochondrial $\mathrm{Fe}$ homeostasis is perturbed in the aged muscle (especially in LF older adults) as reflected by altered levels of 
mitoferrin and frataxin, which together with MQC derangements might contribute to loss of mtDNA stability.

Keywords: endurance, exercise, health, epigenetics

\section{References}

1. Picca A, Mankowski RT, Kamenov G, Anton SD, Manini TM, Buford TW, Saini SK, Calvani R, Landi $F$, Bernabei R, Marzetti E, Leeuwenburgh C. Advanced Age Is Associated with Iron Dyshomeostasis and Mitochondrial DNA Damage in Human Skeletal Muscle. Cells. 2019 Nov 27;8(12):1525. doi: 10.3390/cells8121525.

2. Picca A, Saini SK, Mankowski RT, Kamenov G, Anton SD, Manini TM, Buford TW, Wohlgemuth SE, Xiao R, Calvani R, Coelho-Júnior HJ, Landi F, Bernabei R, Hood DA, Marzetti E, Leeuwenburgh C. Altered Expression of Mitoferrin and Frataxin, Larger Labile Iron Pool and Greater Mitochondrial DNA Damage in the Skeletal Muscle of Older Adults. Cells. 2020 Dec 2;9(12):2579. doi: 10.3390/cells9122579.

3. Picca A, Mankowski $R$, Calvani R, Marzetti E, Leeuwenburgh C. Mitochondrial DNA Damage And Impaired Iron Homeostasis In Muscle Aging. Faseb Journal. 2018;32(1).

4. Hofer T, Marzetti E, Xu J, Seo AY, Gulec S, Knutson $M D$, Leeuwenburgh $C$, Dupont-Versteegden EE. Increased iron content and RNA oxidative damage in skeletal muscle with aging and disuse atrophy. Exp Gerontol. 2008 Jun;43(6):563-70. doi: 10.1016/ j.exger.2008.02.007.

5. Seo AY, Xu J, Servais S, Hofer T, Marzetti E, Wohlgemuth SE, Knutson MD, Chung HY, Leeuwenburgh C. Mitochondrial iron accumulation with age and functional consequences. Aging Cell. 2008 Oct;7(5):706-16. doi: 10.1111/j.14749726.2008.00418.x..

\#23. May 27, 2021

\section{Molecular mechanisms of cancer-induced skeletal muscle pathology}

Andy Judge,* Sarah Judge

Department of Physical Therapy, University of Florida, Gainesville FL, USA

* Andy Judge: arjudge@phhp.ufl.edu

Recent work from our lab identified that the transcription factor Forkhead boxP1 (FoxP1) is significantly increased in the skeletal muscle of tumor bearing hosts exhibiting cachexia $^{1,2}$. FoxP1 is a negative regulator of gene transcription, which has strong relevance to cancer cachexia given that gene repression is the predominant direction of change of differentially expressed genes in the skeletal muscle of cachectic cancer patients ${ }^{3}$. This talk will focus on our recently published, and unpublished, findings which demonstrate that myofiberspecific FoxP1 upregulation is sufficient to induce several features of cachexia, including loss of body weight, muscle atrophy and muscle fiber atrophy, muscle damage and expansion of the extracellular matrix, muscle weakness, and identify the gene networks associated with these outcomes; and that myofiber-specific FoxP1 is required for the normal atrophy phenotype that occurs in tumor bearing hosts.

Keywords: muscle atrophy, cachexia, FoxP1

References

1 Judge SM, Wu CL, Beharry AW, Roberts BM, Ferreira LF, Kandarian SC, Judge AR. Genome-wide identification of FoxO-dependent gene networks in skeletal muscle during C26 cancer cachexia. BMC Cancer. 2014 Dec 24;14:997. doi: 10.1186/14712407-14-997.

2 Neyroud D, Nosacka RL, Callaway CS, Trevino JG, Hu H, Judge SM, Judge AR. FoxP1 is a transcriptional repressor associated with cancer cachexia that induces skeletal muscle wasting and weakness. J Cachexia Sarcopenia Muscle. 2021 Feb 1. doi: 10.1002/jcsm.12666. Epub ahead of print.

3 Talbert EE, Cuitiño MC, Ladner KJ, Rajasekerea PV, Siebert $M$, Shakya R, Leone GW, Ostrowski MC, Paleo B, Weisleder N, Reiser PJ, Webb A, Timmers $C D$, Eiferman DS, Evans DC, Dillhoff ME, Schmidt CR, Guttridge DC. Modeling Human Cancer-induced Cachexia. Cell Rep. 2019 Aug 6;28(6):1612-1622.e4. doi: 10.1016/j.celrep.2019.07.016.

$$
* * * * *
$$

\#24. May 27, 2021

Understanding tumor-mediated muscle damage

Erin Talbert $(1,2) *$

(1) University of Iowa, Department of Health and Human Physiology, Iowa City, IA, USA; (2) University of Iowa, Holden Comprehensive Cancer Center, Iowa City, IA, USA

* Erin Talbert: erin-talbert@uiowa.edu

Cachexia is a wasting syndrome that occurs in patients with many illnesses and is characterized by pronounced loss of skeletal muscle and adipose tissue. In cancer patients, cachexia associates with increased morbidity and mortality and decreased treatment tolerance. Although advances have been made in understanding mechanisms of tumor-induced muscle loss, we lack an approved pharmacological intervention in both the United States and Europe. While animal models of cancer cachexia have suggested that activation of the ubiquitin proteasome system is crucial to muscle wasting, there is less evidence of significant increases in proteolysis in humans with cancer cachexia. ${ }^{1}$ Dysfunctional muscle regeneration has also been 
identified as a cause of muscle wasting in people and animals with cancer. ${ }^{2-4}$ Cancer appears to damage skeletal muscle, leading to activation of satellite cells. However, these muscle progenitor cells appear to be unable to fuse into and repair existing skeletal muscle fibers, leading to muscle atrophy. ${ }^{3}$ A component of this muscle damage appears to be disruption of the existing extracellular matrix surrounding skeletal muscle fibers. We propose that the tumor-induced disorganization of the extracellular matrix creates an environment that is not suitable for functional muscle regeneration, thereby contributing to muscle wasting in cancer.

Keywords: cancer cachexia, muscle damage, extracellular matrix

\section{References}

1. Talbert EE, Cuitino MC, Ladner KJ, Rajasekerea PV, Siebert M, Shakya R, et al. Modeling Human Cancerinduced Cachexia. Cell Rep. 2019 Aug 6;28(6):161222 e 4.

2. Talbert EE, Guttridge DC. Impaired regeneration: A role for the muscle microenvironment in cancer cachexia. Semin Cell Dev Biol. 2016 Jun;54:82-91.

3. He WA, Berardi E, Cardillo VM, Acharyya S, Aulino $P$, Thomas-Ahner J, et al. NF-kappaB-mediated Pax7 dysregulation in the muscle microenvironment promotes cancer cachexia. The Journal of clinical investigation. 2013 Oct 1.

4. Talbert EE, Metzger GA, He WA, Guttridge DC. Modeling human cancer cachexia in colon 26 tumorbearing adult mice. Journal of cachexia, sarcopenia and muscle. 2014 Dec;5(4):321-8.

$* * * * *$

\#25. May 27, 2021

Moderate load eccentric exercise training in
rehabilitation

\section{Hans Hoppeler}

Department of Anatomy, University of Bern, Bern, Switzerland

\section{* Hans Hoppeler: hoppeler@ana.unibe.ch}

In concentric contractions, muscles shorten and perform positive work leading to acceleration or to gain in potential energy. During eccentric contractions, activated muscles undergo lengthening and perform negative work i.e. they are used to decelerate. The physiological properties between concentric and eccentric contractions differ markedly. At similar angular velocities, the torque that muscles produce during eccentric contractions is several times higher than during concentric contractions. This is because the developed tension is remaining constant with increasing (negative) angular velocity. Muscle tissue thus suffers much higher stress during eccentric than during concentric contractions, eventually leading to muscle damage and delayed onset muscle soreness (DOMS;). ${ }^{1}$ The metabolic energy requirements are typically some four fold lower for performing a similar amount of negative (eccentric) than positive (concentric) work. ${ }^{2}$ Additionally, the same authors also showed that the integrated EMG activity for producing negative torque is only half that necessary to produce a similar amount of positive torque. This means that it is more difficult to control eccentric contractions. We have used moderate load (50-350 Watt) eccentric exercise on a recumbent eccentric ergometer to increase strength and muscle mass in subjects with coronary artery disease. ${ }^{3}$ By carefully ramping up the training loads from very low loads, delayed muscle soreness and muscle damage was circumvented. We now use controlled moderate load eccentric exercise mainly in a number of rehabilitative settings of the lower extremity. These comprise general deconditioning in particular in old age sarcopenia, reconditioning after repair of the anterior cruciate ligament as well as rehabilitation after total knee arthroplasty. Our findings are in line with studies for these conditions that show better effects of using protocols including eccentric loads. ${ }^{4,5}$ Using an eccentric recumbent ergometer allows for precise control of the training conditions. Patients work in a closed muscle chain at angular velocities similar to those experienced during normal cycling or walking. We start eccentric conditioning with 2-3 sessions per week. Each session consists of 4 bouts of 5 minutes of eccentric exercise separated by 2 minutes of rest. Initial loads are typically 25 Watt with a cadence of 40 RPM. We then increase load up to 100 Watts and cadence up to 60 RPM without provoking muscle soreness. We can prevent deconditioning with 1 training session per week.

Keywords: eccentric exercise, rehabilitation exercise training, strength

\section{References}

1. Hotfiel T, Freiwald J, Hoppe MW, Luther C, Forst R, Grim C, Bloch W, Hüttel M, Heiss R. Advances in Delayed-Onset Muscle Soreness (DOMS): Part I: Pathogenesis and Diagnostics. Sportverletz Sportschaden. 2018 Dec;32(4):243-250. doi: 10.1055/a-0753-1884.

2. Bigland-Ritchie $B$, Woods JJ. Integrated electromyogram and oxygen uptake during positive and negative work. J Physiol. 1976 Sep;260(2):26777. doi: 10.1113/jphysiol.1976.sp011515.

3. Steiner R, Meyer K, Lippuner K, Schmid H-P, Saner $H$, Hoppeler $H$. Eccentric endurance training in subjects with coronary artery disease: a novel exercise paradigm in cardiac rehabilitation? Eur $J$ Appl Physiol. 2004 May;91(5-6):572-8. doi: 10.1007/s00421-003-1000-6. 
\#26. May 27, 2021

SRF-mediated mechanotransduction is essential for the response to exercise in cancer patients and animal models

Medhi Hassani (1,2,3), Alexandra Baccam (1,2,3), Alexandra Benoni (1,2,3), Caterina Gargano (2,4), Gabriela Salim de Castro (5), Joana Alves (5), Sara Chiappalupi (3,6), Viviana Moresi (1,3), Sergio Adamo (1,3), Francesca Riuzzi (3,6,), Guglielmo Sorci (3,6,), Maurizio Muscaritoli (7), Marilia Seelaender $(5,8)$, Athanassia Sotiropulos (9), Zhigang Xue (2), Zhenlin Li (2), Onnik Agbulut (2), Dario Coletti $(1,2,3)^{*}$

(1) Dept.of Anatomical, Histological, Forensic and Orthopedic Sciences, Sapienza University of Rome, Rome, Italy; (2) CNRS UMR 8256, INSERM ERL U1164, Biological Adaptation and Aging B2A, Sorbonne Université, Paris, France; (3) Interuniversity Institute of Myology, Perugia, Italy; (4) Dept. of Life Sciences, University of Modena e Reggio Emilia, Modena, Italy; (5) Dept. of Cell and Tissue Biology, University of São Paulo, SP, Brazil; (6) Dept. of Medicine and Surgery, University of Perugia, Perugia, Italy; (7) Dept.of Clinical Medicine, Sapienza University of Rome, Rome, Italy; (8) Dept.of Surgery, Faculdade de Medicina, LIM26 HC, University of São Paulo, SP, Brazil; (9) INSERM U1016, Institut Cochin, Paris, France

* Dario Coletti: dario.coletti@uniroma1.it

Exercise training is used as an anti-cachexia treatment in clinical trials, due to its capacity to regulate both the metabolic disturbances and the severe muscle wasting which characterize cachexia ${ }^{1,2}$. Most of the molecular mechanisms underlying exercise beneficial effects are still unknown. Serum Response Factor (SRF) is a transcription factor of pivotal importance for muscle homeostasis, acting as a mechanosensor depending on muscle contraction ${ }^{3}$. We show that cancer patients have reduced SRF activity associated to cachexia, but exercise increases SRF expression even in this condition, likely favoring the rescue of SRF transcriptional activity. To address this issue, we exploited a pre-clinical model of cancer cachexia (C26-bearing mice), with a subset of the mice exercised by wheel running. We found that exercise rescues SRF expression in a dose-dependent manner, while also counteracting cachexia. Furthermore, exercise has no longer effect in SRF-KO mice showing that SRF is necessary for exercise effectiveness. SRF transcriptional activity promotes the expression of several target genes, including pro-myogenic factors as well as IL-6 and IL-4. The latter is sufficient to counteract the negative effects of tumor-derived factors on muscle cells ${ }^{4}$. We also show that exercise promotes the downregulation of Pax7 and, thus, the incorporation of nuclei in muscle fibers. Finally, we show in vitro that the purely mechanical stimulation of myotubes mimics exercise (including SRF activation, IL-4 secretion and myoblast incorporation into myotubes) being sufficient to counteract the negative effects of tumor-derived factors. Our findings highlight a model whereby the mechanical stimulation of the musculature, through the essential activation of the SRF-dependent pathway, has paracrine/endocrine effects, consisting of IL-6 and IL-4 secretion by the muscle itself. These two cytokines have multiple, positive effects on both the muscle fibers (or myotubes, in vitro) and the activated myoblasts: indeed, protein degradation is diminished in the muscle fibers, whilst myoblast are recruited to the muscle fibers contributing to maintain muscle homeostasis in the presence of cachectic factors. We propose the importance of the mechanical stimulation, induced by exercise or other means, for the regulation of muscle homeostasis and we propose that IL-4 treatment may mimic the beneficial effects of exercise.

Keywords: muscle wasting, cancer cachexia, SRF, exercise, mechanotransduction

\section{References}

1. De Castro GS, Simoes E, Lima JDCC, Ortiz-Silva M, Festuccia WT, Tokeshi F, Alcântara PS, Otoch JP, Coletti D, Seelaender M. Human Cachexia Induces Changes in Mitochondria, Autophagy and Apoptosis in the Skeletal Muscle. Cancers (Basel). 2019 Aug 28;11(9):1264.

2 Berardi E, Madaro L, Lozanoska-Ochser B, Adamo $S$, Thorrez L, Bouche M, Coletti D. A Pound of Flesh: What Cachexia Is and What It Is Not. Diagnostics (Basel). 2021 Jan 12;11(1):116.

3 Djemai H, Hassani M, Daou N, Li Z, Sotiropoulos A, Noirez P, Coletti D. Srf KO and wild-type mice similarly adapt to endurance exercise.Eur J Transl Myol. 2019 Jun 7;29(2):8205.

4 Ratio. Baccam A, Benoni-Sviercovich A, Rocchi M, Moresi V, Seelaender M, Li Z, Adamo S, Xue Z, Coletti D. The Mechanical Stimulation of Myotubes Counteracts the Effects of Tumor-Derived Factors Through the Modulation of the Activin/Follistatin Front Physiol. 2019 Apr 24;10:401

$$
* * * * *
$$

\#27. May 27, 2021

Ghrelin in Cancer Cachexia and Aging-Related Sarcopenia

\section{Jose M. Garcia}

University of Washington School of Medicine and GRECC Puget Sound VA HCS, Seattle, USA

* Jose M. Garcia: Jg77@uw.edu

Muscle mass and function loss are key components of both cancer cachexia and age-related sarcopenia. Other features such as changes in food intake, energy expenditure, inflammation and fat mass and metabolism 
are differentially modulated in these syndromes. In fact, the co-occurrence of obesity in age-related sarcopenia worsens its prognosis; whereas, higher body weight is known to have a protective effect in cancer-related cachexia. Ghrelin is a potent growth hormone (GH) secretagogue known to increase muscle and fat by increasing food intake and possibly by decreasing inflammation and energy expenditure. ${ }^{1}$ Hence, activation of the ghrelin receptor GHSR1a by ghrelin or ghrelin receptor agonists has been proposed as a potential therapeutic target for cancer-related cachexia, ${ }^{2-3}$ and agerelated sarcopenia. ${ }^{4}$ This talk will focus on the differential effects and molecular mechanisms of action of ghrelin in rodent models of sarcopenic obesity and tumor-induced cachexia, and the potential therapeutic implications of these recent research findings.

Keywords: GHSR-1a, muscle wasting; tumor; growth hormone secretagogues.

\section{References}

1. Garcia JM, Boccia RV, Graham CD, Yan Y, Duus EM, Allen S, Friend J. Anamorelin for patients with cancer cachexia: an integrated analysis of two phase 2, randomised, placebo-controlled, double-blind trials. Lancet Oncol. 2015 Jan;16(1):108-16. doi: 10.1016/S1470-2045(14)71154-4. Epub 2014 Dec 16. PubMed PMID: 25524795.

2. Chen JA, Splenser A, Guillory B, Luo J, Mendiratta $M$, Belinova B, Halder T, Zhang G, Li YP, Garcia JM. Ghrelin prevents tumour- and cisplatin-induced muscle wasting: characterization of multiple mechanisms involved. J Cachexia Sarcopenia Muscle. 2015 Jun;6(2):132-43. doi: 10.1002/jcsm.12023. Epub 2015 Apr 22. PubMed PMID: 26136189; PubMed Central PMCID: PMC4458079.

3. Liu H, Luo J, Guillory B, Chen JA, Zang P, Yoeli JK, Hernandez Y, Lee II, Anderson B, Storie M, Tewnion A, Garcia JM. Ghrelin ameliorates tumor-induced adipose tissue atrophy and inflammation via Ghrelin receptor-dependent and -independent pathways. Oncotarget. 2020 Sep 1;11(35):3286-3302. doi: 10.18632/oncotarget.27705. eCollection 2020 Sep 1. PubMed PMID: 32934774; PubMed Central PMCID: PMC7476735.

4. Guillory B, Chen JA, Patel S, Luo J, Splenser A, Mody A, Ding $M$, Baghaie S, Anderson B, Iankova B, Halder T, Hernandez Y, Garcia JM. Deletion of ghrelin prevents aging-associated obesity and muscle dysfunction without affecting longevity. Aging Cell. 2017 Aug;16(4):859-869. doi: 10.1111/acel.12618. Epub 2017 Jun 6. PubMed PMID: 28585250; PubMed Central PMCID: PMC5506439
\#28. May 27, 2021

Exercise and chemotherapy-related muscle weakness

\section{Ashley J. Smuder}

Department of Applied Physiology and Kinesiology, University of Florida, Gainesville, FL USA

* Ashley J. Smuder: asmuder@ufl.edu

Chemotherapy diminishes strength and promotes muscular fatigue in cancer patients. Musculoskeletal complications of cancer chemotherapy include lowerextremity weakness, dyspnea, loss of functional performance and muscle wasting. Growing evidence supports the notion that participation in regular bouts of physical activity improves muscular strength, reduces fatigue and enhances quality of life in cancer survivors. However, while exercise training in patients receiving chemotherapy may mitigate the negative consequences of cancer treatment on physical function, debate remains regarding the optimal exercise prescription for patients and complications such as age, co-morbidities and treatment related side effects limit its use. Alternatively, exercise is an effective tool that can be used to develop therapeutic countermeasures designed to reproduce the beneficial effects of increased muscular activity. In a preclinical model of anthracycline myotoxicity, we have shown that exercise training in combination with doxorubicin reduces chemotherapy-associated weakness and atrophy in both respiratory and hind limb muscles. Our data suggests that exercise training can improve mitochondrial iron homeostasis, resulting in a reduction in mitochondrial reactive oxygen species production and preservation of mitochondrial respiratory capacity when performed prior to doxorubicin treatment. Further, this study identifies novel exercise-induced adaptations to skeletal muscle protein transporters with the potential to alter doxorubicin muscular localization and iron homeostasis.

Keywords: doxorubicin; diaphragm; exercise.

\section{References}

1. Smuder AJ. Exercise stimulates beneficial adaptations to diminish doxorubicin-induced cellular toxicity. Am J Physiol Regul Integr Comp Physiol. 2019 Nov 1 1;317(5):R662-R672. PMID: 31461307.

2. Morton AB, Huertas AM, Hinkley JM, IchinosekiSekine N, Christou DD and Smuder AJ. Mitochondrial accumulation of doxorubicin in cardiac and diaphragm muscle following exercise preconditioning. Mitochondrion. 2019 Mar;45:52-62. PMID: 29474837. 
\#29. May 27, 2021

\section{Mitochondrial Permeability Transition as Novel Mechanism of Skeletal Muscle Atrophy}

Sarah K. Burke (1,3), Trace Thome (2), Liam Fitzgerald (1), Terence Ryan (2), Russell T. Hepple (1)*

(1) Department of Physical Therapy, and Department of Physiology \& Functional Genomics, University of Florida, USA; (2) Department of Applied Physiology \& Kinesiology, University of Florida, USA; (3) Rehabilitation Sciences Doctoral Program, University of Florida, USA

\section{* Russell T. Hepple: rthepple@ufl.edu}

Mitochondria are implicated in atrophy of aging skeletal muscle, yet the link between specific changes in mitochondrial function and muscle atrophy has not been established. Prior studies have identified key roles for mitochondrial reactive oxygen species (ROS) and activation of caspase 3 in driving atrogene expression and proteolytic cleavage of actomyosin, respectively. Notably, mitochondrial permeability transition (MPT), an event which involves the formation of a non-specific pore across the mitochondrial inner membrane, results in a large burst of ROS and release of a variety of mitochondrial proteins, including cytochrome c which activates caspase 3. Despite this, the role of MPT as an upstream event driving mitochondrial ROS- and caspase 3-mediated atrophy signaling has not been considered. To address this gap, in a muscle cell culture system, induction of MPT using doxorubicin (Dox) was coincident with AIF translocation to myotube nuclei and a large burst in ROS. Furthermore, Dox-induced MPT caused muscle atrophy that was blocked using inhibitors of MPT (cyclosporin A, bonkrekic acid, isoxazole 63), inhibiting mitochondrial ROS (using MitoTempo), or knocking down caspase 3 (using shRNA). Furthermore, maintaining single mouse flexor digitorum brevis (FDB) muscle fibers in culture caused muscle fiber atrophy that was blocked by the MPT inhibition. Together, these results identify MPT as a novel mechanism of skeletal muscle atrophy that depends upon both mitochondrial ROS and caspase 3 .

Keywords: Mitochondria, mitochondrial permeability transition pore, reactive oxygen species, caspase 3

\section{References}

1. Hyatt H, Deminice R, Yoshihara T, Powers SK. Mitochondrial dysfunction induces muscle atrophy during prolonged inactivity: A review of the causes and effects. Arch Biochem Biophys, 2019;662, 49-60. doi:10.1016/j.abb.2018.11.005

2. Larsson L, Degens $H$, Li M, Salviati L, Lee YI, Thompson W, Kirkland JL, Sandri M. Sarcopenia: Aging-Related Loss of Muscle Mass and Function. Physiol Rev. 2019 Jan 1;99(1):427-511. doi: 10.1152/physrev.00061.2017
3. Marzetti E, Hwang JC, Lees HA, Wohlgemuth SE, Dupont-Versteegden EE, Carter CS, Bernabei R, Leeuwenburgh C. Mitochondrial death effectors: relevance to sarcopenia and disuse muscle atrophy. Biochim Biophys Acta. 2010 Mar;1800(3):235-44. doi: 10.1016/j.bbagen.2009.05.007. Epub 2009 May 18.

4. Workeneh BT, Rondon-Berrios H, Zhang L, Hu Z, Ayehu G, Ferrando A, Kopple JD, Wang H, Storer T, Fournier M, Lee SW, Du J, Mitch WE. Development of a diagnostic method for detecting increased muscle protein degradation in patients with catabolic conditions. J Am Soc Nephrol. 2006 Nov;17(11):3233-9. doi: 10.1681/ASN.2006020131. Epub 2006 Sep 27.

\#30. May 27, 2021

Skeletal muscle adaptations to cancer and chemotherapeutics in patients and the protective effects of exercise

\section{Michael J. Toth}

\section{Department of Medicine, University of Vermont,} Burlington, USA

* Michael J. Toth: michael.toth@med.uvm.edu

Cancer and its treatment can profoundly affect skeletal muscle, causing atrophy, impaired contractility and mitochondrial dysfunction. These maladaptations are best characterized in late-stage patients, where tumorand treatment-related factors likely contribute to muscle deficits. Muscle atrophy and dysfunction, however, are observed in patients with early-stage disease, who comprise a large proportion of the cancer survivor population and will bear the burdens of muscle atrophy and dysfunction throughout their disease-free lifetime. Cancer treatments represent a likely mediator of skeletal muscle maladaptations in early-stage patients and considerable evidence has accumulated from pre-clinical work showing how a variety of chemotherapeutics promote muscle atrophy and dysfunction. At present, no therapeutics that specifically target skeletal muscle maladaptations with cancer and its treatment have been proven effective. Physical exercise, however, has been shown to mitigate numerous symptoms and side effects of cancer and its treatment and has been proven safe. This presentation will review skeletal muscle phenotypes in human cancer patients receiving chemotherapy with and without tumor burden, potential mechanisms underlying the effects of chemotherapeutics on skeletal muscle and the potential protective effects of exercise.

Keywords: cancer; chemotherapy; exercise; atrophy.

\section{References}

1. Mijwel S, Cardinale DA, Norrbom J, Chapman M, Ivarsson $N$, Wengström $Y$, Sundberg CJ, Rundqvist $H$. 
Exercise training during chemotherapy preserves skeletal muscle fiber area, capillarization, and mitochondrial content in patients with breast cancer. FASEB J. 2018 Oct;32(10):5495-5505. doi: 10.1096/fj.201700968R. Epub 2018 May 11

2. Guigni BA, Fix DK, Bivona JJ 3rd, Palmer BM, Carson JA, Toth MJ. Electrical stimulation prevents doxorubicin-induced atrophy and mitochondrial loss in cultured myotubes. Am J Physiol Cell Physiol. 2019 Dec 1;317(6):C1213-C1228. doi: 10.1152/ ajpcell.00148.2019. Epub 2019 Sep 18.

3. Toth MJ, Voigt TB, Tourville TW, Prior SM, Guigni BA, Schlosberg AV, Smith IB, Forest TJ, Kaufman PA, Wood ME, Rehman H, Dittus K. Effect of neuromuscular electrical stimulation on skeletal muscle size and function in patients with breast cancer receiving chemotherapy. J Appl Physiol (1985). 2020 Jun 1;128(6):1654-1665. doi: 10.1152/japplphysiol.00203.2020. Epub 2020 May 7.

\section{$* * * * *$}

\#31. May 27, 2021

\section{Physiological networks of training outcomes in elderly}

Danilo Bondi (1)*, Vittore Verratti (2), Stefania Fulle (1), Tiziana Pietrangelo (1)

(1) Department of Neuroscience, Imaging and Clinical Sciences, University "G'd'Annunzio" of Chieti Pescara, Italy; (2) Department of Psychological, Health and Territorial Sciences, University "G'd'Annunzio" of Chieti - Pescara, Italy

\section{* Danilo Bondi: danilo.bondi@unich.it}

A steady decline in the variety of the interconnected physiological patterns occurs throughout ageing. Network science approaches in ageing research represent a recent advancement of the field, with interesting perspectives both for predict adverse outcomes and evaluate interventions. To provide a piece of evidence, we analyzed a dataset obtained from the project named “A.M.A. la terza età” (2007-2019), consisting herein of 24 participants (11 F and $13 \mathrm{M}, 71.2 \pm 4.2$ years) who performed endurance training (ET) and 45 (15 F and 32 M, $69.6 \pm 4.2$ years) who were trained with neuromuscular electrical stimulation (NMES). Four networks were constructed resulting from data the two protocols, before and after a 12-week training period. The variables as nodes consisted of isometric strength of quadriceps, handgrip strength, five times sit-to-stand test, timed up-and-go test, body mass index, and estimated fat mass. Edges consisted of partial correlation coefficients. Graphical lasso regularization technique and extended Bayesian information criterion $(\gamma=0.25)$ were implemented. The stability of edge-weights and the bootstrapped significant difference of centrality measures were evaluated; density of edges, Jaccard Similarity Index (JSI), and weighted JSI were computed to compare the networks. Among the results, in both protocols a reduction in network density after the training period occurred. The ET group underwent a more marked alteration of physiological interconnections after the training period than NMES group. We suggest strength may be considered the most accurate centrality measure in case of low sample size, and comparisons of weighted networks should be conducted by evaluating different metrics, i.e., local, global, weight-related, sign-related. The disentanglement of motor performances and metabolic indexes should represent a threat to be combated. A cross-sectional baseline evaluation of networked parameters may add novel insights for defining group homogeneity. The effectiveness of physical exercise may be evaluated in terms of local and global properties of physiological networks, ranking the variables in terms of their centrality, computing emergent properties of graphs, and applying adequate network comparison methods.

Keywords: network physiology, ageing research, physical exercise

\section{References}

1. Sturmberg JP, Bennett JM, Picard M, Seely AJE. The trajectory of life. Decreasing physiological network complexity through changing fractal patterns. Front Physiol. 2015;6. doi:10.3389/fphys.2015.00169

2. Bondi D, Jandova T, Verratti V, Pietrangelo T. A.M.A. la terza età: dalla valutazione integrata all'intervento multi-dominio per un invecchiamento sano. $V$ Convegno Nazionale dell'Associazione Italiana per gli Studi sulla Qualità della Vita; Genova University Press; 2018:33-39.

3. Epskamp S, Borsboom D, Fried EI. Estimating psychological networks and their accuracy: A tutorial paper. Behav Res Methods. 2018;50(1):195212. doi:10.3758/s13428-017-0862-1

$$
* * * * *
$$

\#32. May 27, 2021

Novel mechanisms of uremic myopathy in chronic kidney disease

\section{Terence E. Ryan}

University of Florida, Gainesville, FL, USA

\section{* Terence E. Ryan: ryant@ufl.edu}

It is well known that chronic kidney disease (CKD) leads to a progressive and debilitating myopathy characterized by muscle weakness, fatigue, and atrophy/wasting that negatively impact functional independence and quality of life. Over the past several decades, numerous catabolic pathways have been identified as being upregulated in CKD contributing to the loss of muscle mass and function. Given the diversity of these pathways, a major gap in knowledge stems from our limited understanding of the systemic events that stimulate these catabolic 
pathways in CKD, and thus there are no readily available treatments for successfully preventing myopathy in CKD. To this end, impaired renal function results in elevation of many uremic toxins that negatively impact muscle. Several established uremic toxins are derived from tryptophan catabolism via kynurenine and indole pathways. The most widely studied of the tryptophan metabolites, indoxyl sulfate and kynurenines, causes muscle atrophy and mitochondrial dysfunction in mice with normal kidney function, although the underlying mechanisms are unknown. This presentation will focus on emerging data demonstrating molecular pathways that link uremic toxins to skeletal muscle pathology, including activation of proteolytic pathways and mitochondrial dysfunction.

Keywords: Novel mechanisms; uremic myopathy; chronic kidney disease.

\section{References}

1. Khattri RB, Thome T, Ryan TE. Tissue-Specific $1 H$ NMR Metabolomic Profiling in Mice with AdenineInduced Chronic Kidney Disease. Metabolites. 2021 Jan 10;11(1):45. doi: 10.3390/metabo11010045.

2. Thome T, Kumar RA, Burke SK, Khattri RB, Salyers ZR, Kelley RC, Coleman MD, Christou DD, Hepple $R T$, Scali ST, Ferreira LF, Ryan TE. Impaired muscle mitochondrial energetics is associated with uremic metabolite accumulation in chronic kidney disease. JCI Insight. 2021;6(1):e139826. 2021. doi: 10.1172/ jci.insight.139826.

3. Thome T, Salyers ZR, Kumar RA, Hahn D, Berru FN, Ferreira LF, Scali ST, Ryan TE. Uremic metabolites impair skeletal muscle mitochondrial energetics through disruption of the electron transport system and matrix dehydrogenase activity. Am J Physiol Cell Physiol. 2019 Oct 1;317(4):C701-C713. doi: 10.1152/ajpcell.00098.2019. Epub 2019 Jul 10.

$$
* * * * *
$$

\#33. May 27, 2021

\section{Sarcopenia and loss of muscle quality}

\section{Marco Narici}

CIR-Myo Myology Centre, Neuromuscular Physiology Laboratory, Department of Biomedical Sciences, University of Padova, Italy

\section{* Marco Narici: marco.narici@unipd.it}

Sarcopenia, the age-related loss of muscle mass and strength, affects \&gt;50\% of the population aged $75 \mathrm{yr}$ and over and is a main cause of impaired physical performance and reduced mobility. ${ }^{1}$ Amongst the several factors contributing to sarcopenia neurodegenerative changes are regarded as primary drivers of this condition, ${ }^{2}$ and responsible for $\alpha$-motoneurons and neuromuscular junction (NMJ) degeneration, for muscle fibre denervation, ${ }^{2}$ which, also fuelled by mitochondrial dysfunction and oxidative damage, ${ }^{3}$ lead to loss of motor units and muscle weakness. One of the major functional characteristics of sarcopenia is the disproportionate decrease of muscle strength, known as loss of 'muscle quality': at the age of 80 yrs, the loss of muscle strength is about 4-fold greater than that of muscle size. ${ }^{4}$ This intrinsic muscle weakness, also known as a deterioration in 'muscle quality' has traditionally been attributed to a decrease in fibre specific tension, reduced excitationcontraction coupling, reduced neural drive, accumulation of non-contractile tissue, ${ }^{2}$ and changes in muscle architecture and tendon properties. ${ }^{5}$ However, new evidence suggests that this disproportionate loss of force may also arises from changes in the extracellular matrix (ECM) and of associated proteins, leading to a decrease in lateral force transmission, ${ }^{6}$ which in young muscle normally contributes to $>50 \%$ of muscle force output, ${ }^{7}$ and more that 3 -fold of the force measured in fibre bundles as opposed to the same number of fibres deprived of ECM. ${ }^{8}$ Indeed, in rat muscle, lateral force transmission is markedly reduced in old animals (-20\%) and even more in very old ones (-44\%). ${ }^{9}$ This suggests that muscle weakness associated with sarcopenia may not only originate from muscular changes but also from alterations affecting the ECM leading to a decrease in lateral force transmission. Recent work suggests that estimations of lateral force transmission in humans may be obtained from shear strain measured in vivo from MRI strain tensor during graded muscle contractions. ${ }^{10}$ This experimental approach seems particularly promising since fibre shear during contraction is assumed to be related to lateral force transmission. ${ }^{11}$

Keywords: Muscle strength; aging; muscle quality tissue; skeletal muscle.

\section{References}

1. Narici MV, Maffulli N. Sarcopenia: characteristics, mechanisms and functional significance. $\mathrm{Br}$ Med Bull. 2010;95:139-59. doi: 10.1093/bmb/ldq008. Epub 2010 Mar 2.

2. Gonzalez-Freire $M$, de Cabo $R$, Studenski SA, Ferrucci L. The Neuromuscular Junction: Aging at the Crossroad between Nerves and Muscle. Front Aging Neurosci. 2014 Aug 11;6:208. doi: 10.3389/fnagi.2014.00208.

3. Gouspillou G, Picard M, Godin R, Burelle Y, Hepple RT. Role of peroxisome proliferatoractivated receptor gamma coactivator 1-alpha (PGC-1 $\alpha$ ) in denervation-induced atrophy in aged muscle: facts and hypotheses. Longev Healthspan. 2013 Aug 1;2(1):13. doi: 10.1186/2046-2395-2-13.

4. Moore AZ, Caturegli G, Metter EJ, Makrogiannis $S$, Resnick SM, Harris TB, Ferrucci L. Difference in muscle quality over the adult life span and biological correlates in the Baltimore Longitudinal Study of Aging. J Am Geriatr Soc. 2014 Feb;62(2):230-6. doi: 10.1111/jgs.12653. 
5. Reeves ND, Narici MV, Maganaris CN. Myotendinous plasticity to ageing and resistance exercise in humans. Exp Physiol. 2006 May;91(3):483-98. doi: 10.1113/expphysiol.2005.032896. Epub 2006 Feb 9.

6. Kragstrup TW, Kjaer M, Mackey AL. Structural, biochemical, cellular, and functional changes in skeletal muscle extracellular matrix with aging. Scand J Med Sci Sports. 2011 Dec;21(6):749-57. doi: 10.1111/j.1600-0838.2011.01377.x. Еpub 2011 Aug 18.

7. Huijing PA, Baan GC, Rebel GT. Nonmyotendinous force transmission in rat extensor digitorum longus muscle. J Exp Biol. 1998 Mar;201(Pt 5):683-91.

8. Meyer GA, Lieber RL. Elucidation of extracellular matrix mechanics from muscle fibers and fiber bundles. J Biomech. 2011 Feb 24;44(4):771-3. doi: 10.1016/j.jbiomech.2010.10.044. Epub 2010 Nov 18.

9. Ramaswamy KS, Palmer ML, van der Meulen JH, Renoux A, Kostrominova TY, Michele DE, Faulkner JA. Lateral transmission of force is impaired in skeletal muscles of dystrophic mice and very old rats. J Physiol. 2011 Mar 1;589(Pt 5):1195-208. doi: 10.1113/jphysiol.2010.201921.

10. Sinha U, Malis V, Chen JS, Csapo R, Kinugasa R, Narici MV, Sinha S. Role of the Extracellular Matrix in Loss of Muscle Force With Age and Unloading Using Magnetic Resonance Imaging, Biochemical Analysis, and Computational Models. Front Physiol. 2020 Jun 18;11:626. doi: 10.3389/fphys.2020.00626.

11. Sharafi B, Blemker SS. A mathematical model of force transmission from intrafascicularly terminating muscle fibers. J Biomech. 2011 Jul 28;44(11):2031-9. doi: 10.1016/j.jbiomech.2011. 04.038. Epub 2011 Jun 14.

$* * * * *$

\#34. May 27, 2021

Exploring the TGF $\beta$ network to identify novel regulators of skeletal muscle adaptation

\section{Paul Gregorevic}

\section{The University of Melbourne, Australia}

* Paul Gregorevic: pgre@unimelb.edu.au

The Transforming Growth Factor- $\beta$ (TGF $\beta$ ) signaling network is an important regulator of skeletal muscle mass and function and, thus, is an attractive therapeutic target for muscle disease and sarcopenia. We have observed that follistatin-based interventions (which modulate TGF- $\beta$ network activity) can promote muscle hypertrophy that ameliorates aging-associated muscle wasting. However, the muscles of old sarcopenic mice demonstrate reduced response to follistatin compared with the muscles of young-adult mice. Using quantitative transcriptomic and proteomic methods to examine the mechanisms by which the TGF $\beta$ network regulates muscle adaptation, we identified that the E3 ubiquitin ligase, ankyrin repeat and SOCS box protein 2 (ASB2) is reduced in young-adult muscles in response to follistatin treatment, but not in similarly-treated old sarcopenic muscles. Preventing repression of ASB2 in young-adult muscles diminished follistatin-induced muscle hypertrophy. Furthermore, ASB2 overexpression in mouse muscles caused muscle atrophy and impairment of force-producing capacity, while ASB2 knockdown induced muscle hypertrophy, thereby demonstrating that ASB2 is a TGF $\beta$ network-responsive negative regulator of muscle mass. Using mass spectrometry to investigate ASB2-mediated changes to the muscle proteome, we found that ASB2-induced muscle atrophy and dysfunction were associated with the downregulation of mitochondrial and contractile protein abundance, and the upregulation of proteins involved in proteasomemediated proteolysis, protein synthesis, and the cytoskeleton/sarcomere. Increased ASB2 also resulted in altered proteome ubiquitination, however, there was no simple relationship between changes in ubiquitination status and protein abundance. Using label-free proteomics to identify proteins interacting with wild-type ASB2 and a mutant ASB2 lacking the C-terminal SOCS box domain (dSOCS), we found that ASB2 interacted with a range of cytoskeletal and nuclear proteins. Combined, the findings provide insight into the transcription and translation events governing follistatinmediated skeletal muscle adaptation, identify ASB2 as a regulator of muscle mass implicated in the potential adaptive dysfunction of old muscles, and highlight the complex proteome and ubiquitinome changes that occur during ASB2-mediated muscle atrophy and dysfunction.

Keywords: TGF $\beta$, Follistatin, muscle hypertrophy, transcriptomics, ASB2, E3 ubiquitin ligase, muscle atrophy, proteomics

\section{References}

1. Goodman CA, Davey JR, Hagg A, Parker BL, Gregorevic P. Dynamic Changes to the Skeletal Muscle Proteome and Ubiquitinome Induced by the E3 Ligase, ASB2 $\beta$. Mol Cell Proteomics. 2021 Jan 28:100050. doi: 10.1016/j.mcpro.2021.100050. Epub ahead of print.

2. La M, Lu L, Lovrecz G, Hagg A, Colgan TD, Qian H, Gregorevic P, Harrison CA. Activin A-Induced Cachectic Wasting Is Attenuated by Systemic Delivery of Its Cognate Propeptide in Male Mice. Endocrinology. 2019 Oct 1;160(10):2417-2426. doi: 10.1210/en.2019-00257.

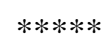


\#35. May 27, 2021

Invited Lecture: Calcium Entry Units: focus on the alternative door for calcium ions in skeletal muscle

\section{Feliciano Protasi}

\section{CAST, Center for Advanced Studies and Technology; University G D'Annunzio of Chieti-Pescara, Italy}

* Feliciano Protasi: feliciano.protasi@unich.it

Store-operated $\mathrm{Ca}^{2+}$ entry (SOCE) is a ubiquitous cellular $\mathrm{Ca}^{2+}$ influx mechanism, first described in non-excitable cells, that is triggered by depletion of intracellular $\mathrm{Ca}^{2+}$ stores (endoplasmic reticulum, ER). A major breakthrough in the field was the identification of the two essential molecular players in SOCE: STIM1, a $\mathrm{Ca}^{2+}$ sensor in the ER, and Orai1, a $\mathrm{Ca}^{2+}$ permeable channel in the plasma membrane. SOCE is also well-documented in skeletal muscle where it limits muscle fatigue during repetitive stimulation. Also in muscle SOCE is mediated by interactions between STIM1 and Orai1 channels. ${ }^{1}$ However, the precise subcellular location of STIM1Orai1 complexes in skeletal muscle is still debated. We discovered that exercise in mice drives formation of new junctions between stacks of sarcoplasmic reticulum (SR) cisternae and transverse-tubules (TTs) containing respectively STIM1 and Orai1. We proposed that these previously unidentified SR-TT junctions function as $\mathrm{Ca}^{2+}$ Entry Units (CEUs), providing a preferential pathway for rapid reuptake of $\mathrm{Ca}^{2+}$ into the SR during repetitive muscle activity. ${ }^{2}$ We also discovered that CEUs are dynamic, as they increase in number and size during exercise, while reduce following recovery. ${ }^{3}$

For many years the role of extracellular $\mathrm{Ca}^{2+}$ for skeletal muscle function was ignored. Our recent work represents a pioneer study that identified exercise-driven dynamic formation of new intracellular structures, a mechanism potentially quite important for the delay of muscle fatigue. As altered SOCE activity contributes to muscle dysfunction in ageing and various myopathies, our findings may also have implications for the understanding of mechanisms involved in muscular dysfunction.

Keywords: $\mathrm{Ca}^{2+}$ entry unit (CEU); sarcoplasmic reticulum (SR); store-operated $\mathrm{Ca}^{2+}$ entry (SOCE).

\section{References}

1. Lyfenko AD, Dirksen RT. Differential dependence of store-operated and excitation-coupled Ca2 + entry in skeletal muscle on STIM1 and Orai1. J Physiol. 2008 Oct 15;586(20):4815-24. doi: 10.1113/jphysiol. 2008.160481. Ерub 2008 Sep 4.

2. Boncompagni S, Michelucci A, Pietrangelo L, Dirksen RT, Protasi F. Exercise-dependent formation of new junctions that promote STIM1-Orai1 assembly in skeletal muscle. Sci Rep. 2017 Oct 27;7(1):14286. doi: 10.1038/s41598-017-14134-0.
3. Michelucci A, Boncompagni S, Pietrangelo L, García-Castañeda M, Takano T, Malik S, Dirksen RT, Protasi F. Transverse tubule remodeling enhances Orai1-dependent $\mathrm{Ca}^{2+}$ entry in skeletal muscle. Elife. 2019 Oct 28;8:e47576. doi: 10.7554/eLife.47576.

$$
* * * * *
$$

\#36. May 27, 2021

\section{Assessing skeletal muscle structure with stereology}

\section{Hans Hoppeler}

Department of Anatomy, University of Bern, Bern, Switzerland

* Hans Hoppeler: hoppeler@ana.unibe.ch

Stereology in a narrow sense is defined as a body of methods that allow for extraction of quantitative 3dimensional information from 2-dimensional planar sections of tissue or materials. Stereology uses principles of statistics and of stochastic geometry and, if properly executed, provides unbiased quantitative data with regard to volume, length, surface, number and distribution of structures of interest. Modern stereological techniques are "design-based" and work without any assumptions about the shape, size, orientation or distribution of the objects under scrutiny. The estimated quantities of interest are thus free of bias. Skeletal muscle poses a significant challenge to stereological analysis because of the extremely anisotropic organization of the tissue elements. Muscle fibers are essentially unidirectional arranged cylinders with all tissue elements surrounding muscle fibers or laying within muscle fibers similarly oriented to a varying degree with regard to the longitudinal axis of the muscle fibers. In order to obtain an unbiased estimate of any structural quantity of interest it is essential that muscle tissue be sectioned with an IUR (independent uniform random) procedure, which guarantees that sections for analysis are random in all three planes. ${ }^{1}$ This approach is appropriate and efficient for estimating volumes and surfaces such as mitochondrial volumes and membrane surfaces. However, for measuring aspects of capillarity the IUR approach has serious drawbacks. Due to capillary anisotropy parameter estimates may have unfeasibly large coefficients of variation. It may therefore be of advantage to use a 3-dimensional approach such as microangioCT of muscle tissue volumes. ${ }^{2}$ To estimate numbers of "particles" in muscle tissue such as cell nuclei or satellite cells is a particular challenge. It is necessary to adhere to rigorous counting methods using appropriate counting frames, and to do that on two consecutive sections of a known distance to obtain an unbiased estimate of the particle of interest. ${ }^{3}$

Keywords: Skeletal muscle; stereology; IUR; dissector; sampling. 


\section{References}

1. Nyengaard JR, and Gundersen HJG. The Isector: A Simple and Direct Method for Generating Isotropic, Uniform Random Sections from Small Specimens. J. Microsc., 1992, 165, pp 427-431.

2. Ruslan $H$, Haberthür $D$, Djonov $V$. Ex vivo microangioCT: Advances in microvascular imaging. Vascular pharmacology, 2019, 112, pp 2-7. Elsevier 10.1016/j.vph.2018.09.003.

3. Sterio DC. The unbiased estimation of number and sizes of arbitrary particles using the dissector. $J$. Microsc. 1984, May;134(Pt 2):127-36.m doi: 10.1111/j.1365-2818.1984.tb02501.x.

$$
* * * * *
$$

\#37. May 27, 2021

\section{Exertional Heat Stroke: the possible role of external $\mathrm{Ca}^{2+}$}

Barbara Girolami, ${ }^{*}$ Matteo Serano, Antonio Michelucci, Laura Pietrangelo, Feliciano Protasi

CAST, Center for Advanced Studies and Technology; University G D'Annunzio of Chieti-Pescara, Italy

* Barbara Girolami: barbara.girolami@unich.it

Exertional/Environmental Heat Strokes (EHSs) are hyperthermic crises triggered by strenuous physical exercise and/or exposure to environmental heat, which are caused by an altered intracellular $\mathrm{Ca}^{2+}$ homeostasis in muscle. ${ }^{1,2}$ We recently demonstrated that a single bout of exercise on treadmill leads to formation of Calcium Entry Units (CEUs), intracellular junctions that promote interaction between STIM1 and Orai1, the two proteins that mediate Store-Operated $\mathrm{Ca}^{2+}$ Entry (SOCE). SOCE is a mechanism that is activated during muscle fatigue and that allows recovery of extracellular $\mathrm{Ca}^{2+}$ during prolonged activity.,4 The hypothesis underlying this project is that assembly of CEUs during prolonged exercise may predispose to Exertional Heat Stroke (EHS), when exercise is performed in challenging environmental conditions. To test this hypothesis, we used different groups of mice: pre-exercised wild type (WT) mice, in which assembly of CEUs was induced by an incremental running protocol (as in ref. 3) and two genetically modified mice which are known to be hypersensitive to heat (RyR1- G2435R and CASQ1-null mice).The animals were subjected to an exertional stress (ES) protocol consisting of an incremental 45min treadmill run at $34^{\circ} \mathrm{C}$ and $40 \%$ humidity (for CASQ1-null mice the protocol was reduced to $30 \mathrm{~min}$ to avoid lethality). In all 3 groups of mice we tested the internal temperature reached at the end of the ES protocol was higher (RyR1-G2435R: $39.1^{\circ} \mathrm{C} \pm 0.85$; CASQ1-null: $38.4^{\circ} \mathrm{C} \pm 0.07$; WT pre-exercised: $38.8^{\circ} \mathrm{C} \pm 0.48$ ) compared to WT control that were not pre-exercised $\left(37.9^{\circ} \mathrm{C} \pm 0.17\right)$. We then analyzed muscles by electron microscopy (EM) and verified that: a) the number/area of CEUs is higher in pre-exercised WT mice vs. control
(Control: 2,0 \pm 0,6; Exercised 1h: 9,7 $\pm 1,0$ ); b) in RyR1G2435R and CASQ1-null mice CEUs are constitutively present. The data collected indicates that presence of CEUs correlates with a greater increase in body temperature and could in principle predispose to EHS when exercise is performed in challenging environmental conditions.

Keywords: calcium entry unit (CEU), heat-stroke, storeoperated Ca2+ entry (SOCE).

\section{References}

1. Bouchama A, Knochel JP. Heat stroke. $N$ Engl J Med. 2002 Jun 20;346(25):1978-88. doi: 10.1056/NEJM ra011089.

2. Durham WJ, Aracena-Parks P, Long C, Rossi AE, Goonasekera SA, Boncompagni S, Galvan DL, Gilman CP, Shirokova N, Protasi F, Dirksen RT, Hamilton SL. RYR1 S-Nitrosilation underlies environmental heat stroke and sudden death in Y522S RyR1 knockin mice. Cell. 2008 Apr 4;133(1):53-65. doi: 10.1016/j.cell.2008.02.042.

3. Boncompagni S, Michelucci A, Pietrangelo L, Dirksen $R T, F$. Protasi. Exercise-dependent formation of new junctions that promote STIM1-Orai1 assembly in skeletal muscle. Sci Rep. 2017 Oct 27;7(1):14286. doi: 10.1038/s41598-017-14134-0.

4. Protasi F, Pietrangelo L, Boncompagni S. Calcium entry units (CEUs): perspectives in skeletal muscle function and disease. J Muscle Res Cell Motil. 2020 Aug 18. doi: 10.1007/s10974-020-09586-3. Online ahead of print.

$* * * * *$

\#38. May 27, 2021

Morphological study of TNPO3 and SRSF1 interaction during myogenesis by combining confocal, structured illumination and electron microscopy analyses

Roberta Costa (1,2), Maria Teresa Rodia (1,2), Nicoletta Zini (3,4), Valentina Pegoraro (5), Roberta Marozzo (5), Cristina Capanni $(3,4)$, Corrado Angelini (5), Giovanna Lattanzi $(3,4)$, Spartaco Santi $(3,4)$, Giovanna Cenacchi $(1,2)^{*}$

(1) Department of Biomedical and Neuromotor Sciences - DIBINEM, University of Bologna, Bologna, Italy; (2) Center of Applied Biomedical Research CRBA, University of Bologna, St. Orsola Hospital, Bologna, Italy; (3) CNR - National Research Council of Italy, Institute of Molecular Genetics "Luigi Luca Cavalli-Sforza", Unit of Bologna, Bologna, Italy; (4) IRCCS Istituto Ortopedico Rizzoli, Bologna, Italy; (5) Neuromuscular Unit, Neurobiology Research group, IRCCS San Camillo Hospital, Venice, Italy.

* Giovanna Cenacchi: giovanna.cenacchi@unibo.it 
Limb girdle muscular dystrophy D2 (previously LGMD1F) is a rare neuromuscular disease due to a heterozygous mutation in the TNPO3 gene coding for Transportin 3 (TNPO3). TNPO3 is an importin that shuttles, from the cytoplasm to the nucleus, the serine/arginine-rich proteins (SR-proteins) among which the splicing factor SRSF1. The SR family includes proteins involved in mRNA metabolism and essential splicing factors that, regulating alternative splicing, contribute to post transcriptional gene regulation. In this way they affect the proteomic diversity in muscle, contributing to the control of satellite cell fate during muscle differentiation and helping the maintenance of healthy muscle. Since the strict interaction between SRSF1 and TNPO3, a mutation in TNPO3 could downstream dysregulate SRSF1 function, affecting splicing and causing abnormalities in fibers that contribute to LGMD D2 pathogenesis. We analyzed if myogenic differentiation could be modulated by interactions between SRSF1 and TNPO3. The different steps of myogenesis have been monitored in C2C12 cells and TNPO3 expression have been investigated by confocal and electron microscopy, showing that it increases in nuclei of differentiated myotubes. The interactions of TNPO3 and SRSF1 have been quantitatively evaluated by a structured illumination microscope: SRSF1 remains localized in the nucleus, while TNPO3 decreases in the cytoplasm appearing strongly clustered in the nucleus of myotubes, suggesting that it could act in the proteomic network necessary during myogenesis. These data stress the role of TNPO3 as a carrier of SRSF1 in crucial steps of myogenic differentiation and could help to clarify the pathogenic mechanisms of LGMD D2.

Keywords: TNPO3; SRSF1; Myogenesis.

\section{References}

1. Maertens GN, Cook NJ, Wang W, Hare S, Gupta SS, Öztop I, Lee K, Pye VE, Cosnefroy O, Snijders AP, KewalRamani VN, Fassati A, Engelman A, Cherepanov $P$.. Structural basis for nuclear import of splicing factors by human Transportin 3. Proc Natl Acad Sci USA. 2014;111:2728-2733. doi: 10.1073/pnas.13207 55111.

2. Long JC, Caceres JF. The SR protein family of splicing factors:master regulators of gene expression. Biochem J. 2009;417:15-27. Doi: 10.1042/bj200 81501.

3. Torella A, Fanin M, Mutarelli M, Peterle E, Del Vecchio Blanco F, Rispoli R, Savarese M, Garofalo A, Piluso G, Morandi L, Ricci G, Siciliano G, Angelini C, Nigro V. Next-generation sequencing identifies transportin 3 as the causative gene for LGMD1F. PLoS One. 2013;8:e63536. doi:10.1371 /journ al.pone.0063536.

4. Costa R, Rodia MT, Vianello S, Santi S, Lattanzi G, Angelini C, Pegoraro E, Cenacchi G. Transportin 3 (TNPO3) and related proteins in limb girdle muscle dystrophy D2 muscle biopsies: a morphological confocal microscopy study and pathogenetic hypothesis. Neuromuscul Disord. 2020. 2020 Aug;30(8):685-692. doi: 10.1016/j.nmd.2020.05.006. Epub 2020 May 26.
\#39. May 27, 2021

Exploiting live imaging to track nuclei during myoblast differentiation and fusion in wild type and dystrophic mouse models

Mauro Passaretti (1,2), Giorgia Careccia (1,2), Federica Colombo (2,3), Mario Tirone (2), Alessandra Agresti (2), Marco Bianchi (1,2), Samuel Zambrano (1,2), Emilie Venereau (2)

(1) San Raffaele University, Milan, Italy; (2) Division of Genetics and Cell Biology, IRCCS San Raffaele Scientific Institute, Milan, Italy; (3) Department of Electronics, Information and Bioengineering, Politecnico di Milano, Italy.

* Mauro Passaretti: passaretti.mauro@hsr.it

Skeletal muscle differentiation is a highly dynamic process and correct nuclear positioning within myofibers is essential for proper muscle regeneration and function. ${ }^{1}$ Hence, the mobility of myoblasts and of their nuclei appears to be a critical parameter to evaluate myofiber formation, but this highly dynamical process cannot be analyzed by the standard procedure that relies on fixed cells analyzed by immunofluorescence. We developed a method to record myoblast differentiation and myotube formation by time-lapse microscopy and we used a software to perform quantitative analyses from the automatic tracking of myoblast nuclei. ${ }^{2}$ Our current objective is to exploit this method to compare nuclear and cell dynamics of myoblasts, isolated from wild type and dystrophic mice, during differentiation. To extract information on nuclei/cell movements data from the live cell imaging experiments, we are performing measurement of multiple parameters for each tracked nucleus (e.g. velocity, trajectory length). Our aim is to give new insights into the dynamics of myofibers formation and into the pathogenesis of muscular dystrophies.

Keywords: nuclear positioning; myofibers; live imaging; cell tracking; muscular dystrophy.

\section{References}

1. Roman W, Gomes ER. Nuclear positioning in skeletal muscle. Semin Cell Dev Biol. 2018 Oct;82:51-56. doi: 10.1016/j.semcdb.2017.11.005. Epub 2017 Dec 11.

2. Careccia, Colombo F, Tirone M, Agresti A, Bianchi $M E$, Zambrano S, Vénéreau E. Exploiting Live Imaging to Track Nuclei During Myoblast Differentiation and Fusion. J Vis Exp. 2019 Apr 13;(146). doi: 10.3791/58888 
\#40. May 28, 2021

\section{Regeneration of aged human muscle}

\section{Terence A. Partridge}

UCL Great Ormond Street Institute of Child Health, London, UK

* Terence Partridge: t.partridge@ucl.ac.uk

It is widely speculated that age related muscle atrophy is a result of failure to maintain of muscle in the face of loss by damage during day-to-day interaction with the environment. This failure is, in turn, associated with a loss of regenerative capacity by the satellite cell. To examine this idea, we used a previously established model in which fragments of human muscle regenerate when grafted into immunodeficient mouse hosts. ${ }^{1}$ Grafts were obtained during autopsy of cadavers of individuals of a range of ages at death. We found strong regeneration of muscle fragments across the entire range of ages up to 91, suggesting that there was little evidence of a decline of regenerative vigor with age.

Keywords: regeneration; aging; human; muscle; immunodeficient mouse hosts.

\section{References}

1. Zhang Y, King OD, Rahimov F, Jones TI, Ward CW, Kerr JP, Liu N, Emerson CP Jr, Kunkel LM, Partridge TA, Wagner KR. Human skeletal muscle xenograft as a new preclinical model for muscle disorders. Hum Mol Genet. 2014 Jun 15;23(12):3180-8. doi: 10.1093/hmg/ddu028. Epub 2014 Jan 22.

$$
* * * * *
$$

\#41. May 28, 2021

Nuclear misplacement and muscle regeneration in long-term denervated human muscles before and after FES

\section{Ugo Carraro $(1,2,3)$}

(1) Department of Biomedical Sciences of the University of Padova, Italy; (2) CIR-Myo - Myology Centre, University of Padova, Italy; (3) A\&C MioniCarraro Foundation for Translational Myology, Padova, Italy

\section{* Ugo Carraro: ugo.carraro@unipd.it}

After a complete spinal cord injury that damage soma and axons of the lower motor neuron (LMN), the permanent denervated human muscle fibers lose completely the myofibrillar apparatus and the coil distribution of myonuclei that are relocated in groups (nuclear clumps) in the center of severely atrophic muscle fibers. ${ }^{1} \mathrm{Up}$ to two years of LMN denervation the muscle fibers with nuclear clumps are very seldom, but then severely atrophic muscle fibers are frequent in muscle biopsies harvested three to six years after permanent denervation. Indeed, the percentage increased to $27 \pm 9 \%(\mathrm{p}<0.001)$, and then abruptly decreased from the 6th year onward, when fibrosis takes over to neurogenic muscle atrophy. Immunohistochemical analyses shown that nuclear misplacements occurred in both fast and slow muscle fibers, while seldom tough continuous events of myofibers regeneration occurred presenting central nuclei and embryonic myosin heavy chain positive staining. Those events were present at lower level in muscle biopsies harvested after two years of Functional Electrical Stimulation., ${ }^{5,6}$ In conclusion, human muscle fibers survive permanent denervation much longer than

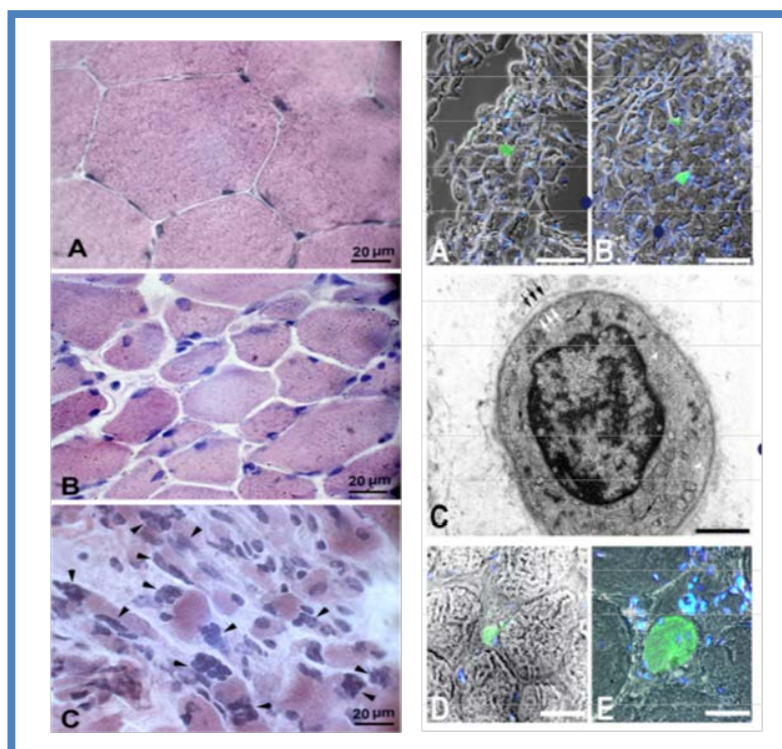

Fig 1. Left Panel: Nuclear clumps are predominant after many years of denervation in human muscle fibers. ${ }^{1}$ A, Normal muscle; B, 0.8- year denervated muscle characterized by atrophy of myofibers and seldom central nuclei; C, 4.1year denervated muscle showing severely atrophic myofibers in which contractile apparatus is almost absent and three or more clumped nuclei can be identified in oblique muscle sections. Scale bars: $A, B, C=20 \mu \mathrm{m}$. Right panel: Regenerative events in long-term denervated human muscle without (A and $B$ ) and after ( $D$ and $E$ ) several years of homebased Functional Electrical Stimulation (hbFES). Note the extent of recovery of myofiber after years of hbFES. ${ }^{2-6} A$ and $B, 0.7$ and 8.7 years of denervation; C, Electron micrograph of a regenerating myotube in longterm denervated muscle, that is surrounded by two layers of basal lamina; D, 2-year denervation followed by 4.3 years of hbFES training; E, 23.3-year denervation followed by 2.7-year hbFES training. Recently regenerated myofibers are stained in green with an antibody anti-embryonic myosin heavy chain. Bars: $A, B=100 \mu \mathrm{m} ; \quad C=2.0 \mu \mathrm{m}: D, E=$ $50 \mu \mathrm{m}$. 
generally accepted and relocation of myonuclei is a general behavior in long term denervated muscle fibers.

Keywords: Nuclear misplacement; muscle regeneration; long-term denervated human muscles; Functional Electrical Stimulation.

\section{References}

1. Carraro U, Kern H. Severely Atrophic Human Muscle Fibers With Nuclear Misplacement Survive Many Years of Permanent Denervation. Eur J Transl Myol. 2016 Jun 13;26(2):5894. doi: 10.4081/ejtm.2016.5894. eCollection 2016 Jun 13. PMID: 27478559 Free PMC article.

2. Kern H, Boncompagni S, Rossini K, Mayr W, Fanò G, Zanin ME, Podhorska-Okolow M, Protasi F, Carraro U Long-term denervation in humans causes degeneration of both contractile and excitationcontraction coupling apparatus, which is reversible by functional electrical stimulation (FES): a role for myofiber regeneration? J Neuropathol Exp Neurol. 2004 Sep;63(9):919-31. doi: 10.1093/jnen/63.9.919.

3. Carraro U, Rossini K, Mayr W, Kern H. Muscle fiber regeneration in human permanent lower motoneuron denervation: relevance to safety and effectiveness of FES-training, which induces muscle recovery in SCI subjects. Artif Organs. 2005 Mar;29(3):187-91. doi: 10.1111/j.1525-1594.2005.29032.x.

4. Kern H, Salmons S, Mayr W, Rossini K, Carraro U. Recovery of long-term denervated human muscles induced by electrical stimulation.Muscle Nerve. 2005 Jan;31(1):98-101. doi: 10.1002/mus.20149.

5. Kern H, Carraro U, Adami N, Biral D, Hofer C, Forstner C, Mödlin $M$, Vogelauer $M$, Pond A, Boncompagni S, Paolini C, Mayr W, Protasi F, Zampieri S. Home-based functional electrical stimulation rescues permanently denervated muscles in paraplegic patients with complete lower motor neuron lesion. Neurorehabil Neural Repair. 2010 Oct;24(8):709-21. doi: 10.1177/1545968310366129. Epub 2010 May 11.

6. Kern H, Carraro U. Home-Based Functional Electrical Stimulation of Human Permanent Denervated Muscles: A Narrative Review on Diagnostics, Managements, Results and Byproducts Revisited 2020. Diagnostics (Basel). 2020, 10, 529. doi: 10.3390/diagnostics 10080529.

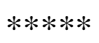

\#42. May 28, 2021

Sertoli cell-secreted factors have promyogenic and antifibrotic properties on human DMD myoblasts with different mutations

Laura Salvadori $(1,2)^{\#}$, Sara Chiappalupi $(2,3)^{\#}$, Iva Arato (3), Francesca Mancuso (3), Mario Calvitti (3), Cristina Marchetti (3), Francesca Riuzzi (2,3), Riccardo Calafiore $(3,4)$, Giovanni Luca $(3,4)$ Guglielmo Sorci $(2,3,4) *$
\# These two authors contribute equally

(1) Department of Translational Medicine, University of Piemonte Orientale, Novara, Italy; (2) Interuniversity Institute of Myology (IIM), Perugia, Italy; (3) Department of Medicine and Surgery, University of Perugia, Perugia, Italy; (4) Centro Biotecnologico Internazionale di Ricerca Traslazionale a indirizzo Endocrino, Metabolico ed Embrio-Riproduttivo (CIRTEMER), Perugia, Italy.

* Guglielmo Sorci: guglielmo.sorci@unipg.it

Duchenne muscular dystrophy (DMD) is a recessive Xlinked lethal disease affecting one over 5,000 live male births in which mutations in the dystrophin gene (DMD) lead to lack of a functional protein resulting in susceptibility of myofibers to rupture during contraction. Muscles of DMD patients or experimental models of DMD show progressive necrosis of the myofibers, chronic inflammation and reactive regeneration, which lead to exhaustion of muscle precursor cell pool and replacement of myofibers with fibrous and fatty tissues. ${ }^{1}$ Although multiple therapeutic approaches have been explored in the last decades and are still under investigation, the standard therapy to DMD remains the use of glucocorticoids despite their limited efficacy and undesired side effects. ${ }^{2}$ We set up a preclinical approach based on the peculiar properties of Sertoli cells (SeC) $)^{3,4}$ a cell type of the seminiferous tubules of the testis in which they favor the maturation of developing germ cells and protect them against the host immune system. Besides creating a physical barrier (the blood-testis barrier), SeC secrete a plethora of trophic and immunomodulatory factors that confer to these cells the ability to survive long time after engraftment, and protect allo- and xenogenic engraftments of tissues and organs. ${ }^{5} \mathrm{SeC}$ isolated from specific pathogens free (SPF) pre-pubertal pigs were encapsulated in highly biocompatible alginate-based microcapsules (MC-SeC) and injected into the peritoneal cavity of mdx mice, an experimental model of DMD, in the absence of pharmacological immunosuppression. ${ }^{3,4} \mathrm{~A}$ single i.p. injection of MC-SeC resulted in amelioration of muscle morphology and performance as a consequence of the secretion by SeC of antiinflammatory factors and heregulin $\beta 1$, an inducer of the dystrophin paralogue, utrophin, opening new routes in the treatment of DMD. However, information is lacking about possible direct effects of $\mathrm{SeC}$ on myoblasts/myotubes. Here, we show that SeC secrete factors able to stimulate cell proliferation in the early phase of the myogenic differentiation process in murine C2C12 and human (healthy and DMD) myoblasts. In DMD myoblasts, SeC-derived factors delayed the expression of the muscle-specific terminal differentiation markers, myogenin and myosin heavy chain (MyHC)-II in the early phase (24h) of the differentiation process; nevertheless, SeC stimulated terminal differentiation (6 days) in these cells, suggesting that the promitogenic activity of SeC does not affect the myogenic potential. 
Moreover, SeC inhibit the expression of the myofibroblast transdifferentiation markers, COL1A1, FN1 and CTGF/CCN2 in DMD myoblasts, suggesting an antifibrotic effect of the SeC-derived factors. Finally, SeC induced utrophin expression in preformed DMD myotubes regardless of the mutation, with the same mechanism reported in dystrophic mice. Altogether, these results further support the use of MC-SeC or SeCderived factors in the treatment of DMD patients, and suggest that SeC-based approaches might be useful also in improving the early phase of muscle regeneration, during which myoblasts have to proliferate in order to reach the critical amount required to replace the damaged muscle mass.

Keywords: Duchenne muscular dystrophy; Sertoli cell; muscle differentiation; fibrosis.

\section{References}

1. Davies KE, Nowak KJ. Molecular mechanisms of muscular dystrophies: old and new players. Nat Rev Mol Cell Biol. 2006;10:762-73. doi: 10.1038/nrm 2024

2. Muntoni F, Fisher I, Morgan, JE, Abraham D. Steroids in Duchenne muscular dystrophy: from clinical trials to genomic research. Neuromuscul. Disord. 2002;1:S162-5. doi: 10.1016/s0960-8966 (02)00101-3

3. Chiappalupi S, Luca G, Mancuso F, Madaro L, Fallarino F, Nicoletti C, Calvitti $M$, Arato I, Falabella F, Salvadori L, Di Meo A, Bufalari A, Giovagnoli S, Calafiore $R$, Donato $R$, Sorci $G$. Intraperitoneal injection of microencapsulated Sertoli cells restores muscle morphology and performance in dystrophic mice. Biomaterials. 2016;75:313-26. doi:10.1016/j.biomaterials. 2015. 10.029

4. Chiappalupi S, Luca G, Mancuso F, Madaro L, Fallarino F, Nicoletti C, Calvitti $M$, Arato I, Falabella F, Salvadori L, Di Meo A, Bufalari A, Giovagnoli S, Calafiore R, Donato R, Sorci G. Effects of intraperitoneal injection of microencapsulated Sertoli cells on chronic and presymptomatic dystrophic mice. Data in Brief. 2015;5:1015-21. doi:10.1016/j.dib.2015.11.016

5. Chiappalupi S, Salvadori L, Luca G, Riuzzi F, Calafiore R, Donato R, Sorci G. Do porcine Sertoli cells represent an opportunity for Duchenne muscular dystrophy? Cell Proliferation. 2019;26:e12599; doi: 10.1111/cpr.12599.
\#43. May 28, 2021

Invited Lecture: The skeletal muscle satellite cell: still young and fascinating at 60

\section{Zipora Yablonka-Reuveni}

Department of Biological Structure, University of Washington School of Medicine, Seattle, WA, USA

* Zipora Yablonka-Reuveni: reuveni@uw.edu

The skeletal muscle satellite cell was first described in 1961 based on its anatomical location between the myofiber plasma and basement membranes. Soon after the two 1961 independent reports by Alexander Mauro and Bernard Katz, the satellite cells acquired candidacy as the source of myogenic cells needed for myofiber growth and repair throughout life. Cultures of isolated myofibers, and subsequently, transplantation of single myofibers, indeed have demonstrated that satellite cells were myogenic progenitors. Identification of distinctively expressed molecular markers, in particular Pax7, has facilitated detection of satellite cells using light microscopy. With time, the satellite cells have also gained the status of "myogenic stem cells" being capable

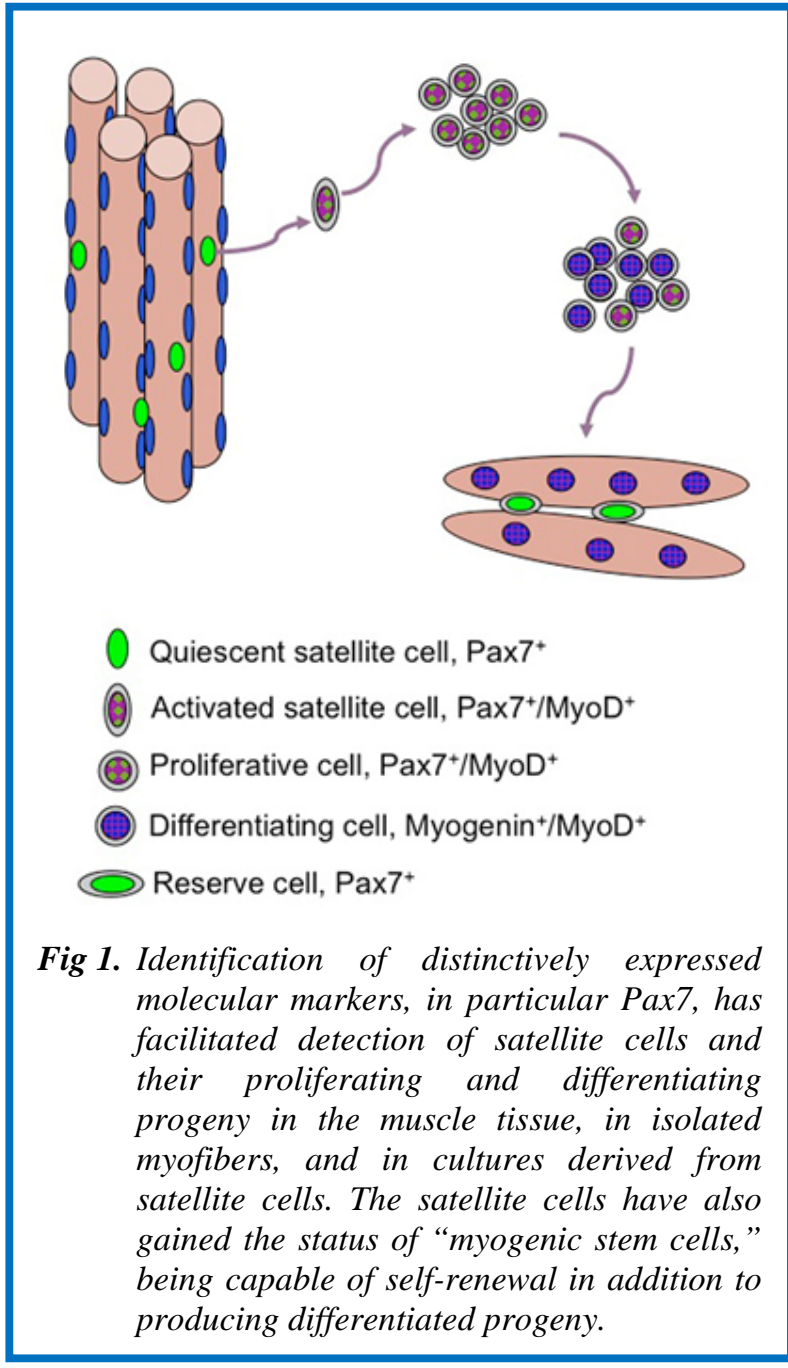


of self-renewal in addition to producing differentiated progeny. Despite the remarkable progress made since the discovery of satellite cells, researchers have looked for alternative cells with myogenic capacity that could potentially be utilized for whole body cell-based therapy of skeletal muscle. Yet, advances made with genetic mouse models that permit inducible ablation of satellite cells, have certainly reaffirmed the satellite cell's indispensable role in muscle repair. Genetic lineage tracing has also helped in establishing that the satellite cells strictly provide myogenic cells, but other cell lineages may act in concert with satellite cells in supporting muscle health. Despite much research, the extent of contribution of satellite cells to muscle maintenance in adult and old ages and during muscle hypertrophy and atrophy, has still not been fully resolved. Likewise, the molecular mechanisms of satellite cell recruitment and the proliferation-differentiation-renewal interplay awaits further studies. Another, yet to be resolved aspect, encompasses the satellite cell embryological origin in different muscles and the molecular regulation of the cell's progression to the satellite cell phenotype. Thus, on the $60^{\text {th }}$ anniversary since its discovery, the satellite cell continues inspiring us to unmask its fascinating secrets. In my talk, I will present some of the historical milestones in the satellite cell field and discuss some published and not-published aspects of my years in myogenesis.

Keywords: satellite cell; pericyte; fibro/adipocyte; diaphragm; extraocular muscle; Pax3/7.

\section{References}

1. Grounds MD, Yablonka-Reuveni Z. Molecular and cell biology of skeletal muscle regeneration. Mol Cell Biol Hum Dis Ser. 1993;3:210-56. doi: 10.1007/97894-011-1528-5-9.

2. Yablonka-Reuveni Z. Development and postnatal regulation of adult myoblasts. Microsc Res Tech. 1995; 30: 366-380. 10.1002/jemt.1070300504.

3. Zammit PS, Partridge TA, Yablonka-Reuveni Z. The skeletal muscle satellite cell: the stem cell that came in from the cold. J Histochem Cytochem. 2006 Nov; 54: 1177-1191. doi: 10.1369/jhc.6R6995.2006.

4. Scharner J, Zammit PS. The muscle satellite cell at 50: the formative years. Skelet Muscle. 2011; 1: 28. doi: 10.1186/2044-5040-1-28

5. Yablonka-Reuveni, Z. The skeletal muscle satellite cell: still young and fascinating at 50. J Histochem Cytochem. 2011; 59, 1041-1059. doi: 10.1369/ 0022155411426780.

6. Relaix F, Zammit PS. Satellite cells are essential for skeletal muscle regeneration: the cell on the edge returns centre stage. Development 2012; 139: 2845 2856. doi: 10.1242/dev.069088.

7. Baghdadi MB, Tajbakhsh S. Regulation and phylogeny of skeletal muscle regeneration. Dev Biol. 2018; 433: 200-209. doi: 10.1016/j.ydbio.2017. 07.026.
8. Morgan J, Partridge T. Skeletal Muscle in Health and Disease. Dis Model Mech. 2020; 13: dmm042192. doi: 10.1242/dmm.042192.

$$
* * * * *
$$

\#44. May 28, 2021

Are the mechanisms of muscle growth and regeneration mutually compatible?

\section{Terence A. Partridge}

\section{UCL Great Ormond Street Institute of Child Health, London, UK}

* Terence Partridge: t.partridge@ucl.ac.uk

Growth and regeneration of muscle both entail successive proliferation, differentiation and fusion of satellite cells, overseen by an orderly sequence of expression of Myogenic Regulatory Factors. However, detailed investigations of myogenesis muscle growth and regeneration reveal fundamentally different dynamics between the two, ${ }^{1}$ bringing into question the issue of whether they can efficiently operate contemporaneously within the same tissue. Measurement of myonuclear increase in fibers of the EDL muscle during the burst of postnatal growth in the mouse corresponds closely to the model of serial asymmetric divisions of associated satellite cells, ${ }^{2,3}$ with one daughter cell from each division entering myogenesis, the other remaining proliferative. This process is identical in the mdx mouse and its wildtype equivalent. In contrast, pulse-chase experiments reveal that each episode of muscle degeneration, provokes a rapid 2-3 day burst of proliferation of satellite cells to repair the damaged muscle, of a size that can only be achieved by a series of predominantly symmetric divisions of myoblasts committed to myogenic differentiation. Two such disparate processes of myogenic dynamics must involve radically different overall regulatory processes of control of satellite cell proliferation that if operating within the same tissue are likely to interfere with one another. In the mdx mouse, the main phase of myogenic growth largely precedes the onset of myopathic disease whereas in DMD boys, the two processes co-exist for 18 years, perhaps accounting for the milder clinical course in the mdx mouse by comparison with the progressively severe disease seen in DMD boys.

Keywords: symmetric/asymmetric cell division; stem cell proliferation; muscle growth; muscle regeneration.

\section{References}

1. Duddy W, Duguez S, Johnston H, Cohen TV, Phadke A, Gordish-Dressman H, Nagaraju K, Gnocchi V, Low S, Partridge T. Muscular dystrophy in the $\mathrm{mdx}$ mouse is a severe myopathy compounded by hypotrophy, hypertrophy and hyperplasia.. Skelet Muscle. 2015 May 1;5:16. doi: 10.1186/s13395-015-0041-y. eCollection 2015. 
2. Novak JS, Hogarth MW, Boehler JF, Nearing M, Vila $M C$, Heredia $R$, Fiorillo AA, Zhang A, Hathout $Y$, Hoffman EP, Jaiswal JK, Nagaraju K, Cirak S, Partridge TA. Myoblasts and macrophages are required for therapeutic morpholino antisense oligonucleotide delivery to dystrophic muscle. Nat Commun. 2017 Oct 16;8(1):941. doi: 10.1038/s41467-017-00924-7.

3. Mazala DA, Novak JS, Hogarth MW, Nearing M, Adusumalli P, Tully CB, Habib NF, Gordish-Dressman $H$, Chen YW, Jaiswal JK, Partridge TA. TGF-beta-driven muscle degeneration and failed regeneration underlie disease onset in a DMD mouse model. JCI Insight. 2020 Mar 26;5(6):e135703. doi: 10.1172/jci.insight.135703.).

\section{$* * * * *$}

\#45. May 28, 2021

Novel roles for satellite cells in muscle adaptation and aging Novel roles for satellite cells in muscle adaptation and aging

\section{Charlotte A. Peterson}

Center for Muscle Biology, University of Kentucky, Lexington, KYUSA

\section{* Charlotte A. Peterson: cpete4@uky.edu}

Satellite cells are activated to proliferate and regenerate damaged myofibers and contribute nuclei to growing myofibers in response to hypertrophic stimuli. We showed that activated satellite cells/myogenic progenitor cells (MPCs) may also regulate remodeling of the extracellular matrix during hypertrophic growth through interaction with fibroblasts. We showed that in vitro, MPC-derived extracellular vesicles (EVs) down-regulate fibroblast collagen expression and depletion of MPC EVderived microRNAs, particularly miR-206, eliminates this effect. Muscle atrophy occurs with age (sarcopenia), and both muscle regeneration and the hypertrophic response are blunted in old age, associated with a decline in satellite cell abundance. However, whereas depleting satellite cells in mouse muscle throughout adulthood does not exacerbate sarcopenia, it does reduce muscle adaptability to lifelong exercise. Senescent cells accumulate in young adult and aged muscle in response to both injury and a hypertrophic stimulus (mechanical overload), which persist in aged muscle. Senolytics preferentially improve muscle regeneration and hypertrophy, and increase satellite cell abundance in old muscle, whereas senolytics may have detrimental effects in young muscle. Additional molecular and cellular adaptations to senolytics in old muscle will be presented. Acknowledgements: This work was supported by grants from NIA and NIAMS.

Keywords: satellite cells; hypertrophy; aging; regeneration; senescence.

References
1. Fry CS, Lee JD, Mula J, Kirby TJ, Jackson JR, Liu F, Yang L, Mendias CL, Dupont-Versteegden EE, McCarthy JJ, Peterson CA. Inducible depletion of satellite cells in adult, sedentary mice impairs muscle regenerative capacity without affecting sarcopenia. Nat Med. 2015 Jan;21(1):76-80. doi: 10.1038/nm. 3710. Epub 2014 Dec 15.

2. Fry CS, Kirby TJ, Kosmac K, McCarthy JJ, Peterson CA. Myogenic Progenitor Cells Control Extracellular Matrix Production by Fibroblasts during Skeletal Muscle Hypertrophy. Cell Stem Cell. 2017 Jan 5;20(1):56-69. doi: 10.1016/j.stem.2016. 09.010. Epub 2016 Nov 10.

3. Englund DA, Murach KA, Dungan CM, Figueiredo VC, Vechetti IJ Jr, Dupont-Versteegden EE, McCarthy JJ, Peterson CA. Depletion of resident muscle stem cells negatively impacts running volume, physical function, and muscle fiber hypertrophy in response to lifelong physical activity. Am J Physiol Cell Physiol. 2020 Jun 1;318(6):C1178-C1188. doi: 10.1152/ajpcell.00090.2020. Epub 2020 Apr 22.

4. Murach KA, Vechetti IJ Jr, Van Pelt DW, Crow SE, Dungan CM, Figueiredo VC, Kosmac K, Fu X, Richards CI, Fry CS, McCarthy JJ, Peterson CA. Fusion-independent satellite cell communication to muscle fibers during load-induced hypertrophy. Function (Oxf). 2020;1(1):zqaa009. doi: 10.1093/ function/zqaa009. Epub 2020 Jul 6.

$$
* * * * *
$$

\#46. May 28, 2021

Tetraspanin CD82 is necessary for muscle stem cell activation and supports dystrophic muscle function

Arielle Hall (1), Tatiana Fontelonga (1), Alec Wright (1), Kathlynn Bugda Gwilt (2), Jeffrey Widrick (1), Alessandra Pasut (3), Francesco Villa (4), Cynthia K. Miranti (5), Devin Gibbs (6), Evan Jiang (7), Hui Meng (8), Michael Lawlor (8), Emanuela Gussoni $(1,9)$ *

(1) Division of Genetics and Genomics, Boston Children's Hospital, USA; (2) Division of Gastroenterology, Hepatology and Nutrition, Boston Children's Hospital, USA; (3) Laboratory of Angiogenesis and Vascular metabolism, Center for Cancer Biology, Leuven, Belgium; (4) F.M. Kirby Neurobiology Center, Boston Children's Hospital, USA; (5) Department of Cellular and Molecular Medicine, University of Arizona College of Medicine, USA; (6) Molecular Biology Institute, UCLA, USA; (7) The University of Pennsylvania, College of Arts and Sciences, USA; (8) Department of Pathology and Laboratory Medicine and Neuroscience Research Center, Medical College of Wisconsin, USA; (9) The Stem Cell Program at Boston Children's Hospital, USA. *Emanuela Gussoni: emanuela.gussoni@enders.tch.harvard.edu 
Tetraspanins are a family of proteins known to assemble protein complexes at the cell membrane. They are thought to play diverse cellular functions in tissues by modifying protein-binding partners, thus bringing complexity and diversity in their regulatory networks. Previously, we identified the tetraspanin KAI/CD82 as a prospective marker for human muscle stem cells. CD82 expression appeared decreased in human Duchenne muscular dystrophy (DMD) muscle, suggesting a functional link to muscular dystrophy, yet whether this decrease is a consequence of dystrophic pathology or a compensatory mechanism in an attempt to rescue muscle from degeneration is currently unknown. We studied the consequences of loss of CD82 expression in normal and dystrophic skeletal muscle and examined the dysregulation of downstream functions in mice aged up to 1 year. Expression of CD82 is important to sustain satellite cell activation, as in its absence there is decreased cell proliferation and less efficient repair of injured muscle. Loss of CD82 in dystrophic muscle leads to a worsened phenotype compared to control dystrophic mice, with decreased pulmonary function, myofiber size, and muscle strength. Mechanistically, decreased myofiber size in $\mathrm{CD}^{-/-}$dystrophic mice is not due to altered PTEN/AKT signaling, although increased phosphorylation of mTOR at Ser2448 was observed. Basal CD82 expression is important to dystrophic muscle, as its loss leads to significantly weakened myofibers and impaired muscle function, accompanied by decreased satellite cell activity that is unable to protect and repair myofiber damage.

Keywords: Tetraspanin; CD82; muscular dystrophy.

\section{References}

1. Rubinstein E. The complexity of tetraspanins. Biochem Soc Trans. 2011 Apr;39(2):501-5. doi: 10.1042/ BST0390501.

2. Alexander MS, Rozkalne A, Colletta A, Spinazzola JM, Johnson S, Rahimov F, Meng H, Lawlor MW, Estrella E, Kunkel LM, Gussoni E. CD82 Is a Marker for Prospective Isolation of Human Muscle Satellite Cells and Is Linked to Muscular Dystrophies. Cell Stem Cell. 2016 Dec 1;19(6):800-807. doi: 10.1016/j.stem.2016. 08.006. Epub 2016 Sep 15.

3. Baghdadi MB, Tajbakhsh S. Regulation and phylogeny of skeletal muscle regeneration. Dev Biol. 2018 Jan 15;433(2):200-209. doi: 10.1016/j.ydbio.2017.07.026. Epub 2017 Aug 12.

4. Im WB, Phelps SF, Copen EH, Adams EG, Slightom $J L$, Chamberlain JS. Differential expression of dystrophin isoforms in strains of mdx mice with different mutations. Hum Mol Genet. 1996 Aug;5(8):1149-53. doi: 10.1093/hmg/5.8.1149.

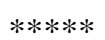

\#47. May 28, 2021

\section{Molecular regulation of muscle stem cell pool size}

William Zofkie (1), Sheryl M Southard (2), Ju-Ryoung Kim (3), Richard Tsika (3), Thomas Braun (4), Christoph Lepper (1)

(1) Department of Physiology and Cell Biology, The Ohio State University, Columbus, OH, USA; (2) SERAXIS Inc, Germantown, MD, USA; (3) Department of Biomedical Sciences, University of Missouri, Columbia, MO, USA; (4) Department of Cardiac Development and Remodeling, Max-Planck-Institute for Heart and Lung Research, Bad Nauheim, Germany

* Christoph Lepper: christoph.lepper@osumc.edu

Skeletal muscle stem cells, i.e. satellite cells, are the essential source of new myonuclei for regeneration of skeletal muscle tissue following acute injury or in chronic degenerative myopathies. Both satellite cell number and regenerative capacity diminish during ageing. Yet, the molecular regulators that govern the sizing of the initial satellite cell pool are currently unknown, and little data exist for satellite cell population homeostasis in the adult. Here, we show that transgenic TEAD1-expressing skeletal muscle features a dramatic satellite cell hyperplasia but surprisingly without affecting muscle tissue size. Such super-numeral satellite cells attain a 'normal' quiescent state, accelerate regeneration, and maintain regenerative capacity over several injuryinduced regeneration bouts. In dystrophic muscle, the TEAD1 transgene also ameliorated the pathology. We further demonstrate that hyperplastic satellite cells accumulate non-cell-autonomously via signal(s) from the TEAD1-expressing myofibers, suggesting that myofiberspecific TEAD1 overexpression activates a physiological signaling pathway(s) that determine initial and homeostatic satellite cell pool size. Applying differential gene expression analysis to the TEAD1 transgenic mouse model, we have identified several candidate molecules for scaling of the satellite cell pool. We are initially focusing our studies on a paracrine signaling ligand, i.e. FGF6, for its muscle-specific expression pattern during development and current lack of data on potential contributions from FGF6 to satellite cell pool scaling during early postnatal development. We are currently investigating SC pool formation in FGF6 germline mutant mice and will discuss our findings. We propose that TEAD1 and its downstream effectors are medically relevant targets for enhancing muscle regeneration.

Keywords: molecular regulation, muscle; stem cell; pool size.

\section{References}

1. Lazure F, Blackburn DM, Corchado AH, Sahinyan K, Karam $N$, Sharanek A, Nguyen D, Lepper C, Najafabadi HS, Perkins TJ, Jahani-Asl A, Soleimani VD. Myf6/MRF4 is a myogenic niche regulator 
required for the maintenance of the muscle stem cell pool. EMBO Rep. 2020 Dec 3;21(12):e49499. doi: 10.15252/embr.201949499. Epub 2020 Oct 13.

2. Li L, Rozo M, Yue S, Zheng X, J Tan F, Lepper C, Fan CM. Muscle stem cell renewal suppressed by Gas1 can be reversed by GDNF in mice. Nat Metab. 2019 Oct;1(10):985-995. doi: 10.1038/s42255-019-01103. Epub 2019 Sep 30.

3. Southard S, Kim JR, Low S, Tsika RW, Lepper C. Myofiber-specific TEAD1 overexpression drives satellite cell hyperplasia and counters pathological effects of dystrophin deficiency. Elife. 2016 Oct 11;5:e15461. doi: 10.7554/eLife.15461.

$$
* * * * *
$$

\#48. May 28, 2021

The ups and downs of stemness - the roles for basal and apical components in satellite cell function

Chen-Ming Fan,* Anwen Shao ${ }^{+}$, Nathalie Gerassimov ${ }^{+}$

Department of Embryology, Carnegie Institution for Science, Baltimore, MD, USA.

* Chen-Ming Fan: fan@carnegiescience.edu

+ These two authors contribute equally

Skeletal muscle stem cells, i.e. satellite cells (SCs), engage in apical contacts with the sarcolemma and basal contacts with the surrounding basement membrane. Protein components that regulate cell polarity or mediate these contacts are known to contribute to SC's quiescence, activation, proliferation, renewal, as well as differentiation. ${ }^{1-3}$ Our understanding of how these components mediate multiple aspects of SC biology remains incomplete. We have previously shown that a tight-junction-like complex (including membrane palmitoylated protein 7, i.e. Mpp7, and Angiomotin ${ }^{2,4}$ at the apical contact and the $\beta 1$-integrin at the basal contact, ${ }^{1}$ mediate multiple aspects of SC function. Extending these lines of investigation, we focus on events downstream of integrins and Mpp7. We will report phenotypes for SC-specific conditional knockouts (SC-cKO) of integrin linked kinase (Ilk), focal adhesion kinase (Fak), ${ }^{5} \mathrm{Mpp} 7$, and Angiomotin in the mouse. For example, single SC-cKOs of Ilk and Fak, two main downstream effectors of integrins, do not phenocopy the SC quiescence-breaking defect reported for $\beta 1$-integrin SC-cKO, but do display defects in subsequent myogenic progression. On the other hand, single SC-cKOs of Mpp7 and Angiomotin show SC proliferation and renewal defects, resulting in severe deficits in regeneration. Further characterization using cell biological, molecular and biochemical approaches is underway to decipher how these components operate in select myo-regenerative steps. Our eventual goal is to reach a comprehensive understanding of the cross-talk between apical and basal components in the SC to orchestrate the step-wise regenerative process of the skeletal muscle in normal, pathological and aging contexts.

Keywords: satellite cell; apical; basal; regeneration.

References

1. Rozo M, Li L, Fan CM. Targeting $\beta 1$-integrin signaling enhances regeneration in aged and dystrophic muscle in mice. Nat Med. 2016 Aug;22(8):889-96. doi: 10.1038/nm.4116. Epub 2016 Jul 4.

2 Li L, Fan CM. ACREB-MPP7-AMOT regulatory axis controls muscle stem cell expansion and self-renewal competence. (2017). Cell Rep. 2017 Oct 31;21(5):1253-1266. doi: 10.1016/j.celrep.2017.10.031.

3. Goel AJ, Rieder MK, Arnold HH, Radice GL, Krauss RS. Niche cadherins control the quiescence-toactivation transition in muscle stem cells. Cell Rep. 2017 Nov 21;21(8):2236-2250. doi: 10.1016/j.celrep. 2017.10.102.

4. Wells CD, Fawcett JP, Traweger A, Yamanaka Y, Goudreault M, Elder K, Kulkarni S, Gish G, Virag C, Lim C, Colwill K, Starostine A, Metalnikov $P$, Pawson T.A Rich1/Amot complex regulates the Cdc42 GTPase and apical-polarity proteins in epithelial cells. Cell. 2006 May 5;125(3):535-48. doi: 10.1016/j.cell. 2006.02.045.

5. Quach NL, Biressi S, Reichardt LF, Keller C, Rando TA. Focal adhesion kinase signaling regulates the expression of caveolin 3 and $\beta 1$ Integrin, genes essential for normal myoblast fusion. Mol Biol Cell. 2009 Jul;20(14):3422-35. doi: 10.1091/mbc.e09-020175. Epub 2009 May 20.

$$
* * * * *
$$

\#49. May 28, 2021

Myogenic renewal capacity of satellite cells measured by Nestin-GFP reporter activity

Kenneth Day (1), Zipora Yablonka-Reuveni (2)

(1) Zymo Research Corp., Irvine, CA USA; University of Washington, Seattle, USA

* Kennet Day: kday@zymoresearch.com

Satellite cells are adult stem cells that are required for adult skeletal muscle regeneration, and contribute to a self-renewed, quiescent reserve population. Somitederived satellite cell populations that reside in anatomically and functionally distinct skeletal muscles differ in their abilities to self-renew, and this process also varies with age. We use the nestin-GFP transgenic mouse model for sorting and tracking of both satellite cell populations and their respective reserve progeny based on GFP reporter expression. Compared to hind limb muscle, sorted GFP+ satellite cell populations isolated from diaphragm produced greater than 3-fold the abundance of nestin-GFP+ reserve cells. Reserve cell 
generation was less impacted by age based on clonal analysis of resident satellite cells from the diaphragm. Quantitative expression analysis revealed $\sim 16$-fold higher Pax3 transcript levels in diaphragm satellite cells compared to limb. These higher relative Pax3 levels were maintained in cultured progeny of diaphragm satellite cells and in sorted reserve cells in primary culture. Alternatively spliced Pax3 mRNAs were also detected in diaphragm satellite cells and reserve progeny, but Pax3 protein was not detectable within diaphragm in vivo or in primary myogenic cultures established from diaphragm. These molecular attributes indicate that satellite cells retain their identity within their muscle origin, but share convergent myogenic programs. Nestin-GFP reporter as an expressed marker of reserve cell development in myogenic culture presents opportunities to investigate mechanistically the influences of cis and trans regulatory elements, epigenetic control and aging using single cell genomics approaches. The unique expression of the reporter in quiescent cells also allows investigation of the local signals in the myogenic niche that maintain or release satellite cells and reserve progeny from the quiescent state in vivo and in co-culture models.

Keywords: nestin-GFP; aging; satellite cells; Pax3; quiescence.

\section{References}

1. Day K, Shefer G, Richardson JB, Enikolopov G, Yablonka-Reuveni Z. Nestin-GFP reporter expression defines the quiescent state of skeletal muscle satellite cells. Dev Biol. 2007 Apr 1;304(1):246-59. doi: 10.1016/j.ydbio.2006.12.026.

2. Day K, Shefer G, Shearer A, Yablonka-Reuveni Z. The depletion of skeletal muscle satellite cells with age is concomitant with reduced capacity of single progenitors to produce reserve progeny. Dev Biol. 2010 Apr 15;340(2):330-43. doi: 10.1016/j.ydbio. 2010.01.006.

3. Stuelsatz P, Keire P, Yablonka-Reuveni Z. Isolation, Culture, and Immunostaining of Skeletal Muscle Myofibers from Wildtype and Nestin-GFP Mice as a Means to Analyze Satellite Cell. Methods Mol Biol. 2017;1556:51-102. doi: 10.1007/978-1-4939-67711 1_.

4. Stuelsatz P, Shearer A, Li Y, Muir LA, Ieronimakis N, Shen QW, Kirillova I, Yablonka-Reuveni Z. Extraocular muscle satellite cells are high performance myo-engines retaining efficient regenerative capacity in dystrophin deficiency. Dev Biol. 2015 Jan 1;397(1):31-44. doi: 10.1016/j.ydbio.2014.08.035.
\#50. May 28, 2021

Molecular and functional diversity among satellite cell populations

\section{Shahragim Tajbakhsh}

Stem Cells \& Development Unit, UMR CNRS 3738, Institut Pasteur, Paris

* Shahragim Tajbakhsh:

shahragim.tajbakhsh@pasteur.fr

Muscle stem cells are heterogeneous in properties, largely resulting from their anatomical location; those in the head are molecularly and functionally distinct from those in the body. Therefore, the modular design that establishes muscles during development might explain why only subsets of muscles exhibit pathology in different myopathies, whereas others escape the disease process. The regulation of skeletal muscle stem cells during homeostasis and regeneration involves an interplay between extrinsic and intrinsic cues. Multiple pathways have been identified that modulate muscle stem cell quiescence and properties. Notably, the proliferation and differentiation properties of extraocular and limb muscles are distinct. To address the underlying mechanisms of this diversity, we performed transcriptional profiling and functional assays of satellite cells isolated from cranial and limb muscles and identified core gene regulatory networks that are common and unique to each population. How extracellular signaling impinges on intracellular decisions to differentially regulate proliferation and differentiation in vivo and ex vivo will be discussed.

Keywords: muscle stem cell heterogeneity; cranial myogenesis; Myogenin-Tomato KI mouse.

\section{References}

1. Benavente-Diaz M, Comai G, Di Girolamo D, Langa $F$, Tajbakhsh S. Dynamics of myogenic differentiation using a novel Myogenin knock-in reporter mouse. Skelet Muscle. 2021 Feb 18;11(1):5. doi: 10.1186/s13395-021-00260-X.

2. Comai GE, Tesařová $M$, Dupé $V$, Rhinn $M$, Vallecillo-García P, da Silva F, Feret B, Exelby K, Dollé P, Carlsson L, Pryce B, Spitz F, Stricker S, Zikmund T, Kaiser J, Briscoe J, Schedl A, Ghyselinck NB, Schweitzer R, Tajbakhsh S. Local retinoic acid signaling directs emergence of the extraocular muscle functional unit. PLoS Biol. $2020 \mathrm{Nov}$ 17;18(11):e3000902. 10.1371/journal.pbio.3000902.

3. Evano E\#*, Gill D\#, Hernando-Herraez H\#, Comai $G$, Stubbs T, Commere PH, Reik W*, Tajbakhsh $S^{*}$. Transcriptome and epigenome diversity and plasticity of muscle stem cells following transplantation. doi: 10.1101/2020.05.20.107219 \#co-first authors; *co-corresponding. PLOS 
Genetics, Oct 2020;30;16(10):e1009022. doi: 10.1371/ journal.pgen.1009022. eCollection 2020.

4. Evano B, Khalilian S, Le Carrou G, Almouzni G, Tajbakhsh S. Dynamics of Asymmetric and Symmetric Divisions of Muscle Stem Cells In Vivo and on Artificial Niches. Cell Rep. 2020 Mar 10;30(10):3195-3206.e7. doi: 10.1016/j.celrep. 2020.01.097.

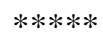

\#51. May 28, 2021

Insights into the epigenetic regulation of myogenesis from an unlikely source

Michael P. Phelps (1),* Eleanor Chen (2)

(1) Department of Animal Sciences, Washington State University, Pullman, WA, USA; (2) Department of Pathology, University of Washington, Seattle, WA USA.

* Michael Phelps: michael.phelps1@wsu.edu

While myogenic differentiation is known to be driven by the coordinated expression of myogenic regulatory factors (MRFs) how these factors are regulated throughout myogenesis is not well understood. Using human rhabdomyosarcoma cancer cells as an unlikely model system, we discovered that myogenic differentiation is regulated by histone deacetylase (HDAC) epigenetic reprogramming that suppresses myogenic gene expression. Rhabdomyosarcoma is a pediatric cancer that is characterized by a sustained myoblast-like proliferative state. Disruption of the oncogenic drivers of diverse rhabdomyosarcoma cancer cells leads to largescale myogenic differentiation suggesting that the cancer cells retain their myogenic capacity. Using a CRISPR arrayed screening approach to investigate the function of all HDAC Class I genes in rhabdomyosarcoma, HDAC3 was identified as a major repressor of myogenesis in these cancer cells. Structurefunction studies revealed that HDAC3 works within the nuclear receptor corepressor complex (NCOR) to prevent the activation of myogenesis. Targeted ablation of members of the HDAC3/NCOR complex resulted in complete myogenic differentiation of the cancer cells, which can be rescued through overexpression of functional genes. We have established a working model for the potential role of the HDAC3/NCOR complex in regulating MRF function during myogenesis.

Keywords: Rhabdomyosarcoma; Histone Deacetylase; CRISPR; Epigenetics; Differentiation.

\section{References}

1. Phelps MP, Bailey JN, Vleeshouwer-Neumann T, Chen EY. CRISPR screen identifies the NCOR/HDAC3 complex as a major suppressor of differentiation in rhabdomyosarcoma. Proc Natl
Acad Sci U S A. 2016 Dec 27;113(52):15090-15095. doi: 10.1073/pnas.1610270114. Epub 2016 Dec 12..

2. Phelps MP, Yang $H$, Patel S, Rahman MM, McFadden G, Chen E. Oncolytic Virus-Mediated RAS Targeting in Rhabdomyosarcoma. Mol Ther Oncolytics. 2018 Sep 15;11:52-61. doi: 10.1016/j.omto.2018.09.001..

3. Vleeshouwer-Neumann T, Phelps M, Bammler TK, MacDonald JW, Jenkins I, Chen EY. Histone Deacetylase Inhibitors Antagonize Distinct Pathways to Suppress Tumorigenesis of Embryonal Rhabdomyosarcoma. PLoS One. 2015 Dec 4;10(12):e0144320. doi: 10.1371/journal.pone. 0144320 .

\#52. May 28, 2021

Satellite cell dysfunction in facioscapulohumeral muscular dystrophy

Massimo Ganassi, Christopher R. S. Banerji, Philipp Heher, Elise Engquist, Johanna Prüller, Peter S. Zammit

King's College London, Randall Centre for Cell and Molecular Biophysics, New Hunt's House, Guy's Campus, London, UK

* Peter S. Zammit: peter.zammit@kcl.ac.uk

Facioscapulohumeral muscular dystrophy (FSHD) is a prevalent autosomal-dominant myopathy, characterized by a slowly progressive skeletal muscle weakness and wasting. We recently found that a regenerative response is elicited in FSHD muscle (Banerji et al., 2020). Muscle biopsies from FSHD patients have a transcriptomic signature characteristic of muscle regeneration, with an average of $0.5 \%$ regenerating myofibers per quadriceps biopsy, rising to $1.7 \%$ for tibialis anterior. Muscle regeneration in FSHD is correlated with pathology, as marked by central nucleation of muscle fibers, fibrosis and necrosis. To investigate regenerative myogenesis in FSHD, we have performed extensive analysis of RNASeq data. We found that FSHD is characterised by suppression of PAX7 target genes (Banerji et al., 2017) ${ }^{2}$ and identified several pathways that are perturbed (e.g. Banerji et al., 2019). ${ }^{3}$ Here, I will update on our progress in understanding regenerative myogenesis in FSHD.

Keywords: facioscapulohumeral muscular dystrophy; muscle regeneration; satellite cell.

References

1. Banerji CRS, Henderson D, Tawil RN, Zammit PS. Skeletal muscle regeneration in facioscapulohumeral muscular dystrophy is correlated with pathological severity. Hum Mol Genet. 2020 Sep 29;29(16):27462760. doi: $10.1093 / \mathrm{hmg} /$ ddaa164.

2 Banerji CRS, Panamarova M, Hebaishi H, White RB, Relaix F, Severini S, Zammit PS. PAX7 target genes are globally repressed in facioscapulohumeral 
muscular dystrophy skeletal muscle. Nat Commun. 2017 Dec18;8(1):2152. doi: 10.1038/s41467-017-01200-4.

3. Banerji CRS, Panamarova M, Pruller J, Figeac N, Hebaishi H, Fidanis E, Saxena A, Contet J, Sacconi $S$, Severini S, Zammit PS. Dynamic transcriptomic analysis reveals suppression of PGC1 $\alpha / E R R \alpha$ drives perturbed myogenesis in facioscapulohumeral muscular dystrophy. Hum Mol Genet. 2019 Apr 15;28(8):1244-1259. doi: 10.1093/hmg/ddy405.

$$
* * * * *
$$

\#53. May 28, 2021

\section{When skeletal muscle turns to bone: pathology, progenitors, and therapeutics}

Sean J. Stoessel, Lorraine N. Apuzzo, Amanda Harrop, Masakazu Yamamoto, David J. Goldhamer*

Department of Molecular \& Cell Biology, University of Connecticut Stem Cell Institute, University of Connecticut, Storrs, CT, USA

* David J. Goldhamer: david.goldhamer@uconn.edu

Heterotopic ossification (HO), the formation of bone in skeletal muscles and associated soft tissues, is manifested in its most extreme form in the rare genetic disease, fibrodysplasia ossificans progressiva (FOP), which is caused by a gain-of-function mutation in the BMP type I receptor, ACVR1 [ACVR1(R206H)]. FOP patients suffer progressive and life-long severe disability as a cumulative consequence of broadly distributed $\mathrm{HO}$, which interferes with skeletal muscle function, and results in joint ankylosis and other complications that dramatically decrease quality of life and life expectancy. We have shown that fibro/adipogenic progenitors (FAPs) are a major contributor to $\mathrm{HO}$ in an accurate genetic mouse model of FOP. Using conditional mutagenesis and receptor over-expression, we show that the stoichiometric balance of wild type (WT) to mutant ACVR1 receptors is a key determinant of disease severity, consistent with a model in which WT and mutant ACVR1 compete for limiting ligand or type II receptor dimerization partners. We have undertaken transcriptome analyses of FAPs during critical early stages of disease progression to better understand molecular underpinnings of their pathogenic commitment to skeletogenic fates resulting from dysregulated signaling through ACVR1(R206H). Key regulators of chondrogenesis and osteogenesis, including Sox9, Runx2, and Osterix, were expressed by 3 days post-injury, 2 to 3 days prior to the histological appearance of cartilage, and 4 to 7 days prior to detection of bone by microCT and histology. Other gene expression changes will be discussed. Previous studies have identified activin $\mathrm{A}$ as an essential ligand for ACVR1(R206H)-dependent HO in FOP mice. We show that treatment of FOP mice with an anti-activin A antibody normalizes the FAP transcriptome at early postinjury time points, indicating that activin A-dependent pathogenic processes include pre-skeletal stages, consistent with the requirement for activin A in the early expansion of FOP FAPs in a transplantation model. These studies implicate FAPs as a central therapeutic target for the treatment of FOP.

Keywords: FOP; heterotopic ossification; fibro/ adipogenic progenitors; activin A.

\section{References}

1. Hatsell SJ, Idone V, Wolken DM, Huang L, Kim HJ, Wang L, Wen X, Nannuru KC, Jimenez J, Xie L, Das $N$, Makhoul G, Chernomorsky R, D'Ambrosio D, Corpina RA, Schoenherr CJ, Feeley $K, Y u P B$, Yancopoulos GD, Murphy AJ, Economides AN. ACVR1R206H receptor mutation causes fibrodysplasia ossificans progressiva by imparting responsiveness to activin A. Sci Transl Med. 2015 Sep 2;7(303):303ra137. doi: 10.1126/scitranslmed.aac4358.

2. Hino K, Ikeya M, Horigome K, Matsumoto $Y$, Ebise $H$, Nishio $M$, Sekiguchi $K$, Shibata M, Nagata S, Matsuda S, Toguchida J. Neofunction of ACVR1 in fibrodysplasia ossificans progressiva. Proc Natl Acad Sci U S A. 2015 Dec 15;112(50):15438-43. doi: 10.1073/pnas.1510540112. Epub 2015 Nov 30.

3. Lees-Shepard JB, Yamamoto M, Biswas AA, Stoessel SJ, Nicholas SE, Cogswell CA, Devarakonda PM, Schneider MJ Jr, Cummins SM, Legendre NP, Yamamoto S, Kaartinen V, Hunter JW, Goldhamer DJ. Activin-dependent signaling in fibro/adipogenic progenitors causes fibrodysplasia ossificans progressiva. Nat Commun. 2018 Feb 2;9(1):471. doi: 10.1038/s41467-018-02872-2.

4. Lees-Shepard JB, Nicholas SE, Stoessel SJ, Devarakonda PM, Schneider MJ, Yamamoto M, Goldhamer DJ. Palovarotene reduces heterotopic ossification in juvenile FOP mice but exhibits pronounced skeletal toxicity. Elife. 2018 Sep 18;7:e40814. doi: 10.7554/eLife.40814.

5. Shore EM, Xu M, Feldman GJ, Fenstermacher DA, Cho TJ, Choi IH, Connor JM, Delai P, Glaser DL, LeMerrer M, Morhart R, Rogers JG, Smith R, Triffitt JT, Urtizberea JA, Zasloff M, Brown MA, Kaplan FS. A recurrent mutation in the BMP type I receptor ACVR1 causes inherited and sporadic fibrodysplasia ossificans progressiva. Nat Genet. 2006 May; 38(5):525-7. doi: 10.1038/ng1783. Epub 2006 Apr 23.

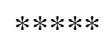

\#54. May 28, 2021

\section{Cellular dynamics of muscle regeneration}

Brittany C Collins, Jacob B Shapiro, Robert V Musci, Mya Scheib, Gabrielle Kardon

Department of Human Genetics, University of Utah, Salt Lake City, UT, USA

* Gabrielle Kardon: gkardon@genetics.utah.edu 
Vertebrate skeletal muscle is composed of post-mitotic, multinucleate myofibers. In response to injury, skeletal has a remarkable capacity to regenerate. A population of dedicated stem cells, the satellite cells, is responsible for regenerating the myofibers. In the absence of injury, satellite cells are quiescent and reside in a niche between the plasmalemma and the basement of the mature myofibers. However, injury stimulates satellite cells to activate, proliferate, migrate, fuse, and differentiate and regenerate damaged myofibers. Interactions between myogenic cells, macrophages and endothelial cells are also critical to the regenerative process. There have been limited efforts to visualize the in vivo cellular response to injury largely due to the difficulty of imaging skeletal muscle in three dimensions. We use novel techniques to image muscle regeneration in whole-mount, in combination with genetic lineage to label and track the fate of satellite cells and immunofluorescence to label endothelial cells and macrophages. Our studies provide critical new insights into the cellular dynamics and interactions governing satellite cell-mediated regeneration.

Keywords: muscle regeneration; satellite cells; macrophages; endothelial cells.

\section{References}

1. Mauro, A. 1961. Satellite cell of skeletal muscle fibers. $J$ Biophys Biochem Cytol. 1961 Feb;9(2):493-5. doi: 10.1083/jcb.9.2.493.

2. Massimino ML, Rapizzi E, Cantini $M$, Libera LD, Mazzoleni F, Arslan P, Carraro U. ED2+ macrophages increase selectively myoblast proliferation in muscle cultures. Biochem Biophys Res Commun. 1997 Jun 27;235(3):754-9. doi: 10.1006/bbrc.1997.6823.

3. Murphy MM, Lawson JA, Mathew SJ, Hutcheson DA, Kardon G. 2011. Satellite cells, connective tissue fibroblasts, and their interactions are crucial for muscle regeneration. Development. 2011 Sep;138(17):3625-37. doi: 10.1242/dev.064162.

4. Verma M, Murkonda BS, Asakura Y, Asakura A. 2016. Skeletal Muscle Tissue Clearing for LacZ and Fluorescent Reporters, and Immunofluorescence Staining. Methods Mol Biol. 2016;1460:129-40. doi: 10.1007/978-1-4939-38100_10.

5. Tidball, J. G. 2017. Regulation of muscle growth and regeneration by the immune system. Nat Rev Immunol. 2017 Mar;17(3):165-178. doi: 10.1038/nri.2016.150. Epub $2017 \mathrm{Feb} 6$.

\#55. May 28, 2021

\section{Satellite cells provide a critical source of IGF-I during skeletal muscle growth and regeneration}

\section{Hui Jean Kok, Elisabeth R Barton}

\section{University of Florida, FL, USA}

* Elisabeth R. Barton: erbarton@ufl.edu

Satellite cells are a population of muscle stem cells that are crucial for supplying additional myonuclei to muscle fibers during post-natal growth and for repair post-injury. Insulin-like Growth Factor-I (IGF-I) expedites skeletal muscle growth and regeneration by amplifying satellite cell proliferation and accelerating myoblast differentiation. It has been postulated that IGF-I diffuses into the satellite cell niche, however, it remains unclear if satellite cells rely on an autocrine source of IGF-I for their actions, or a paracrine source of IGF-I from the myofibers or other cell types. Thus, we sought to determine the critical sources of IGF-I for muscle growth and regeneration. We generated novel mouse models with inducible tissue- and cell-specific ablation of IGF-I: MID (Muscle IGF-I Deficient) ${ }^{1}$ SID (ㅁatellite cell IGF-I Deficient), SMID (ㅁatellite cell and Muscle IGF-I Deficient) and CTRL (control mice lacking the floxed exon 4 of Igf1), to test the hypothesis that satellite cells require an autocrine source of IGF-I for muscle regeneration. To assess the efficiency of muscle regeneration, mice ( $n=4 /$ sex/timepoint/genotype) were treated with Tamoxifen (TAM) and/or Doxycycline (DOX) to induce IGF-I deletion prior to unilateral Cardiotoxin (CTX) injection, targeting the tibialis anterior (TA) as previously described. ${ }^{2}$ The mass of TA muscles from all groups at days 3-7 post-CTX were significantly lower $(\mathrm{p}<0.05)$ than contralateral nondamaged muscles. All groups were able to recover to non-damaged mass levels at day 14 post-CTX except SID animals. Importantly, muscles from SID and SMID mice displayed $\sim 36 \%$ decreases in fiber sizes compared to CTRL and MID at day 7 post-CTX using SMASH analysis, ${ }^{3}$ suggesting the importance of IGF-I from satellite cells for muscle regeneration. Surprisingly, SMID mice were able to recover to CTRL levels in fiber area, indicating a potential compensatory IGF-I supply from non-muscle sources. Next, we tested the hypothesis that satellite cell IGF-I is also critical for muscle development. Pups at post-natal day 3 (P3) were treated with TAM and/or DOX for 5 days to induce Igf 1 deletion, and muscles were harvested at P28. Additional vehicle (VEH) treated pups were used as littermate controls. We observed a $25 \%$ and $30 \%$ decrease in quadriceps mass of SID and SMID animals, respectively, when compared to CTRLs. This points to the importance of muscle IGF-I content for muscle development. In conclusion, IGF-I generated by satellite cells is critical for efficient skeletal muscle regeneration, while both satellite cells and myofibers provide integral sources of IGF-I for muscle development.

Keywords: Satellite cells; IGF-I; regeneration; autocrine signaling.

\section{References}

1. Vassilakos G, Lei H, Yang Y, Puglise J, Matheny M, Durzynska J, Ozery $M$, Bennett $K$, Spradlin $R$, Bonanno H, Park S, Ahima RS, and Barton ER. Deletion of muscle IGF-I transiently impairs growth and progressively disrupts glucose homeostasis in 
male mice. FASEB J 2019 Jan;33(1):181-194. doi: 10.1096/fj.201800459R. Epub 2018 Jun 22..

2. Smith LR, Kok HJ, Zhang B, Chung D, Spradlin RA, Rakoczy KD, Lei H, Boesze-Battaglia K, Barton ER. Matrix Metalloproteinase 13 from Satellite Cells is Required for Efficient Muscle Growth and Regeneration. Cell Physiol Biochem. 2020 Apr 11;54(3):333-353. doi: 10.33594/000000223.

3. Smith LR, Barton ER. SMASH - semi-automatic muscle analysis using segmentation of histology: a MATLAB application. Skelet Muscle. 2014 Nov 27;4:21. doi: 10.1186/2044-5040-4-21.

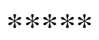

\#56. May 28, 2021 A nutritional approach to promote muscle
regeneration by targeting Muscle Stem Cells

Sara Ancel $(1,2)$, Joris Michaud (1), Jerome Feige $(1,2)$, Pascal Stuelsatz (1)

(1) Nestlé Research, EPFL Innovation Park, Lausanne, Switzerland; (2) School of Life Sciences, Ecole Polytechnique Fédérale de Lausanne (EPFL), Switzerland

* Pascal Stuelsatz: pascal.stuelsatz@rd.nestle.com

Skeletal muscle regeneration is a crucial mechanism to repair and maintain muscle mass and function throughout life. Skeletal muscle regeneration primarily requires the participation of myogenic progenitors, termed muscle stem cells (MuSCs). In a number of pathological conditions that lack effective therapeutic interventions, such as sarcopenia, muscular dystrophies, or cachexia, skeletal muscles show a decline in MuSCs function and a reduced repair ability. ${ }^{1-4}$ MuSC function has also been associated with muscle adaptation to every day physical activity, playing a role in muscle adaptation in response to exercise. ${ }^{5-7}$ Muscle stem cell function and regeneration can be promoted/accelerated both in healthy and diseased conditions to enhance muscle repair and maintain muscle function. Therapeutic modulation of MuSC function has mainly been investigated using pharmacologic drugs but interest for nutritional interventions has been recently growing. ${ }^{8-10}$ In an effort to identify natural bioactive that could promote MuSC regenerative capacity, we have developed and performed an in vitro high content screening assay. Out of this screen, we selected few frontrunners that we have tested in an in vivo model of muscle regeneration. These preclinical studies have successfully demonstrated the ability of these compounds to promote MuSC activity and muscle repair.

Keywords: muscle stem cells; nutritional intervention; muscle regeneration and repair.

\section{References}

1. He WA, Berardi E, Cardillo VM, Acharyya S, Aulino $P$, Thomas-Ahner J, Wang J, Bloomston $M$,
Muscarella P, Nau P, Shah N, Butchbach ME, Ladner K, Adamo S, Rudnicki MA, Keller C, Coletti D, Montanaro F, Guttridge DC.NF-kappaB-mediated Pax7 dysregulation in the muscle microenvironment promotes cancer cachexia. J Clin Invest. 2013 Nov;123(11):4821-35. doi: 10.1172/JCI68523.

2. Tierney MT, Stec MJ, Rulands S, Simons BD, Sacco A. Muscle Stem Cells Exhibit Distinct Clonal Dynamics in Response to Tissue Repair and Homeostatic Aging. Cell Stem Cell. 2018 Jan 4;22(1):119-127.e3. 10.1016/j.stem.2017.11.009. Epub 2017 Dec 14.

3. Blau HM, Cosgrove BD, Ho AT. The central role of muscle stem cells in regenerative failure with aging. Nat Med. 2015 Aug;21(8):854-62. doi: 10.1038/nm.3918..

4. Dumont NA, Wang YX, von Maltzahn J, Pasut A, Bentzinger CF, Brun CE, Rudnicki MA. Dystrophin expression in muscle stem cells regulates their polarity and asymmetric division. Nat Med. 2015 Dec;21(12):1455-63. doi: 10.1038/nm.3990. Epub 2015 Nov 16.

5. Englund DA, Murach KA, Dungan CM, Figueiredo VC, Vechetti IJ Jr, Dupont-Versteegden EE, McCarthy JJ, Peterson CA. Depletion of resident muscle stem cells negatively impacts running volume, physical function, and muscle fiber hypertrophy in response to lifelong physical activity. Am J Physiol Cell Physiol. 2020 Jun 1;318(6):C1178-C1188. doi: 10.1152/ajpcell.00090.2020. Epub 2020.

6. Masschelein E, D'Hulst G, Zvick J, Hinte L, SoroArnaiz I, Gorski T, von Meyenn F, Bar-Nur O, De Bock K. Exercise promotes satellite cell contribution to myofibers in a load-dependent manner. Skelet Muscle. 2020 Jul 9;10(1):21. doi: 10.1186/s13395020-00237-2.

7. Davis A Englund, Vandré C Figueiredo, Cory $M$ Dungan, Kevin A Murach, Bailey D Peck, Jennifer M Petrosino, Camille R Brightwell, Alec M Dupont, Ally C Neal, Christopher S Fry, Federica Accornero, John J McCarthy, Charlotte A Peterson. Satellite Cell Depletion Disrupts Transcriptional coordination and muscle adaptation to Exercise. Function 2021; 2(1).

8. Woo $M$, Isganaitis E, Cerletti $M$, Fitzpatrick $C$, Wagers AJ, Jimenez-Chillaron J, Patti ME. Early life nutrition modulates muscle stem cell number: implications for muscle mass and repair. Stem Cells Dev. 2011 Oct;20(10):1763-9. doi: 10.1089/scd. 2010.0349. Epub 2011 Mar 9.

9. Zhang H, Ryu D, Wu Y, Gariani K, Wang $X$, Luan $P$, D'Amico D, Ropelle ER, Lutolf MP, Aebersold R, Schoonjans K, Menzies KJ, Auwerx J. NAD(+) repletion improves mitochondrial and stem cell function and enhances life span in mice. Science. 2016 Jun 17;352(6292):1436-43. doi: 10.1126/ science.aaf2693. Epub 2016 Apr 28.

10. Nierobisz LS, Cheatham B, Buehrer BM, Sexton JZ. High-content screening of human primary muscle 
satellite cells for new therapies for muscular atrophy/dystrophy. Curr Chem Genom Transl Med. 2013 Sep 3;7:21-9. doi: 10.2174/221398850130 7010021. eCollection 2013.

$* * * * *$

\#57. May 28, 2021

\section{AAV-micro-dystrophin gene therapy for Duchenne} Muscular Dystrophy

H. Lee Sweeney, David Hammers, Matt Lee, Cora Coker, and Michael Matheny

\section{Myology Institute, University of Florida, USA}

*H. Lee Sweeney: 1sweeney@ufl.edu

Duchenne muscular dystrophy (DMD) is a caused by loss of the force transmitting and membrane complex organizing protein, dystrophin, and is characterized by progressive skeletal muscle deterioration with failed regeneration and replacement with a fatty-fibrous matrix1. The disease affects both cardiac and skeletal muscles, with the heart undergoing a dilated cardiomyopathy2. Death is due to a combination of respiratory and cardiac muscle failure. Micro-dystrophin gene therapy has entered clinical trials and is now poised to be a transformative therapy for the disease. However, the impact of this gene therapy in humans is unknown, and most of the animal studies with these vectors have been performed in the mdx mouse on the C57/Bl10 background, suffers from being a mild phenotype. We used a severe mouse model of DMD, the mdx mouse on the DBA background (D2.mdx mouse), which has heightened inflammation and fibrosis as compared to the more widely utilized $\mathrm{mdx}$ mouse on the C57/Bl10 background. We administered AAV micro-dystrophin gene therapy, using micro-dystrophins that are similar to those currently in clinical trials, at one month or three months of age. At 6 months of age (sedentary), skeletal muscles demonstrated functional rescue, with greatly diminished fibrosis. However, by 12 months of age, there was significant disease progression. Thus it appears that the micro-dystrophins can modify disease progression in skeletal muscle, but that they do no stop progression. We are currently examining combination therapies with micro-dystrophin to attempt to further block disease progression and to delineate the deficits of the microdystrophin constructs in clinical trials.

Keywords: AAV-micro-dystrophin; gene therapy; Duchenne Muscular Dystrophy.

\section{References}

1. Forbes SC, Willcocks RJ, Triplett WT, Rooney WD, Lott DJ, Wang DJ, Pollaro J, Senesac CR, Daniels MJ, Finkel RS, Russman BS, Byrne BJ, Finanger EL, Tennekoon GI, Walter GA, Sweeney HL, Vandenborne K. Magnetic resonance imaging and spectroscopy assessment of lower extremity skeletal muscles in boys with Duchenne muscular dystrophy: a multicenter cross sectional study. PLoS One. 2014 Sep 9;9(9):e106435. doi: 10.1371/journal.pone. 0106435. eCollection 2014.

$$
* * * * *
$$

\#58. May 28, 2021

Therapeutic approaches for oculopharyngeal muscular dystrophy: where are we now?

Capucine Trollet, Gillian Butler-Browne

Sorbonne Université, Inserm, Institut de Myologie, Centre de Recherche en Myologie, Paris, France.

* Capucine Trollet: capucine.trollet@upmc.fr

Oculopharyngeal muscular dystrophy (OPMD) is a rare and slow-progressing, late-onset, autosomal dominant inherited neuromuscular disorder that starts from midlife onwards and progresses with age. It is characterized by progressive ptosis, dysphagia and proximal limb weakness. Despite the weakness associated with the other muscle groups, it is the complications of dysphagia (choking, regurgitation, aspiration pneumonia, poor nutrition) that most often requires serious intervention in order to preserve life. OPMD is caused by a short (GCN)1-8 expansion in the gene encoding polyadenylate RNA binding protein nuclear 1 (PABPN1). ${ }^{1}$ The mutation results in an expanded $\mathrm{N}$-terminal polyalanine tract and the misfolded protein (expPABPN1) forms nuclear insoluble ribonucleoproteic aggregates in muscle fibers. ${ }^{2,3}$ Although the physiopathological mechanisms involved in OPMD are still under investigations, therapeutic strategies are being developed in parallel, since the mutation is known and restricted muscles are affected. Currently OPMD patients can only be referred to surgeons for cricopharyngeal myotomy or corrective surgery to extraocular muscles to ease ptosis. Over the last years we have been working on pharmacological, cell-based and gene-based therapies for OPMD. Pharmacological approach consists in testing antiaggregation drugs to remove intranuclear PABPN1 aggregates. ${ }^{4,5}$ Cell-based therapy consists in transplanting autologous myoblasts to improve affected pharyngeal muscle function. ${ }^{6}$ Gene therapy approach consists in using adeno-associated viral vector (AAV) since the size of PABPN1 allows it: the approach is based on a local AAV delivery in the pharyngeal muscles to both silence mutated PABPN1 and bring back a modified but functional PABPN1 protein in muscle fibers. ${ }^{4,5}$ An overview and update on these strategies will be given.

Keywords: Oculopharyngeal Muscular Dystrophy; aggregation; PABPN1; cell therapy; AAV; gene therapy; dysphagia.

\section{References}

1. Brais B, Bouchard JP, Xie YG, Rochefort DL, Chrétien $N$, Tomé FM, Lafrenière RG, Rommens JM, Uyama E, 
Nohira O, Blumen S, Korczyn AD, Heutink P, Mathieu $J$, Duranceau A, Codère F, Fardeau M, Rouleau GA. Nat Genet. 1998 Feb;18(2):164-7. doi: 10.1038/ng0298-164.

2. Tomé FM, Fardeau M. Nuclear inclusions in oculopharyngeal dystrophy. Acta Neuropathol. 1980;49(1):85-7. doi: 10.1007/BF00692226.

3. Gidaro T, Negroni E, Perié S, Mirabella M, Lainé J, Lacau St Guily J, Butler-Browne G, Mouly V, Trollet C. Atrophy, fibrosis, and increased PAX7-positive cells in pharyngeal muscles of oculopharyngeal muscular dystrophy patients. J Neuropathol Exp Neurol. 2013 Mar;72(3):234-43. doi: 10.1097/NEN.0b013e3182854c07.

4. Bamia A, Sinane M, Naït-Saïdi R, Dhiab J, Keruzoré M, Nguyen PH, Bertho A, Soubigou F, Halliez S, Blondel $M$, Trollet $C$, Simonelig $M$, Friocourt $G$, Béringue V, Bihel F, Voisset C. Anti-prion Drugs Targeting the Protein Folding Activity of the Ribosome Reduce PABPN1 Aggregation. Neurotherapeutics. 2021 Feb 2. doi: 10.1007/s13311-020-00992-6. Epub ahead of print.

5. Malerba A, Roth F, Harish P, Dhiab J, Lu-Nguyen N, Cappellari O, Jarmin S, Mahoudeau A, Ythier V, Lainé $J$, Negroni E, Abgueguen E, Simonelig M, Guedat $P$, Mouly V, Butler-Browne $G$, Voisset $C$, Dickson $G$, Trollet C. Pharmacological modulation of the ER stress response ameliorates oculopharyngeal muscular dystrophy. Hum Mol Genet. 2019 May 15;28(10):16941708. doi: 10.1093/hmg/ddz007.

6. Périé S, Trollet C, Mouly V, Vanneaux V, Mamchaoui K, Bouazza B, Marolleau JP, Laforêt P, Chapon F, Eymard $B$, Butler-Browne G, Larghero J, St Guily JL. Autologous myoblast transplantation for oculopharyngeal muscular dystrophy: a phase I/IIa clinical study. Mol Ther. 2014 Jan;22(1):219-25. doi: 10.1038/mt.2013.155. Epub 2013 Jul 8.

7. Malerba A, Klein P, Bachtarzi H, Jarmin SA, Cordova $G$, Ferry A, Strings V, Espinoza MP, Mamchaoui $K$, Blumen SC, St Guily JL, Mouly V, Graham M, ButlerBrowne G, Suhy DA, Trollet C, Dickson G. PABPN1 gene therapy for oculopharyngeal muscular dystrophy. Nat Commun. 2017 Mar 31;8:14848. doi: 10.1038/ncomms 14848.8 .

8. Malerba A, Klein $P$, Lu-Nguyen $N$, Cappellari $O$, Strings-Ufombah V, Harbaran S, Roelvink P, Suhy D, Trollet C, Dickson G. Established PABPN1 intranuclear inclusions in OPMD muscle can be efficiently reversed by $A A V$-mediated knockdown and replacement of mutant expanded PABPN1. Hum Mol Genet. 2019 Oct 1;28(19):3301-3308. doi: 10.1093/ hmg/ddz167.

$$
* * * * *
$$

\#59. May 28, 2021

Cholesterol metabolism is a potential therapeutic target in Duchenne Muscular Dystrophy

Fatima Amor $(1,2)^{+}$, Ai Vu Hong $(1,2)^{+}$, Guillaume Corre $(1,2)$, Mathilde Sanson (1,2), Laurence Suel $(1,2)$, Stephanie Blaie (1), Laurent Servais (3), Thomas Voit (4), Isabelle Richard (1,2), and David Israeli $(1,2)$ *

+ These authors equally contributed
(1) Généthon, Evry, France; (2) Université ParisSaclay, Univ Evry, Inserm UMR_S951, Evry, France

* David Israeli: israeli@genethon.fr

Duchenne Muscular Dystrophy (DMD) is a lethal muscle disease detected in approximately 1:5000 male births. DMD is caused by mutations in the DMD gene, encoding a critical protein that link the cytoskeleton and the extracellular matrix in skeletal and cardiac muscles. The primary consequence of the disrupted link between the extracellular matrix and the myofiber actin cytoskeleton is involved sarcolemma destabilization, perturbation of $\mathrm{Ca} 2+$ homeostasis, activation of proteases, mitochondrial damage and tissue degeneration. A recently emphasized secondary aspect of the dystrophic process is a progressive metabolic change of the dystrophic tissue; however, the mechanism and nature of the metabolic dysregulation are yet poorly understood. In the present study, ${ }^{1}$ we characterized a molecular mechanism of metabolic perturbation in DMD. We sequenced plasma miRNA in a DMD cohort, comprising of 54 DMD patients treated or not by glucocorticoid, compared to 27 healthy controls, in three age groups. We identified 96 dysregulated miRNAs, of which 74 were up- and 22 down-regulated in DMD. We confirmed the dysregulation in DMD of the Dystro-miRs, Cardio-miRs and a large number of the DLK1-DIO3 miRNAs. ${ }^{2-4} \mathrm{We}$ also identified numerous dysregulated miRNAs, yet unreported in DMD. We then developed an original bioinformatic approach, which is based on miRNAs' both target and host genes, for the biological interpretation of miRNA dysregulation. This bioinformatic analysis predicted that lipid metabolism is highly dysregulated in DMD. Investigation of skeletal muscles of the mdx mouse model revealed dysregulation of transcription factors of cholesterol and fatty acid metabolism (SREBP1 and SREBP2), perturbation of the mevalonate pathway, and accumulation of cholesterol in skeletal muscles. Elevated cholesterol level was also found in muscle biopsies of DMD patients. Treatment of mdx mice with Simvastatin normalized these perturbations and partially restored some dystrophic parameters. This investigation supports that cholesterol metabolism and the mevalonate pathway are therapeutic targets in DMD.

Keywords: Duchenne Muscular dystrophy; host gene; biological interpretation of miRNA dysregulation; SREBP2; lipid metabolism; Cholesterol; Simvastatin

\section{References}

1. Amor F, Vu Hong A, Corre G, Sanson M, Suel L, Blaie $S$ et al. Cholesterol metabolism is a potential therapeutic target in Duchenne Muscular Dystrophy. bioRxiv 2020;2020.12.01.405910.

2. Vignier N, Amor F, Fogel P, Duvallet A, Poupiot J, Charrier S, Arock M, Montus M, Nelson I, Richard I, Carrier L, Servais L, Voit T, Bonne G, Israeli D. Distinctive serum miRNA profile in mouse models of 
striated muscular pathologies. PLoS One. 2013;8(2):e55281. doi: 10.1371/journal.pone.0055 281. Epub 2013 Feb 13.

3. Jeanson-Leh L, Lameth J, Krimi S, Buisset J, Amor F, Le Guiner C, Barthélémy I, Servais L, Blot S, Voit T, Israeli D. Serum Profiling Identifies Novel Muscle miRNA and Cardiomyopathy-Related miRNA Biomarkers in Golden Retriever Muscular Dystrophy Dogs and Duchenne Muscular Dystrophy Patients. Am J Pathol. 2014 Nov;184(11):2885-98. doi: 10.1016/j.ajpath.2014.07.021. Epub 2014 Sep 3.

4. Sanson M, Hog Vu A, Massourides E, Bourg N, Suel $L$, Amor F, Corre G, Bénit P, Barthélémy I, Blot $S$, Bigot A, Pinset C, Rustin P, Servais L, Voit T, Richard I, Israeli D. miR-379 links glucocorticoid treatment with mitochondrial response in Duchenne muscular dystrophy. Sci Rep. 2020 Jun 4;10(1):9139. doi: 10.1038/s41598-020-66016-7.

\#60. May 28, 2021

Comparison of the protein complexes assembled by dystrophin and micro-dystrophin in the heart

Hong Wang (1,2), Elena Marrosu (3), Daniel Brayson (3), Nalinda B. Wasala (4), Eric K. Johnson (1), Charlotte S. Scott (3), Yongping Yue (4), Kwan-Leong Hau (3), Aaron J. Trask (5), Stan C. Froehner (6), Marvin E. Adams (6), Liwen Zhang (7), Dongsheng Duan $(4,8)$, Federica Montanaro $(1,3)^{*}$

(1) Centre for Gene Therapy, The Research Institute at Nationwide Children's Hospital, Columbus, USA; (2) Department of Pediatric Cardiology, Shengjing Hospital, China Medical University, Liaoning, China; (3) Dubowitz Neuromuscular Centre, UCL Great Ormond Street Institute of Child Health, London, UK; (4) Department of Molecular Microbiology and Immunology, School of Medicine, University of Missouri, Columbia, USA; (5) Center for Cardiovascular Research, The Research Institute at Nationwide Children's Hospital and the Department of Pediatrics, The Ohio State University College of Medicine, Columbus, USA; (6) Department of Physiology and Biophysics, University of Washington, Seattle, USA; (7) Mass Spectrometry and Proteomics Facility, Campus Chemical Instrument Center, The Ohio State University, Columbus, USA; (8) Departments of Neurology, of Bioengineering, of Biomedical Sciences, and of Biomedical, Biological \& Chemical Engineering, University of Missouri, Columbia, USA.

* Federica Montanaro: f.montanaro@ucl.ac.uk

$\Delta \mathrm{R} 4-\mathrm{R} 23 / \Delta \mathrm{CT}$ micro-dystrophin ( $\mu$ Dys) is a miniaturized version of dystrophin currently evaluated in a Duchenne muscular dystrophy (DMD) gene therapy trial to treat skeletal and cardiac muscle disease. ${ }^{1}$ In preclinical studies, $\mu$ Dys efficiently rescues cardiac histopathology, but only partially normalizes cardiac function. ${ }^{2}$ To gain insights into factors that may impact the cardiac therapeutic efficacy of $\mu$ Dys, we compared the composition of purified dystrophin and $\mu$ Dys protein complexes in the mouse heart using a proteomics-based approach we previously optimized. ${ }^{3}$ We report that compared to dystrophin, $\mu$ Dys has altered associations with $\alpha 1$ - and $\beta 2$-syntrophins, as well as cavins, a group of caveolae-associated signalling proteins. ${ }^{4}$ In particular, we found that membrane localization of cavins -1 and -4 in cardiomyocytes requires dystrophin and is profoundly disrupted in the heart of $m d x^{5 c v}$ mice, a model of DMD. Under cardiac stress conditions, membrane-associated cavin-4 is known to recruit the signalling molecule ERK to caveolae, which activates key cardio-protective responses. ${ }^{5}$ Evaluation of ERK signalling revealed a profound inhibition, below physiological baseline, in the $m d x^{5 c v}$ mouse heart. Expression of $\mu$ Dys in $m d x^{5 c v}$ mice prevented the development of cardiac histopathology but did not rescue membrane localization of cavins nor did it normalize ERK signalling. Our study provides the first comparative analysis of purified protein complexes assembled in vivo by full-length dystrophin and a therapeutic micro-dystrophin construct. This has revealed disruptions in cavins and ERK signalling, that suggest an impaired cardio-protective response in DMD. This new knowledge is relevant to ongoing efforts to prevent and treat heart disease in DMD patients.

Keywords: Duchenne muscular dystrophy cardiomyopathy; micro-dystrophin; caveolae; ERK signaling; proteomic analysis.

References

1. Duan D. Systemic AAV Micro-dystrophin Gene Therapy for Duchenne Muscular Dystrophy. Mol Ther. 2018 Oct 3;26(10):2337-2356. doi: 10.1016/j.ymthe. 2018.07.011. Epub 2018 Jul 17.

2 Townsend D, Blankinship MJ, Allen JM, Gregorevic $P$, Chamberlain JS, Metzger JM. Systemic administration of micro-dystrophin restores cardiac geometry and prevents dobutamine-induced cardiac pump failure. Mol Ther. 2007 Jun;15(6):1086-92. doi: 10.1038/sj.mt. 6300144. Epub 2007 Apr 17.

3 Johnson EK, Zhang L, Adams ME, Phillips A, Freitas MA, Froehner SC, Green-Church KB, Montanaro F. Proteomic analysis reveals new cardiac-specific dystrophin-associated proteins. PLoS One. 2012; 7(8):e43515. doi: 10.1371/journal.pone. 0043515. Epub 2012 Aug 24.

4 Das M, Das D.K. Caveolae, caveolin, and cavins: potential targets for the treatment of cardiac disease. Ann Med. 2012 Sep;44(6):530-41. doi: 10.3109/ 07853890.2011.577445. Epub 2011 Jun 9.

5 Ogata $T$, Naito $D$, Nakanishi $N$, Hayashi $Y K$, Taniguchi T, Miyagawa K, Hamaoka T, Maruyama $N$, Matoba S, Ikeda K, Yamada H, Oh H, Ueyama T. MURC/Cavin-4 facilitates recruitment of ERK to caveolae and concentric cardiac hypertrophy 
induced by alpha1-adrenergic receptors. Proc Natl Acad Sci U S A. 2014 Mar 11;111(10):3811-6. doi: 10.1073/pnas.131535 9111. Epub 2014 Feb 24.

$$
* * * * *
$$

\#61. May 28, 2021

Elevation of cardiomyocyte 2-deoxy-ATP via gene therapy reduces cardiac dysfunction in Duchenne's Muscular Dystrophy mice

Guy Odom (1), Jeffrey S. Chamberlain (1), Michael Regnier (2)*

\section{University of Washington, Seattle, USA}

* Michael Regnier: mregnier@uw.edu

Duchenne muscular dystrophy (DMD) is one of the most frequent forms of muscular dystrophy and is due to mutations in the dystrophin gene. DMD patients typically die due to cardiac and respiratory muscle failure. Thus maintenance of adequate function in both cardiac and skeletal muscle is critical for optimal DMD therapy. Administration of recombinant adenoassociated viral (rAAV)-mediated micro-dystrophin ( $\mu$ Dys) constructs in mdx mice preceding the onset of cardiomyopathy is cardioprotective, however at a late stage of cardiomyopathy, a full rescue of the dysfunction is not achieved. We have developed a gene therapy approach that improve cardiac contractile function by over-expression of the enzyme ribonucleotide reductase (RNR). RNR converts ADP to 2-deoxy-ADP, which is rapidly converted to 2-deoxy-ATP (dATP) in cells. We have reported that AAV6-RNR increases cardiac function in normal and infarcted rodent and porcine heart, increasing systolic pressure development and the rate of pressure increase and decline $(4,5)$ We have shown that dATP is a myosin activator can replace ATP as the contractile substrate for myosin, inducing structural changes that increase its proximity to actin, binding to actin and acto-myosin crossbridge cycling (2, 3 ). Here (1) we compared the relative therapeutic effect of CK8-driven $\mu$ Dys vs cardiac troponin $\mathrm{T}$ (cTnT)-driven RNR in an advanced-age, DMD cardiomyopathy mouse model. We show a restoration of myocardial workload (via Langendorff perfusion), as indicated by rate pressure product (RPP) for baseline function in $\operatorname{mdx}(4 \mathrm{cv})$ mice of 27 months of age. Although $\mu$ Dys appeared to normalize LV developed pressure in mdx mice, this did not result in a significant increase in RPP. The pressure-volume relationship with increased preload was improved by either treatment. However, only RNR treatment resulted in significant improvements in diastolic functional parameters, returning them to values that were similar to wild-type hearts. Both treatments improved systolic function with high workload challenge, without compromising cardiac reserve. These results suggest that targeted expression of RNR to myocardium can significantly improve contractile performance in an advanced-age DMD cardiomyopathy model, and may have therapeutic implications for DMD patients.

Keywords: Duchenne muscular dystrophy; gene therapy; myosin activator; systolic function; 2-deoxy-ATP.

\section{References}

1. Kolwicz SC Jr, Hall JK, Moussavi-Harami F, Chen $X$, Hauschka SD, Chamberlain JS, Regnier M, Odom GL. Gene Therapy Rescues Cardiac Dysfunction in Duchenne Muscular Dystrophy Mice by Elevating Cardiomyocyte Deoxy-Adenosine Triphosphate. JACC Basic Transl Sci. 2019 Oct 2;4(7):778-791. doi: 10.1016/j.jacbts.2019.06.006.

2. Powers JD, Yuan CC, McCabe KJ, Murray JD, Childers MC, Flint GV, Moussavi-Harami F, Mohran S, Castillo R, Zuzek C, Ma W, Daggett V, McCulloch $A D$, Irving TC, Regnier M. Cardiac myosin activation with 2-deoxy-ATP via increased electrostatic interactions with actin. Proc Natl Acad Sci U S A. 2019 Jun 4;116(23):11502-11507. doi: 10.1073/pnas.1905028116.

3. Nowakowski SG, Regnier M, Daggett V. Molecular mechanisms underlying deoxy-ADP.Pi activation of pre-powerstroke myosin. Protein Sci. 2017 Apr;26(4):749-762. doi: 10.1002/pro.3121.

4. Kadota S, Carey J, Reinecke H, Leggett J, Teichman S, Laflamme MA, Murry CE, Regnier M, Mahairas GG. Ribonucleotide reductase-mediated increase in dATP improves cardiac performance via myosin activation in a large animal model of heart failure. Eur J Heart Fail. 2015 Aug;17(8):772-81. doi: 10.1002/ejhf.270.

5. Kolwicz SC Jr, Odom GL, Nowakowski SG, Moussavi-Harami F, Chen X, Reinecke H, Hauschka SD, Murry CE, Mahairas GG, Regnier M. AAV6mediated Cardiac-specific Overexpression of Ribonucleotide Reductase Enhances Myocardial Contractility. Mol Ther. 2016 Feb;24(2):240-250. doi: 10.1038/mt.2015.176.

$$
* * * * *
$$

\#62. May 28, 2021

\section{A mitochondrial therapy for muscular dystrophies}

Marco Schiavone (1), Alessandra Zulian (1), Anna Stocco (1), Natalia Smolina (1), Valeria Petronilli (1), Justina Šileikytė (2), Michael Forte (2), Michael Cohen (3), Jordan Devereaux (4), Francesco Argenton (5), Luciano Merlini (6), Patrizia Sabatelli (7), Paolo Bernardi (1)*

(1) Department of Biomedical Sciences and CNR Neuroscience Institute, University of Padova, Padova, Italy; (2) Vollum Institute, Oregon Health and Science University, Portland, Oregon; (3) Department of Physiology \& Pharmacology, Oregon Health and Science University, Portland, Oregon; (4) Medicinal Chemistry Core, Oregon Health \& Science University, 
Portland, Oregon; (5) Department of Biology, University of Padova, Padova, Italy; (6) Department of Biomedical and Neuromotor Sciences, University of Bologna, Bologna, Italy; (7) CNR Institute of Molecular Genetics and Laboratory of Musculoskeletal Cell Biology, Istituto Ortopedico Rizzoli-IRCCS, Bologna, Italy

* Paolo Bernardi: paolo.bernardi@unipd.it

Duchenne Muscular Dystrophy (DMD) is a severe degenerative muscle disease with early onset caused by mutations in the dystrophin gene. Dystrophin is mainly expressed in skeletal and cardiac muscle fibers, where it is localized under the sarcolemma in association with proteins of the dystroglycan complex to form the dystrophin-associated protein complex. Lack of dystrophin increases the susceptibility to formation of micro-tears and fragmentation, which is followed by fiber death and regeneration but also by inflammation and repair. The latter process results in fibrosis, which eventually predominates as the regeneration potential decreases. Deregulation of $\mathrm{Ca}^{2+}$ homeostasis leading to increased levels of intracellular $\mathrm{Ca}^{2+}$ is generally accepted as an initiating event ${ }^{1}$ that can contribute to activation of calpains and degradation of muscle proteins with an effect that is synergistic with oxidative and nitrosative stress. Mitochondrial dysfunction appears early with decreased respiration and ATP production affecting both relaxation and repair of sarcolemmal injury and decreased ability to buffer $\mathrm{Ca}^{2+}$. The long-term effect appears to be a vicious cycle of mitochondrial $\mathrm{Ca}^{2+}$ overload and ATP depletion eventually leading to muscle fiber death. ${ }^{2}$ In a variety of muscle diseases, including DMD, $\mathrm{Ca}^{2+}$-dependent mitochondrial dysfunction is linked to the permeability transition pore (PTP), an inner membrane channel the opening of which requires matrix $\mathrm{Ca}^{2+}$ and is favored by oxidants, while it is antagonized by $\mathrm{Mg}^{2+}$, adenine nucleotides, reducing agents and acidic pH3. In vertebrates, mitochondrial cyclophilin (CyP) D is a key regulator that favors PTP opening. Pore desensitization by CyPD inhibition has been the basis for the use of cyclosporin (Cs) A in a variety of PTPdependent diseases. ${ }^{3}$ Encouraging results have been obtained in various disease models but efficacy of CsA may be more limited in the long term, possibly due to the untoward effects of calcineurin inhibition on fiber switch to the more resistant oxidative type. Nonimmunosuppressive derivatives of CsA like NIM811 and Debio 025 (alisporivir), which lack the ability to inhibit calcineurin but maintain the PTP-desensitizing properties, are quite effective in muscle disease models. ${ }^{2}$ A caveat is in order with this strategy as well, however, because at least sixteen CyP isoforms are known in mammals and all will be affected by this class of inhibitors. We have therefore developed novel PTP inhibitors that do not act through CyPD are a very promising treatment for experimental models of Collagen VI myopathy and DMD. ${ }^{4,5}$ I will provide an update on the pharmacological inhibition of the PTP in models of muscular dystrophy as well as in cells and muscle fibers derived from patients.

Keywords: mitochondria; permeability; calcium deregulation; muscular dystrophy; fiber death.

\section{References}

1. Wrogemann K, Pena SD. Mitochondrial calcium overload: A general mechanism for cell-necrosis in muscle diseases. Lancet 1976; 1:672-674.

2. Zulian A, Schiavone M, Giorgio V, Bernardi P. Forty years later: Mitochondria as therapeutic targets in muscle diseases. Pharmacol Res 2016; 113:563-573.

3. Bernardi $P$, Rasola A, Forte $M$, Lippe $G$. The mitochondrial permeability transition pore: Channel formation by F-ATP synthase, integration in signal transduction, and role in pathophysiology. Physiol Rev 2015; 95:1111-1155.

4. Stocco A, Smolina N, Sabatelli P, Šileikyte J, Artusi $E$, Mouly $V$, Cohen $M$, Forte $M$, Schiavone $M$, Bernardi P. Treatment with a triazole inhibitor of the mitochondrial permeability transition pore fully corrects the pathology of sapje zebrafish lacking dystrophin. Pharmacol Res 2021;105421.

5. Sileikyte $J$, Devereaux $J$, de Jong $J$, Schiavone $M$, Jones K, Nilsen A, Bernardi P, Forte M, Cohen M. Second generation inhibitors of the mitochondrial permeability transition pore with improved plasma stability. ChemMedChem 2019; 14:1771-1782.

\#63. May 28, 2021

Exercise and nutrition concomitant to Enzyme Replacement Therapy are efficacious in adult Pompe patients.

\section{Corrado Angelini}

Department of Neuroscience, University of Padova, Italy

* Corrado Angelini: corrado.angelini@unipd.it

Pompe Disease also known as glycogenosis type 2 is due to deficiency in lysosomal alpha-glucosidase, a lysosomal hydrolase and presents infantile and late onset variants (LOPD). The myopathy in LOPD can be reversed by ERT, but also by a high protein and aerobic exercise therapy. From 65 Late onset Pompe cases, we were able to obtain a self-reported evaluation from 58, most of them gave a positive efficacy evaluation of Enzyme Replacement Therapy (ERT) and they were classified by a self administered scale as Responders (R) or non Responders (NR).By a cooperative clinical group age, sex, BMI, GMW scale and 6MWT were monitored, however the only clinical parameters that were significantly associated with a Responder category were the use of regular diet, exercise or both. The present study, shows that in LOPD the condition can be reversed by ERT, but also benefits from concomitant diet and aerobic exercise therapy. 
Keywords: Glycogenosis type 2; exercise; nutrition.

1. Clinical Trial Slonim AE, Bulone L, Goldberg T, Minikes J, Slonim E, Galanko J, Martiniuk F. Modification of the natural history of adult-onset acid maltase deficiency by nutrition and exercise therapy. Muscle Nerve. 2007 Jan;35(1):70-7. doi: 10.1002/mus.20665.

2. Angelini C, Nascimbeni AC, Semplicini $C$. Therapeutic advances in the management of Pompe disease and other metabolic myopathies Ther Adv Neurol Dis. 2013 Sep;6(5):311-21. doi: 10.1177 /1756285613487570.

3. van der Ploeg AT, Kruijshaar ME, Toscano A, Laforêt P, Angelini C, Lachmann RH, Pascual Pascual SI, Roberts M, Rösler K, Stulnig T, van Doorn PA, Van den Bergh PYK, Vissing J, Schoser B; European Pompe Consortium. European consensus for starting and stopping enzyme replacement therapy in adult patients with Pompe disease: a 10year experience Review. Eur J Neurol. 2017 Jun;24(6):768-e31. doi: 10.1111/ene. 13285. Epub 2017 May 6.

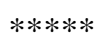

\#64. May 28, 2021

In vivo recovery of $\alpha$-sarcoglycan mutants by protein folding correctors

Martina Scano (1)*, Alberto Benetollo (1), Michela Bondì (1), Paola Caccin (1), Marcello Carotti (1), Francesco Dalla Barba (1), Michela Soardi (1), Roberta Sacchetto (2), Dorianna Sandonà (1)

(1) Department of Biomedical Sciences, University of Padova, Italy; (2) Department of Comparative Biomedicine and Food Science, University of Padova, Italy

* Martina Scano: martina.scano@phd.unipd.it

Sarcoglycanopathies are rare genetic diseases in which the disruption of the sarcoglycan complex results in sarcolemma fragility and progressive muscle degeneration. Most of the reported cases are due to missense mutations originating a folding-defective sarcoglycan (SG) eliminated by the cells' quality control system, although potentially functional. ${ }^{1}$ The molecular mechanism of these diseases has been recently elucidated allowing to envisage novel therapeutic possibilities. To recover the mutants and avoid complex disruption, we exploited the use of protein folding correctors belonging to the CFTR modulators family. The effective rescue of different SG-mutants has been proved for a few of such molecules by using cell models and, importantly, myogenic cells from sarcoglycanopathy patients. ${ }^{2,3}$ To validate the pharmacological strategy in vivo we have been forced to generate novel animal models of the disease expressing a folding-defective SG, overcoming the unsuitability of the available mouse models. ${ }^{4,5}$ To this intent, we transduced with AAV-SGCA hind-limbs of $\alpha$ SG null mouse pups resulting in transiently "humanized hind-limbs". Indeed, the adeno associated viral transduction resulted in hind-limbs expressing the endogenous $\beta-, \gamma-, \delta$-SG, and the human $\alpha$-SG (wild type or mutated) carried by the virus. By this way, we met the need of generating more than one model of the disease, and we had the possibility to control the amount of the mutated $\alpha$-SG expressed by the virus. No major effects on survival, body weight and general behavior have been observed in transduced animals. Histological and molecular characterization of the hind-limb muscles from 2 months old mice evidenced the recovery of the dystrophic phenotype when the wild-type sequence of the human $\alpha$-SG was transduced, while the presence of a mutated $\alpha$-SG resulted in pathologic features. Mice with "humanized hind-limbs" were then chronically treated with the most promising CFTR corrector, by intra peritoneal injection. The molecular and histological analyses of samples revealed an increase of the $\alpha$-SG content, a reduction of the signs of myopathy and the relocalization of the SG-complex at the sarcolemma in comparison to the vehicle treated animals. No sign of toxicity was observed during treatment. Even though a great variability is present, this is the first in vivo evidence of the efficacy of CFTR correctors in sarcoglycanopathy.

Keywords: sarcoglycanopathies; novel mouse models; CFTR correctors.

\section{References}

1. Carotti M, Fecchio C, Sandonà D. Emerging therapeutic strategies for sarcoglycanopathy. Expert Opinion on Orphan Drugs 5: 381-396, 2017.

2. Bianchini E, Fanin $M$, Mamchaoui $K$, Betto $R$, Sandonà $D$. Unveiling the degradative route of the V247M $\alpha$-sarcoglycan mutant responsible for LGMD-2D. Hum Mol Genet. 2014 Jul 15;23(14):3746-58. doi: 10.1093/hmg/ddu088. Epub 2014 Feb 23.

3. Carotti M, Marsolier J, Soardi M, Bianchini E, Gomiero C, Fecchio C, Henriques SF, Betto R, Sacchetto R, Richard I, Sandonà D. Repairing folding-defective $\alpha$-sarcoglycan mutants by CFTR correctors, a potential therapy for limb-girdle muscular dystrophy 2D. Hum Mol Genet. 2018 Mar 15;27(6):969-984. doi: 10.1093/hmg/ddy013.

4. Kobuke K, Piccolo F, Garringer KW, Moore SA, Sweezer E, Yang B, Campbell KP. A common diseaseassociated missense mutation in alpha-sarcoglycan fails to cause muscular dystrophy in mice. Hum Mol Genet. 2008 May 1;17(9):1201-13. doi: 10.1093/ hmg/ddn009. Epub 2008 Feb 5.

5. Henriques SF, Patissier C, Bourg N, Fecchio C, Sandona D, Marsolier J, Richard I. Different outcome of sarcoglycan missense mutation between human and mouse. PLoS One. 2018 Jan 
23;13(1):e0191274. doi: 10.1371/journal.pone. 0191274.

\#65. May 28, 2021

Proteomic identification of novel biomarkers of dystrophinopathy

Stephen Gargan $(1,2) *$, Paul Dowling $(1,2)$, Dieter Swandulla (3), Kay Ohlendieck $(1,2)$

(1) Department of Biology, Maynooth University, National University of Ireland, Maynooth W23F2H6, Co. Kildare, Ireland; (2) Kathleen Lonsdale Institute for Human Health Research, Maynooth University, Maynooth W23F2H6, Co. Kildare, Ireland; (3) Institute of Physiology II, University of Bonn, D53115 Bonn, Germany.

* Stephen Gargan: stephen.gargan@mu.ie

Duchenne muscular dystrophy is the most common neuromuscular disorder of early childhood and characterized by multi-systemic complications that are caused by a primary abnormality in the X-chromosomal DMD gene. In order to identify novel biomarker candidates of the diverse pathophysiological aspects of dystrophinopathy, our laboratories have initiated the systematic survey of body-wide alterations in muscular dystrophy. Mass spectrometry-based proteomics of the established mdx-4cv mouse model of Duchenne muscular dystrophy forms the basis of our analytical approach. Data sets from comparative proteomic investigations are routinely analyzed by systems bioinformatics to determine potential dystrophinopathyrelated changes at the level of protein families, biochemical pathways and protein-protein interaction patterns. Proteomic changes in components of special interest are verified by independent methodology, including comparative immunoblotting, immunofluorescence microscopy and enzyme assays. Our systematic analysis has focused on the proteomic analysis of various skeletal muscles (diaphragm, various predominantly fast-twitching versus slow-twitching limb muscles, extraocular muscle), cardiac muscle and smooth muscle, as well as the brain, liver, kidney, spleen and pancreas. In addition, the biofluid proteome of serum, saliva and urine were investigated for disease-specific changes in protein constituents. This bioanalytical strategy has identified a large number of muscle-related biomarker candidates involved in fiber contraction, the physiological regulation of the excitation-contractionrelaxation cycle, ion homeostasis, fiber metabolism, cytoskeletal organization, adaptations of the extracellular matrix and the cellular stress response. Importantly, many indirect changes were identified to occur in the liver, kidney and spleen that correlate well with bodywide alterations in energy metabolism, physiological regulation and the immune response. Novel biomarkers will now be tested for improving diagnostic and therapymonitoring approaches.

Keywords: biomarker; Duchenne muscular dystrophy; mass spectrometry; proteomics.

\section{References}

1. Dowling $P$, Murphy S, Zweyer M, Raucamp $M$, Swandulla D, Ohlendieck K. Emerging proteomic biomarkers of $X$-linked muscular dystrophy. Expert Rev Mol Diagn. 2019 Aug;19(8):739-755. doi: 10.1080/14737159.2019.1648214. Epub 2019 Aug 2.

2. Gargan S, Dowling P, Zweyer M, Swandulla D, Ohlendieck K. Identification of marker proteins of muscular dystrophy in the urine proteome from the $m d x-4 c v$ model of dystrophinopathy. Mol Omics. 2020 Jun 1;16(3):268-278. doi: 10.1039/c9mo00182d. Epub 2020 Mar 25..

3. Dowling P, Gargan S, Zweyer M, Henry M, Meleady $P$, Swandulla D, Ohlendieck K. Proteome-wide Changes in the $m d x-4 c v$ Spleen due to Pathophysiological Cross Talk with DystrophinDeficient Skeletal Muscle. iScience. 2020 Aug 26;23(9):101500. doi: 10.1016/j.isci.2020.101500. Epub ahead of print.

4. Murphy S, Zweyer $M$, Raucamp $M$, Henry $M$, Meleady P, Swandulla D, Ohlendieck K. Proteomic profiling of the mouse diaphragm and refined mass spectrometric analysis of the dystrophic phenotype. $J$ Muscle Res Cell Motil. 2019 Mar;40(1):9-28. doi: 10.1007/s10974-019-09507-z. Epub 2019 Mar 19.

5. Dowling $P$, Zweyer $M$, Raucamp $M$, Henry $M$, Meleady P, Swandulla D, Ohlendieck K. Proteomic and cell biological profiling of the renal phenotype of the mdx-4cv mouse model of Duchenne muscular dystrophy. Eur J Cell Biol. 2020 Jan;99(1):151059. doi: 10.1016/j.ejcb.2019.151059. Epub 2019 Nov 18.

\#66. May 28, 2021

The alarmin HMGB1 links oxidative stress and inflammation in muscular dystrophy

Giorgia Careccia (1,2),* Marielle Saclier (3), Mario Tirone (4), Elena Ruggieri (1,2), Elisa Principi (5), Lizzia Raffaghello (5), Silvia Torchio (3), Deborah Recchia (6), Monica Canepari (6), Andrea Gorzanelli (4), Michele Ferrara (1), Patrizia Castellani (7), Anna Rubartelli (7), Patrizia Rovere-Querini (8), Maura Casalgrandi (9), Alessandro Preti (9), Isabella Lorenzetti (10), Claudio Bruno (5), Roberto Bottinelli (6, 11), Silvia Brunelli (12), Stefano Carlo Previtali, (10), Marco Emilio Bianchi (2, 4), Graziella Messina (3), Emilie Vénéreau (1) **

(1) Division of Genetics and Cell Biology, Tissue Regeneration \& Homeostasis Unit, IRCCS San Raffaele Scientific Institute, Milan, Italy; (2) San Raffaele University, Milan, Italy; (3) Department of Biosciences, University of Milan, Italy; (4) Division of 
Genetics and Cell Biology, Chromatin Dynamics Unit, IRCCS San Raffaele Scientific Institute, Milan, Italy; (5) Center of Translational and Experimental Myology, IRCCS Istituto Giannina Gaslini, Genova, Italy; (6) Department of Molecular Medicine, University of Pavia, Pavia, Italy; (7) Unità di Biologia Cellulare, IRCCS Ospedale Policlinico San Martino, Genova, Italy. (8) Division of Immunology, Transplantation and Infectious Immunity, IRCCS Ospedale San Raffaele, Milan, Italy. (9) HMGBiotech srl, Milan, Italy; (10) Division of Neuroscience and Inspe, Neuromuscular Repair Unit, IRCCS San Raffaele Scientific Institute, Milan, Italy. (11) Interdepartmental Centre for the Study of Biology and Sports Medicine, University of Pavia; Fondazione Salvatore Maugeri (IRCCS), Scientific Institute of Pavia, Pavia, Italy; (12) School of Medicine and Surgery, University of Milano-Bicocca, Monza, Italy

* Giorgia Careccia: careccia.giorgia@hsr.it

** Emilie Vénéreau: venereau.emilie@hsr.it

Muscular Dystrophies (MDs) are a group of genetic diseases characterized by progressive muscle wasting associated to oxidative stress and persistent inflammation. It is essential to deepen our knowledge on the mechanism connecting these two processes because current treatments for MDs have still limited efficacy and/or are associated with side effects. Here, we identified the alarmin High Mobility Group Box 1 (HMGB1) as a functional link between oxidative stress and inflammation in MDs. We previously demonstrated that the oxidation of HMGB1 cysteines switches its extracellular activities from the orchestration of tissue regeneration to the exacerbation of inflammation. ${ }^{1,2} \mathrm{We}$ now found that the balance between HMGB1 redox isoforms dictates whether skeletal muscle is in an inflamed or regenerating state, and we propose the rebalancing of HMGB1 redox isoforms expression as a possible therapeutic approach to counteract the progression of the dystrophic phenotypeKeywords: inflammation; oxidative stress; muscular dystrophies; HMGB1.

\section{References}

1. Venereau E, Casalgrandi $M$, Schiraldi $M$, Antoine DJ, Cattaneo A, De Marchis F, Liu J, Antonelli A, Preti A, Raeli L, Shams SS, Yang H, Varani L, Andersson U, Tracey KJ, Bachi A, Uguccioni M, Bianchi ME. Mutually exclusive redox forms of HMGB1 promote cell recruitment or proinflammatory cytokine release. Mutually exclusive redox forms of HMGB1 promote cell recruitment or proinflammatory cytokine release. $J$ Exp Med. 2012 Aug 27;209(9):1519-28. doi: 10.1084/jem.20120189.

2. Tirone $M$, Tran NL, Ceriotti C, Gorzanelli A, Canepari M, Bottinelli R, Raucci A, Di Maggio S, Santiago C, Mellado M, Saclier M, François S,
Careccia G, He M, De Marchis F, Conti V, Ben Larbi S, Cuvellier S, Casalgrandi M, Preti A, Chazaud B, Al-Abed Y, Messina G, Sitia G, Brunelli S, Bianchi $M E$, Vénéreau E. High mobility group box 1 orchestrates tissue regeneration via CXCR4. J Exp Med. 2018 Jan 2;215(1):303-318. doi: 10.1084/jem.20160217.

$$
* * * * *
$$

\#67. May 28, 2021

Chanarin-Dorfman Syndrome: novel patients and review of the literature

Murat Durdu,* Daniela Tavian (2,3), Corrado Angelini (4), Sara Missaglia $(2,3)^{* *}$

(1) Neuromuscular Laboratory, Department of Neurosciences, University of Padova, Italy; (2) Laboratory of Cellular Biochemistry and Molecular Biology, CRIBENS, Università Cattolica del Sacro Cuore, Italy; (3) Psychology Department, Università Cattolica del Sacro Cuore, Italy; (4) Baskent University Faculty of Medicine, Department of Dermatology, Adana Hospital, Turkey.

* Sara Missaglia: sara.missaglia@unicatt.it

$\alpha / \beta$-hydrolase domain-containing protein 5 (ABHD5) is a lipid droplet-associated protein that promotes the hydrolysis of triacylglycerols (TAGs) by activating adipose triglyceride lipase (ATGL). ATGL is a lipase which catalyzes the initial and rate-limiting step in lipolysis by removing the first fatty acid from TAGs ${ }^{1}$. ABHD5 gene mutations lead to Chanarin-Dorfman syndrome (CDS), a rare condition in which TAGs accumulate in various tissues, including skin, liver and muscle ${ }^{2}$. To date, 147 CDS patients have been described. In these subjects, systemic involvement may manifest as ichthyosis, hepatosplenomegaly and/or liver steatosis, muscle weakness, hypotonia or muscle cramps, nystagmus, cataracts and/or strabismus, deafness, and mental retardation ${ }^{3-4}$. Here we describe two novel patients from two unrelated Turkey families. Both subjects are males presenting widespread ichthyosis, grade I hepatosteatosis and myopathy. Mutation analysis reveals a frameshift mutation of ABHD5 gene, in homozygous status, causing the production of a truncated protein (p.N209*) which loses the putative interacting domain required for ATGL activation. p.N209* is the most common ABHD5 mutation, in particular in Turkish patients $(26 / 39)^{5}$. Since it is difficult to interpreter ABHD5 genetic variants in CDS patients, the description of novel CDS cases might contribute to clarify genotypephenotype correlation.

Keywords: Lipid disorder; Chanarin-Dorfman Syndrome; ichthyosis; liver involvement; myopathy.

References 
1. Missaglia S, Coleman RA, Mordente A, Tavian D. Neutral Lipid Storage Diseases as Cellular Model to Study Lipid Droplet Function. Cells. 2019;8(2):187. doi:10.3390/cells8020187.

2. Lefevre $C$, Jobard F, Caux F, Bouadjar B, Karaduman A, Heilig R, Lakhdar H, Wollenberg A, Verret JL, Weissenbach J, Ozgüc $M$, Lathrop $M$, Prud'homme JF, Fischer J. Mutations in CGI-58, the gene encoding a new protein of the esterase/lipase/thioesterase subfamily, in ChanarinDorfman syndrome. Am J Hum Genet. 2001;69:1002-12. doi: 10.1086/324121.

3. Pennisi EM, Arca M, Bertini E, Bruno C, Cassandrini $D$, D'amico A, Garibaldi M, Gragnani F, Maggi L, Massa R, Missaglia S, Morandi L, Musumeci $O$, Pegoraro E, Rastelli E, Santorelli FM, Tasca E, Tavian D, Toscano A, Angelini C; Italian NLSD Group. Clinical/genetic features and natural history in a large cohort of Italian patients. Orphanet J Rare Dis. 2017;12:90. doi: 10.1186/s13023-017-0646-9.

4. Eskiocak AH, Missaglia S, Moro L, Durdu M, Tavian D. A novel mutation of ABHD5 gene in a Chanarin Dorfman patient with unusual dermatological findings. Lipids Health Dis. 2019;18(1):232. doi: 10.1186/s12944-019-1181-6.

5. Durdu M, Missaglia S, Moro L, Tavian D. Clinical and genetic characterization of a Chanarin Dorfman Syndrome patient born to diseased parents. BMC Med Genet. 2018;19(1):88. doi: 10.1186/s12881018-0610-0.

\#68. May 28, 2021

Crosstalk between MФs and resident cells in a dystrophic mouse model of MФ depletion

Alessio Torcinaro (1)*, Luca Madaro (2), Federica Francesca Contino (1), Marco De Bardi (3), Mattia Pelizzola (4), Giuseppe Diaferia (5), Pier Lorenzo Puri (6), Francesca De Santa (1)**

(1) Institute of Biochemistry and Cell Biology (IBBC), National Research Council of Italy (CNR), Monterotondo (Rome), Italy; (2) DAHFMO- Unit of Histology and Medical Embryology, Sapienza University of Rome, Rome, Italy; (3) IRCCS Fondazione Santa Lucia (FSL), Rome, Italy; (4) Center for Genomic Science of IIT@SEMM, Fondazione Istituto Italiano di Tecnologia (IIT), Milan, Italy; (5) IRCCS European Institute of Oncology (IEO), Milan, Italy; (6) Development, Aging and Regeneration Program, Sanford Burnham Prebys Medical Discovery Institute, La Jolla, CA, USA

\section{* Alessio Torcinaro: alessio.torcinaro@libero.it \\ ** Francesca De Santa: francesca.desanta@cnr.it}

Duchenne Muscular Dystrophy (DMD) is a lethal Xlinked disease that determines high oxidative stress levels, persistent inflammation, myogenic defects and increasing fibrosis and fat deposition within muscles. In this scenario, dysregulation of cellular crosstalk among cell populations involved in muscle regeneration results crucial. ${ }^{1,2}$ Specifically, stem cell niche of satellite cells (SCs), muscle stem cells, is finetuned by immune cells, mainly represented by macrophages (MФs) and interstitial cells, like fibroadipogenic progenitors (FAPs). ${ }^{3,4}$ The experimental design took advantage of a dystrophic mouse model of transient and inducible M $\Phi$ depletion recently generated in our laboratory ( $\mathrm{mdx}^{\text {ITGAM-DTR }}$ ). The research plan also included a transcriptomic approach based on genome-wide expression profiles of whole muscle and muscle resident cells. ${ }^{5}$ We analyzed the effect of modulation of inflammatory environment on muscle regeneration, focusing on the impact on function of SCs and FAPs. Our data suggest that the modulation of MФs in dystrophic muscles exerts a deep impact on regeneration leading to an exacerbated phenotype (5). Moreover, we revealed a direct involvement of MФs in preserving SC and FAP number and identity counteracting, in turn, muscle degeneration. We also identified a novel cell population, referred to as $\alpha 7 \mathrm{Sca} 1$, in $\mathrm{M} \Phi$-depleted muscles of $\mathrm{mdx}^{\mathrm{ITGAM}-\mathrm{DTR}}$ mice. Considering that, the characterization of the role of macrophages will provide crucial information to design and fine-tune therapeutic approaches targeting inflammatory component in dystrophic muscles.

Keywords: Crosstalk between MФs; resident cells; dystrophic mouse model of MФ depletion.

\section{References}

1. Evans NP, Misyak SA, Robertson JL, BassaganyaRiera J, Grange RW. Dysregulated intracellular signaling and inflammatory gene expression during initial disease onset in Duchenne muscular $d$ ystrophy. Am J Phys Med Rehabil. 2009;88(6):502522. doi:10.1097/PHM.0b013e3181a5a24f

2. Mann CJ, Perdiguero E, Kharraz Y, et al. Aberrant repair and fibrosis development in skeletal muscle. Skelet Muscle. 2011;1(1):21. Published 2011 May 4. doi:10.1186/2044-5040-1-21

3. Bentzinger CF, Wang YX, Dumont NA, Rudnicki MA. Cellular dynamics in the muscle satellite cell niche. EMBO Rep. 2013;14(12):1062-1072. doi:10.1038/embor.2013.182

4. De Santa F, Vitiello L, Torcinaro A, Ferraro E. The Role of Metabolic Remodeling in Macrophage Polarization and Its Effect on Skeletal Muscle Regeneration. Antioxid Redox Signal. 2019;30(12):1553-1598. doi:10.1089/ ars.2017.7420

5. Madaro L, Torcinaro A, De Bardi $M$, et al. Macrophages fine tune satellite cell fate in dystrophic skeletal muscle of mdx mice. PLoS Genet. 2019;15(10):e1008408. Published 2019 Oct 18. doi:10.1371/journal.pgen.1008408 
\#69. May 29, 2021

\section{Evidence- based balneotherapy applications in} musculoskeletal disorders

Stefano Masiero (1), Maria Chiara Maccarone $(1,2)$, Lucrezia Tognolo (1)

(1) Rehabilitation Unit, Department of Neuroscience, University of Padova, Padua, Italy; (2) Physical Medicine and Rehabilitation School, University of Padova, Padua, Italy

* Stefano Masiero: stef.masiero@unipd.it

Balneotherapy is a traditional therapeutic modality that exploits natural methods for the treatment and the prevention of several conditions. During the centuries, it has been extensively applied and its effects have been studied in detail. Balneotherapy is frequently used in patients with musculoskeletal disorders (MSDs). In these patients balneotherapy interventions such as mud baths, mineral-rich water immersions, in-water exercises, etc. have been widely used for years. Our purpose is to explore recent evidence concerning balneotherapy interventions in MSDs in order to define if they can represent appropriate strategies to treat these patients. We screen PubMed, MEDLINE and Google Scholar databases using as keywords: balneotherapy, balneology, spa therapy, Health resort medicine, peloidotherapy, and musculoskeletal disorders. The results demonstrate the beneficial effects of balneotherapy on musculoskeletal function, inflammatory parameters, pain perception, and Quality of Life in patients with MSDs. Balneotherapy, especially if combined with rehabilitation interventions and strategies for health education, can significantly improve MSDs symptoms, pro-inflammatory substrate, and psychological concerns of patients with chronic MSDs. Combining the specific chemical effects of mineral-rich waters and the well-known physical properties, in addition to the effects on mental wellbeing, peculiar to the surrounding natural environment, balneotherapy interventions can be used to improve the health status of patients affected by MSDs, representing an effective alternative to in-hospital rehabilitative interventions.

Keywords: Balneotherapy; balneology; spa therapy; Health resort medicine; peloidotherapy; musculoskeletal disorders.

\section{References}

1. Masiero S, Vittadini F, Ferroni C, Bosco A, Serra R, Frigo AC, Frizziero A. The role of thermal balneotherapy in the treatment of obese patient with knee osteoarthritis. Int J Biometeorol. 2018 Feb;62(2):243-252. doi: 10.1007/s00484-017-14457. Epub 2017 Sep 22.

2. Masiero S. Thermal rehabilitation and osteoarticular diseases of the elderly. Aging Clin Exp Res. 2008;20(3):189-194. doi:10.1007/BF03324772.
3. Gálvez I, Torres-Piles S, Ortega-Rincón E. Balneotherapy, Immune System, and Stress Response: A Hormetic Strategy?. Int J Mol Sci. 2018;19(6):1687. Published 2018 Jun 6. doi:10.3390/ijms19061687.

4. Maccarone MC, Magro G, Solimene U, Masiero S. Spa therapy can improve quality of life in chronic musculoskeletal disorder subjects: a narrative review. Bulletin of rehabilitation medicine. 2020; 96 (2): 3-6. doi:10.38025/ 2078-1962-2020-96-2-3-6.

5. Antonelli M., Donelli D., Fioravanti A. Effects of balneotherapy and spa therapy on quality of life of patients with knee osteoarthritis: a systematic review and meta-analysis. Rheumatol Int. 2018: 38(10): 1807 - 1824. doi: 10.1007/s00296-018-4081-6.

$$
* * * * *
$$

\#70. May 29, 2021

Anti-inflammatory properties of the Euganean thermal muds: the role of the patented microbiota

\section{Nicoletta La Rocca}

Department of Biology, University of Padova, Italy

* Nicoletta La Rocca: nicoletta.larocca@unipd.it

The Euganean Thermal District (Italy) represents the oldest and largest thermal district in Europe and its therapeutic mud is considered a unique product whose beneficial effects are documented since Ancient Romans times. Mud properties are ascribed to heat and electrolytes of the thermal water as well as on antiinflammatory molecules produced by its biotic component. The therapeutic muds are in fact obtained from natural clays applying a traditional maturation process that leads to the growth of a green microbial biofilm with Cyanobacteria as fundamental components. The investigation of the healing effects of compounds produced by these Cyanobacteria is an important goal for scientific validation of Euganean mud therapies and in general for the discovering of new health beneficial biomolecules. So far, it has been demonstrated that Phormidium sp. ETS-05, the most abundant cyanobacterium of the mature muds, produce lipids such as monogalactosyldiacylglycerol (MGDG), that showed anti-inflammatory activity in vitro and in vivo (mouse). ${ }^{1,2}$ For this reason, Phormidium sp. ETS-05 is considered the target species of the mud maturation process and the effectiveness of its principles led to obtain a European Patent of the mud therapeutic efficacy. ${ }^{3}$ More recently, we also investigated the therapeutic potential of exopolysaccharides (EPS) produced by Phormidium sp. ETS05, finding that they have anti-inflammatory and pro-resolution activities in vivo (zebrafish), confirming the high value of Euganean mud treatments for chronic inflammatory diseases recovery. ${ }^{4}$ Moreover, we performed an in-depth study on the microbiota colonizing the Euganean thermal muds. ${ }^{5}$ We analyzed 53 mature muds from 29 sites (Spas) using a polyphasic 
approach, finding a microbiota stable and peculiar of the area with a Cyanobacteria population composition dominated by Phormidium sp. ETS-05. We also obtained the complete genome sequence of this target species using a mixed sequencing approach based on Illumina and Nanopore sequencing. This will be the base for future studies to understand how mud maturation operating parameters could influence the production of the active therapeutic molecules (lipids and polysaccharides).

Keywords: Euganean Thermal District; Cyanobacteria; anti-inflammatory bioactive molecules; monogalactosyldiacylglycerols; exopolysaccharides.

\section{References}

1. Bruno A, Rossi C, Marcolongo G, Di Lena A, Venzo $A$, Berrie CP, Corda D. Selective in vivo antiinflammatory action of the galactolipid monogalactosyldiacylglycerol. Eur J Pharmacol. 2005 Nov 7;524(1-3):159-68. 10.1016/j.ejphar.2005.09.023. Epub 2005 Oct 25.

2. Ulivi V, Lenti M, Gentili C, Marcolongo G, Cancedda $R$, Descalzi Cancedda F. Anti-inflammatory activity of monogalactosyldiacylglycerol in human articular cartilage in vitro: activation of an anti-inflammatory cyclooxygenase-2 (COX-2) pathway. Arthritis Res Ther. 2011 Jun 17;13(3):R92. doi: 10.1186/ar3367.

3. Lalli, A., Andreoli, C., Ceshi Berrini, C., De Appolonia, F., Marcolongo, G. (2013) Antiinflammatory active principles in Euganean thermal mud. Patent number EP1571203 (B1).

4. Zampieri RM, Adessi A, Caldara F, Codato A, Furlan $M$, Rampazzo C, De Philippis R, La Rocca N, Dalla Valle L. Anti-Inflammatory Activity of Exopolysaccharides from Phormidium sp. ETS05, the Most Abundant Cyanobacterium of the Therapeutic Euganean Thermal Muds, Using the Zebrafish Model. Biomolecules. 2020 Apr 10;10(4):582. doi: 10.3390/biom10040582.

5. Gris B, Treu L, Zampieri RM, Caldara F, Romualdi C, Campanaro S, La Rocca N. Microbiota of the Therapeutic Euganean Thermal Muds with a Focus on the Main Cyanobacteria Species. Microorganisms. 2020 Oct 15;8(10):1590. doi: 10.3390/microorganisms 8101590.

\#71. May 29, 2021

Histological study of deltoid muscle after mud therapy

Olga Surdu (1), Cezarina Mihaela Mehedinti (2), Virgiliu Surdu (1,3), Garofita Olivia Mateescu (4)

(1) Balneal And Rehabilitation Sanatorium of Techirghiol; (2) Dunarea de Jos University, Faculty of Medicine, Galati; (3) Ovidius University, Faculty of Medicine, Constanta; (4) University of Medicine and Pharmacy Craiova, Romania

* Olga Surdu: olga.surdu@yahoo.com
Question: does mud application produce histological modification in muscle tissue? Thirty five in-patients underwent twelve days of peloidotherapy in Techirghiol during the year 2005. The study was approved by the bioethics commission of the university and inclusion and exclusion criteria and informed consent were applied. The cropping was made from the left deltoid area and fragments were histologic classical worked, examined with a Nikon E-600 microscope and acquired with a Sony video camera. The images were processed using the LUCIA@ $C$ G soft of image analysis. The general aspect of muscle tissue shows an increased number of open capillaries, congestion of blood vessels and presence of neoangiogenesis vessels. Patients who received peloidotherapy presented an increased number of blood vessels on the microscopic field, statistically significant $\mathrm{P}(\mathrm{t})<0,01$. These result on muscle study after peloidotherapy were never previously communicated or published. In conclusion, neoangiogenesis vessels are the evidence of histological modification induced by peloidotherapy. The increased number of open capillaries at the end of balneal treatment illustrates the fact that heat exchange stimulates the thermoregulation and vascular function and thus produces, beside functional hyperemia, new vessels.

Keywords: histological study; deltoid muscle; mud therapy.

\section{References}

1. Mogoanţă L., et al, „, Ghid de tehnici de histologie, citologie şi imunohistichimie”, Ed. Medicală Universitară, Craiova 2015, ISBN 973-8354-71-4, 56-57;

2. Mehedinţi T., „Histologie”, ed. Ovidius University Press, Constanţa, 1998, ISBN 973-9367-11-9, 86 - 110;

3. Mureşan P., "Manual de metode matematice în analiza stării de sănătate, ed. Medicală, Bucureşti, 1989, 151-167.

$$
* * * * *
$$

\#72. May 29, 2021

\section{Balneology \& Balneotherapy in Russia,}

Anatoly Dmitrievich Fesyun, Andrey Rachin,

Ministry of Healthcare of Russian Federation, Moscow, Russian Federation

* Anatoly Dmitrievich Fesyun: nmicrk@nmicrk.ru

\#73. May 29, 2021

American Balneology - updates in the field of North American Balneology and the evolution from recreation to medicine

\section{Marcus Coplin}

Naturopathic Heaithcare, San Francisco, CA, USA

* Marcus Coplin: drmarcuscoplin@gmail.com 
North American Balneology has largely been focused on wellness and recreation for the past 75 years. Academic research and medical applications are largely absent from the contemporary field. The Balneology Association of North America (BANA) is working with thermal health centers, natural spring managers, and potential patients throughout North America to educate and grow the understanding of the benefits of Balneology. Focusing on convalescent care in a post-COVID world, and the recategorization of thermal mineral waters as a therapeutic agent, BANA aims to see North America rejoin the international community standards for the research and application of Balneology.

Keywords: Historical; Convalescence; Essential; Therapeutic Physical Agent.

\section{References}

1. Historical Sources. Journal of Balneology and Medical Clippings Volume 3, Issue 61889

2. William RH. A Handbook of Climatic Treatment Including Balneology 1906. THE JOURNAL BALNEOLOGY AND DIETARY Volume 4 December 1890

3. Xu L, Wu L, Liu T, Xing W, Cao X, Zhang S, Su Z. Effect of a 21-day balneotherapy program on blood cell counts, ponogen levels, and blood biochemical indexes in servicemen in sub-health condition. J Phys Ther Sci. 2017 Sep;29(9):1573-1577. doi: 10.1589/jpts.29.1573. Epub 2017 Sep 15. PMID: 28931990; PMCID: PMC5599823.

4. Wątor, K., Dobrzyński, D., Sugimori, K. et al. Redox potential research in the field of balneochemistry: case study on equilibrium approach to bioactive elements in therapeutic waters. Int J Biometeorol 64, 815-826 (2020). https://doi.org/10.1007/s00484020-01871-4)

5. Melgar-Sánchez LM, García-Ruiz I, Pardo-Marqués V, Agulló-Ortuño MT, Martínez-Galán I. Influence of mineral waters on in vitro proliferation, antioxidant response and cytokine production in a human lung fibroblasts cell line. Int J Biometeorol. 2019 Sep;63(9):1171-1180. doi: 10.1007/s00484-01901730-0. Epub 2019 Jun 21. PMID: 31227887.

$$
* * * * *
$$

\#74. May 29, 2021

Prevention and rehabilitation exemplified by the combination of TCM, conventional Western medicine and the therapeutic effect of alkaline Glauber's salt

\section{Rainer Blaser}

Stiftung Gesundheitsförderung Bad Zurzach + Baden, Bad Zurzach, Switzerland

\section{* Rainer Blaser: r.blaser@stiftungbadzurzach.ch}

The Gemeinnützige Stiftung Zurzacher Kuranlagen was set up at the instigation of Dr. Walter Edelmann and his wife in 1957. The establishment of this charitable foundation was prompted by the discovery during drilling operations of a hot spring at a depth of more than $400 \mathrm{~m}$. Specifically, this turned out to be an alkaline Glauber's salt thermal spring whose waters, with a temperature of 40 degrees, have excellent healing properties. The foundation's aim and purpose was to cover future developments in the field of medicine. Ultimately, this led to today's foundation group, the multi-site expansion, the current name and the objective of encouraging health promotion in the areas of prevention + rehabilitation and spa + wellness. The Rheumatism and Rehabilitation Clinic (today's RehaClinic) in Bad Zurzach is the foundation's greatest achievement. Alongside conventional Western medicine, its focus now increasingly lies on the inclusion of complementary treatment methods. Traditional Chinese Medicine (TCM) Ming Dao is also a member of the foundation group. Together with the RehaClinic, it adopts an approach that marries conventional Western medicine with traditional Chinese medicine. The RehaClinic in Bad Zurzach was the first of its kind to incorporate TCM into its therapies by making it an established part of the inpatient rehabilitation process in 1995. Following 10 years of successful collaboration between conventional medicine and TCM in treating inpatients, this tried-and-tested concept was also rolled out to a number of outpatient centers. Using a combination of TCM and conventional medicine allows patients to receive therapeutic support for a wide variety of conditions. One of the hallmarks of TCM is its ability to treat more than 90 different diseases. These include strokes, dizziness, sleep problems, facial palsy, colds, rheumatic conditions, pain syndromes, sciatica, shoulder problems, pain in general, menstrual and fertility problems, depression, hay fever, tinnitus and sore throats. The combination of meridian acupuncture, acupressure, cupping, warm wraps and herbal remedies, along with mobilizing methods such as tai chi, qigong, kung fu or the five elements nutrition theory, successfully produces a long-term therapeutic effect at both the physical and mental level. The healing waters of the thermal springs also play a key role in the area of prevention and rehabilitation. The alkaline Glauber's salt has both a preventive and pain-relieving effect. The Bad Zurzach Glauber's salt spring is used in the treatment of musculoskeletal disorders (rheumatoid arthritis), neurological disorders (stroke, paralysis, Parkinson's), vascular disease brought on by cancer and tumor diseases as well as chronic pain. Various exercise baths with different water temperatures and a spacious outdoor pool underscore the importance of the Glauber's salt spring in the recovery process. It alleviates rheumatic complaints, especially wear and tear of the spine, the joints and soft tissues. In addition, bathing has a positive effect on movement and circulatory disorders, as well as on residual paralysis of the central and peripheral nervous system. The water is also suitable for drinking to treat 
gallbladder, liver and stomach ailments. That is why its effect, and especially its preventive effect, when used in thermal bath treatments is also the subject of ongoing research within TCM prevention. The foundation continually invests in education and research in order to safeguard and integrate the benefits and effects of complementary medicine.

Keywords: prevention and rehabilitation; complementary medicine; TCM; conventional Western medicine; alkaline Glauber's salt.

\section{References}

1. Altendorf Hans-Dietrich, Hidber Alfred, Sennhauser Albert, et al. Geschichte des Fleckens Zurzach. Verlag Historische Vereinigung des Bezirks Zurzach 2004.

2. Bär Andrea, Angst Felix, Li Yiming. Traditionelle Chinesische Medizin bei rheumatischen Krankheiten. Haug Verlag 2004.

3. Li Yiming, Qi Kai, Aeschlimann André G., et al. Traditionelle Medizinische Kultur. Lebensgewohnheiten zur Förderung von Gesundheit. Verlag Amazon 2015.

$$
* * * * *
$$

\#75. May 29, 2021

\section{Masticatory muscles instrumental evaluation in the dental practice}

Elena P Ivanova, (1), Riccardo Rosati (2).

(1) National Medical Research Center of Rehabilitation and Balneology of Health Ministry of Russia, Moscow, Russia; (2) University of Milan, Italy

* Elena P Ivanova: IvanovaEP@nmicrk.ru

Severe tooth wear, crowding, loss of masticatory units and oncologic surgical resections are the most common situations that require restoration of patient dental occlusion. ${ }^{1-3}$ Any dental work that changes the occlusal surfaces and/or the tooth position could potentially modify oral proprioception. Several study demonstrate how the interdental perception threshold in healthy subjects is less than 2 hundredths of a millimeter. Dental treatments involving occlusal surfaces could change oral proprioception sometimes forcing neuromuscular patterns to develop functional adaptations. Dental afferents play a role in the masticatory muscles recruitment than occlusal modifications could change masticatory muscles global contraction intensity but also their functional relationship. Indeed muscle imbalances may originate from several factors including a higher number of contacts, interference in the working or balancing side and loss of posterior vertical support. ${ }^{4}$ It has been also demonstrated how proprioceptive dental alterations may require also neck muscles functional adaptations. ${ }^{5}$ Great number of subjects fortunately has a wide functional adaptability indeed the relationship between abnormal muscle recruitment and symptoms such as pain or signs of dysfunction like movement limitation does not appear linear. The absence of clinical symptoms (mainly pain) following an occlusal intervention does not means a procedure free from imperfections and/or anomalies. The muscles and nervous tissue adaptability to new oral conditions (without causing symptoms such as pain) could "mask" changes in other structures, for example, teeth, bones and joints. ${ }^{6}$ Surface electromyography (sEMG) is a low-cost, non-invasive method usable in research and in dental clinical practice for the quantitative and qualitative analysis of head and neck muscles. ${ }^{7}$ Standardized sEMG indexes allow to evaluate occlusal-induced proprioceptive mediated muscular recruitment in a reliable way than merging biomechanical concepts with sEMG standardized indexes, occlusal devices and prosthesis adapting procedures could be clinically performed in order to reduce the muscular adaption to the new occlusal conditions or to re-establish physiological muscular coordination. ${ }^{8}$ Standardized surface electromyography (ssEMG) protocols for static (clenching) and dynamic (chewing and swallowing) evaluation in the general dentistry practice and craniomandibular-dysfunction will be discussed.

Keywords: masticatory muscles; instrumental evaluation; dental practice.

\section{References}

1. Botelho AL, Gentil FH, Sforza C, da Silva MA. Standardization of the electromyographic signal through the maximum isometric voluntary contraction. Cranio. 2011 Jan;29(1):23-31. doi: 10.1179/crn.2011.005.

2. Benninger B, Lee BI. Clinical importance of morphology and nomenclature of distal attachment of temporalis tendon. J Oral Maxillofac Surg. 2012 Mar;70(3):557-61. doi: 10.1016/j.joms.2011.02.047. Epub 2011 May 6.

3. Chang Y, Cantelmi D, Wisco JJ, Fattah A, Hannam AG, Agur AM. Evidence for the functional compartmentalization of the temporalis muscle: a 3dimensional study of innervation. J Oral Maxillofac Surg. 2013 Jul;71(7):1170-7. doi: 10.1016/j.joms. 2013.04.005.

4. Trovato F, Orlando B, Bosco M. Occlusal features and masticatory muscles activity. A review of electromyographic studies. Stomatologija. 2009;11(1):26-31.

5. Ferrario VF, Sforza C, Dellavia C, Tartaglia GM. Evidence of an influence of asymmetrical occlusal interferences on the activity of the sternocleidomastoid muscle. J Oral Rehabil. 2003 Jan;30(1):34-40. doi: 10.1046/j.1365-2842.2003. 00986.x.

6. Le Bell Y, Jämsä T, Korri S, Niemi PM, Alanen P. Effect of artificial occlusal interferences depends on previous experience of temporomandibular 
disorders. Acta Odontol Scand. 2002 Aug;60(4):21922. doi: 10.1080/000163502760147981.

7. Dong $Y$, Wang XM, Wang MQ, Widmalm SE. Asymmetric muscle function in patients with developmental mandibular asymmetry. $J$ Oral Rehabil. 2008 Jan;35(1):27-36. doi: 10.1111/j.13652842.2007.01787.x.

8. Rosati R, Semelewa E, Satygo E, Ivanova EP, Rosati $F$, Silin A, Ferrario VF. Standardized electromyographic indexes allow a reliable measurement of masticatory muscles function. Stoma Edu J. 2016;3(2):235-242.

$* * * * *$

\#76. May 29, 2021

Modulation of respiratory function in a patient with angina pectoris using transcutaneous auricular nerve stimulation

Janez Rozman $(1,2)$ *, Polona Pečlin (3)

(1) Center for Implantable Technology and Sensors, ITIS d. o. o. Ljubljana, Slovenia; (2) Institute of Pathophysiology, Faculty of Medicine, University of Ljubljana, Slovenia; (3) University Medical Centre Ljubljana, Division of Gynecology and Obstetrics, Ljubljana, Slovenia

* Janez Rozman: janez.rozman@guest.arnes.si

Transcutaneous auricular nerve stimulation (tANS) is a combined diagnostic and treatment technique, based on normalizing the body's dysfunction through the stimulation of the auricular branch of the vagus nerve at specific sites on the external ear. tANS that potentially influences afferent nerve activity can act directly on the nerve fibers and thus alters the airway physiology. This study aims to measure the effects of selective tANS at predefined sites for both cymba concae (CC) on respiratory function, assuming that it could be altered with the tANS. The protocols were approved by the National Medical Ethics Committee, Ministry of Health, Republic of Slovenia (Unique Identifier No. 0120297/2018/6). Written, informed consent was obtained from the 62-year-old male subject with angina pectoris. The devices for the tANS are the plugs that contain four platinum stimulating cathodes, a common anode (AXELGAARD MANUFACTURING CO., LTD., Fallbrook, CA 92028, USA), and a microprocessorcontrolled stimulator with two independent outputs (Shenzhen L-Domas Technology Ltd., Shenzhen, Guangdong, China). The air-flow measuring system was developed to assess the variations in the airflow, the changes in the rhythm and the character of the respiration produced by the selective tANS. This system comprised a full-face mask and a ventilator flow sensor (HAMILTON MEDICAL, Hamilton Medical AG, Bonaduz, Switzerland) to measure the pressure difference elicited by the bi-directional nasal airflow using a modified transducer for invasive blood-pressure monitoring (Smiths Medical Deutschland $\mathrm{GmbH}$, Germany). The temporal parameters of the rectangular current biphasic stimulating pulses were the following: Frequency $45.5 \mathrm{~Hz}$, Stimulating phase width $200 \mu \mathrm{s}$, Anodic phase width $200 \mu$ s, Interphase delay $180 \mu$ s, Pulse train duration $2.0 \mathrm{~s}$ and Time gap between successive pulse trains $1.0 \mathrm{~s}$. The intensity was pre-set until the minimum discomfort at the site below the deployed cathode was detected. The tANS trials were conducted on a daily basis: one site on the left or right CC a day. Each 20-minute trial started with the 5-minute placebo, proceeded with the 10-minute tANS and ended with the 5-minute placebo. The assessed signals fed to a data-acquisition system (DEWESOFT d.o.o., Republic of Slovenia) were gathered at $200 \mathrm{kHz}$ and stored on a portable computer (Lenovo, Beijing, China). To evaluate the effect of tANS each of three segments within a particular recording, were analysed manually. In an offline analysis, an average of the entities calculated within the particular time period are the following: Breath length calculated from readings during 10 breaths, Peak-to-peak air flow calculated from readings during 10 breaths and finally, Maximum slope of air flow calculated from readings during 10 breaths. The study demonstrated that the selective tANS had either a positive or negative effect on the respiratory function in all the trials. The highest positive and the lowest negative effects were observed in the trial accomplished at the site indicated as left black, while the lowest positive and highest negative effects were observed in the trial accomplished at the site indicated as left yellow. In other trials, these effects were found to be between the above two results.

Keywords: Transcutaneous auricular-nerve stimulation; external ear; respiratory function.

References

1. Butt MF, Albusoda A, Farmer AD, Aziz Q. The anatomical basis for transcutaneous auricular vagus nerve stimulation. J Anat. 2020 Apr;236(4):588-611. doi: 10.1111/joa.13122. Epub 2019 Nov 19.

2. Corey J, Pallanch J. Evaluation of Nasal Breathing Function with Objective Airway Testing, Cummings Otolaryngology-Head and Neck Surgery, 2010; 640656. doi: 10.1016/B978-0-323-05283-2.00043-4.

3. Eccles R. Nasal airflow in health and disease. Acta Otolaryngol. 2000 Aug;120(5):580-95. doi: 10.1080 1000164800750000388.

4. Henry TR. Therapeutic mechanisms of vagus nerve stimulation. Neurology. 2002 Sep 24;59(6 Suppl 4):S3-14. doi: 10.1212/wnl.59.6_suppl_4.s3.

5. Kuhn A, Keller T, Lawrence M, Morari M. A model for transcutaneous current stimulation: simulations and experiments. Med Biol Eng Comput. 2009 Mar;47(3):279-89. doi: 10.1007/s11517-008-0422-z. Epub 2008 Nov 13.

$* * * * *$ 
\#77. May 29, 2021

Adjunctive Physiotherapy as a complement to Balneology - A case report on the management and effective treatment of Diabetic Neuropathic Foot Ulcer using adjunctive physiotherapy

\section{Marcus Coplin}

Naturophatic Heaithcare, San Francisco, CA, USA

* Marcus Coplin: drmarcuscoplin@gmail.com

Balneology is a primarily physical therapeutic agent. Physiotherapy must be considered as an adjunctive medical service for long term health and rehabilitation to be administered at Thermal Health Centers. A review of adjunctive physiotherapy methods will be presented along with a case report of the successful treatment of a Diabetic Neuropathic Foot Ulcer scheduled for surgical amputation, by therapeutically applying nonbalneological physical agents.

Keywords: Iontophoresis; Pulsed-Shortwave Diathermy; Galvanic Current; Physical Agents.

\section{References}

1. Guo L, Kubat NJ, Isenberg RA. Pulsed radio frequency energy (PRFE) use in human medical applications. Electromagn Biol Med. 2011 Mar;30(1):21-45. doi: 10.3109/15368378.2011. 566775.

2. Hunckler J, de Mel A. A current affair: electrotherapy in wound healing. $J$ Multidiscip Healthc. 2017;10:179-194. Published 2017 Apr 20. doi:10.2147/JMDH.S127207.

3. Barnes R, Shahin Y, Gohil R, Chetter I. Electrical stimulation vs. standard care for chronic ulcer healing: a systematic review and meta-analysis of randomised controlled trials. Eur J Clin Invest. 2014 Apr;44(4):429-40. doi: 10.1111/eci.12244.

4. Lundeberg TC, Eriksson SV, Malm M. Electrical nerve stimulation improves healing of diabetic ulcers. Ann Plast Surg. 1992 Oct;29(4):328-31. doi: 10.1097/00000637-199210000-00009.

5. Tan GZ, Orndorff PE, Shirwaiker RA. The Ion Delivery Manner Influences the Antimicrobial Efficacy of Silver Oligodynamic Iontophoresis. J Med Biol Eng. 2019;39: 622-631. doi: 10.1007/ s40846018-0447-1.

\#78. May 29, 2021

Invited Lecture: Exercise and the regulation of mitophagy in muscle

\section{David Hood}

Muscle Health Research Centre, York University, Toronto, ON, Canada

* David Hood: dhood@yorku.ca
Mitochondria are dynamic organelles that are constantly being synthesized and recycled. This "turnover" promotes the maintenance of an optimally functioning pool of mitochondria. In response to acute exercise, signaling cascades are initiated to upregulate PGC- $1 \alpha$, as well as other transcriptional regulators, which increase the transcription of nuclear genes encoding mitochondrial proteins. These gene products are translated and incorporated into preexisting organelles, which can then fuse with the mitochondrial reticulum. When mitochondrial dysfunction arises, evident from the loss of membrane potential and increased ROS emission, these organelle segments undergo fission from the functional network and are subject to mitophagy and recycling, aided by the lysosome. The most common pathway for mitochondrial degradation involves the activation of Pink1 and Parkin. Mitochondria tagged by this pathway are engulfed in autophagosomes and then transported to lysosomes, where the two will fuse and the contents of the autophagosome will be broken down by lysosomal enzymes. Exercise is a stimulus for mitochondrial targeting, lysosomal biogenesis, and subsequent degradation of autophagosomes, and can serve to repair mitochondrial dysfunction via this pathway. This Seminar will focus on the regulation of mitophagy with exercise, involving both PGC- $1 \alpha$ as well as the Pink1-Parkin pathway.

Keywords: muscle; exercise; aging; PGC-1 $\alpha$; Pink1Parkin; mitochondrial adaptations.

\section{References}

1. Hood DA, Memme JM, Oliveira AN, Triolo M. Maintenance of Skeletal Muscle Mitochondria in Health, Exercise, and Aging. Annu Rev Physiol. 2019 Feb 10;81:19-41. doi: 10.1146/annurev-physiol020518-114310. Epub 2018 Sep 14.

$$
* * * * *
$$

\#79. May 29, 2021

Parvalbumin knock out as a model of the mitochondrial control of cytosolic calcium in skeletal muscle fibers

Carlo Reggiani, Anna Raffaello, Denis Vecellio Reane, Marta Canato

Department of Biomedical Sciences, University, of Padova, Italy

* Carlo Reggiani: carlo.reggiani@unipd.it

The mitochondrial calcium uniporter (MCU) complex with its regulatory subunits provides mitochondria with a highly specialized channel which allows a fast uptake of calcium from the cytosol as soon as cytosolic calcium concentration rises. It is widely accepted that, in skeletal muscle fibers, calcium taken up by mitochondria plays a significant regulatory role in a variety of processes from metabolism regulation to muscle fiber trophism and to 
apoptosis (see for review, Raffaello et al. 2016). ${ }^{1}$ In contrast it is still debated whether MCU can contribute to shape the cytosolic calcium transients in skeletal muscle during excitation-contraction (E-C) coupling. The close apposition of mitochondria to the calcium release units (CRU) can be definitely instrumental to this function. There are two factors playing against this possibility: 1 ) the timing, i.e. whether the mitochondrial calcium uptake is sufficiently fast to modify a calcium release-removal cycle which lasts less than $100 \mathrm{~ms}$ and 2) the amount of calcium which can be stored in the mitochondrial matrix, as the mitochondria could store only a small fraction of the hundreds of micromoles of calcium released by the sarcoplasmic reticulum. Taken together these two factors have supported the view that mitochondria are not relevant for a quantitative regulation of intracellular calcium dynamics (Cannell and Allen 1984, ${ }^{2}$ Baylor and Hollingworth $2012^{3}$ ). There are, however, a few studies showing that under specific conditions mitochondria can contribute significantly. Gillis (1997) ${ }^{4}$ showed that local application of Ruthenium Red $(50 \mu \mathrm{M})$ was able to slow down the relaxation process in intact fibers of rabbit masseter where mitochondria cover $16 \%$ of fiber volume and in rat soleus muscle. More recently, Weiss and coworkers $(2010)^{5}$ showed that FCCP application in rat FDB induced not only delayed relaxation but also increased the level of resting cytosolic calcium concentration. Consistently, Yi and coworkers (2011) ${ }^{6}$ showed a prolongation of the cytosolic calcium transient induced by voltage clamp in FDB fibers of a mouse model of amyotrophic lateral sclerosis (ALS). Muscle fibers of this murine strain show localized mitochondrial defects involving loss of inner membrane potential linked to an elevated and poorly controlled intracellular calcium release activity. In the present study we aimed to assess the contribution of mitochondria to control cytosolic calcium transient in FDB muscle fibers of mice carrying a null mutation of parvalbumin (PV) (Schwaller et al 1999) ${ }^{7}$. PV is the major cytosolic calcium buffer, able to bind an amount of calcium comparable to that bound by C-troponin, although with a slower kinetics. Thus, PV removal is expected to create a significant unbalance in cytosolic calcium homeostasis. Our experimental results showed that, in PV null fibers compared to WT fibers, no changes in amplitude but only changes in kinetics of the cytosolic calcium transients induced by electrical stimulation were detectable. To assess the potential contribution of mitochondria, we modified the mitochondrial capacity to take up calcium by silencing the MCU, along the line of previous studies (Mammucari et al. 2015). ${ }^{8}$ In single muscle fiber of FDB MCUsilenced PV null, but not in MCU-silenced WT fibers, we observed a higher peak of cytosolic calcium following a calcium release induced either by electrical stimulation or by caffeine $(10 \mathrm{mM})$ administration. Our results add further evidence to the view that mitochondria are able to contribute to cytosolic calcium regulation in skeletal muscle fibers and, under suitable conditions, their contribution becomes a determinant of amplitude and kinetics of calcium transients.

Keywords: Parvalbumin knock out mice; mitochondrial control of cytosolic calcium; skeletal muscle fibers.

\section{References}

1. Raffaello A, Mammucari C, Gherardi G, Rizzuto R. Calcium at the Center of Cell Signaling: Interplay between Endoplasmic Reticulum, Mitochondria, and Lysosomes. Trends Biochem Sci. 2016 Dec;41(12): 1035-1049. doi: 10.1016/j.tibs.2016.09.001.

2. Cannell MB, Allen DG. Model of calcium movements during activation in the sarcomere of frog skeletal muscle. Biophys J. 1984 May;45(5):913-25. doi: 10.1016/S0006-3495(84)84238-1.

3. Baylor SM, Hollingworth S. Intracellular calcium movements during excitation- contraction coupling in mammalian slow-twitch and fast-twitch muscle fibers. $J$ Gen Physiol. 2012 Apr;139(4):261-72. doi: 10.1085/jgp.201210773.

4. Gillis JM. Inhibition of mitochondrial calcium uptake slows down relaxation in mitochondria-rich skeletal muscles. J Muscle Res Cell Motil. 1997 Aug;18(4):47383. doi: 10.1023/a:1018603032590.

5. Weiss N, Andrianjafiniony T, Dupré-Aucouturier S, Pouvreau S, Desplanches D, Jacquemond V. Altered myoplasmic $\mathrm{Ca}^{2+}$ handling in rat fast-twitch skeletal muscle fibres during disuse atrophy. Pflugers Arch. 2010 Mar;459(4):631-44. doi: 10.1007/s 00424-009-0764-x.

6. Yi J, Ma C, Li Y, Weisleder N, Ríos E, Ma J, Zhou J. Mitochondrial calcium uptake regulates rapid calcium transients in skeletal muscle during excitationcontraction (E-C) coupling. J Biol Chem. 2011 Sep 16;286(37):32436-43. doi: 10.1074/jbc. M110.217711.

7. Schwaller B, Dick J, Dhoot G, Carroll S, Vrbova G, Nicotera $P$, Pette $D$, Wyss A, Bluethmann $H$, Hunziker $W$, et al. Prolonged contraction-relaxation cycle of fast-twitch muscles in parvalbumin knockout mice. Am. J. Physiol. Cell Physiol. 1999 Feb; 276(2):C395-403. doi: 10.1152/ajpcell.1999.276.2. C395.

8. Mammucari C, Gherardi G, Zamparo I, Raffaello A, Boncompagni S, Chemello F, Cagnin S, Braga A, Zanin $S$, Pallafacchina G, Zentilin L, Sandri M, De Stefani D, Protasi F, Lanfranchi G, Rizzuto R. The mitochondrial calcium uniporter controls skeletal muscle trophism in vivo. Cell Rep. 2015 Mar 3;10(8):1269-79. doi: 10.1016/j.celrep.2015.01.056.

$$
* * * * *
$$

\#80. May 29, 2021

\section{Why EM is still important in muscle research}

\section{Simona Boncompagni}

CAST, Center for Advanced Studies and Technology; University G D'Annunzio of Chieti-Pescara, Italy

* Simona Boncompagni: simona.boncompagni@unich.it After its commercial availability around the fifties and a large use and application, for a period of time electron microscopy (E.M.) went out of fashion and often it has 
been considered simply too descriptive to be worthy of publication. However, standard electron E.M. often provides the structural basis at the nanoscale level for unraveling functions and defining mechanisms of the cells. Because of his stereotyped striated architecture skeletal muscle provides a convenient model to show the utility of structure-function correlation. Indeed just to quote an old instance: the mechanisms of muscle contraction from the triggering of contraction to the sliding filaments theory (A. F. Huxley), ${ }^{1}$ excitationcontraction coupling, ${ }^{2,3}$ and calcium homeostasis could not have been elucidated without the structural foundation revealed by E.M. muscle. More recently, our laboratory provided evidence for mitochondria and calcium release units (CRUs) structural association, ${ }^{4}$ and described for the first time presence of new structural organelles contributing to $\mathrm{Ca}^{2+}$ influx during the Store Operated Calcium Entry (SOCE) mechanism. ${ }^{5}$ Using examples from published data and my extensive archive of micrographs, I illustrate how well performed E.M. analysis and electron micrographs are extensive sources of information in physiological or pathological conditions still in our modern time.

Keywords: Electron Microscopy (E.M.); skeletal muscle; ultrastructure.

\section{References}

1. Huxley AF. The activation of striated muscle and its mechanical response. roc $R$ Soc Lond B Biol Sci. 1971 Jun 15;178(1050):1-27. doi: 10.1098/rspb.1971.0049.

2. Porter KR, Palade GE. Studies on the endoplasmic Reticulum III. Its form and distribution in striated muscle cells. J Biophys Biochem Cytol. 1957 Mar 25;3(2):269-300. doi: 10.1083/jcb.3.2.269.

3. Franzini-Armstrong C. The membrane system of muscle cells. In Engel AG, Franzini-Armstrong C. Eds. Myology III Edition. 2004. McGrawHill NY, pp 232-256.

4. Boncompagni S, Rossi A E, Micaroni M, Beznoussenko GV, Polishchuk RS, Dirksen DT, Protasi F. Mitochondria are linked to calcium stores in striated muscle by developmentally regulated tethering structures. Mol Biol Cell. 2009 Feb;20(3):1058-67. doi: 10.1091/mbc.e08-070783. Epub 2008 Nov 26.

5. Boncompagni S, Michelucci A, Pietrangelo L, Dirksen RT, Protasi. F. Exercise-dependent formation of new junctions that promote STIM1-Orai1 assembly in skeletal muscle. Sci Rep. 2017 Oct 27;7(1):14286. doi: 10.1038/s41598-01714134-0.

\#81. May 29, 2021

Proper architecture of organelles deputed to $\mathrm{Ca}^{2+}$ handling and aerobic ATP production underlies efficient muscle function

\section{Feliciano Protasi}

CAST, Center for Advanced Studies and Technology; University G D'Annunzio of Chieti-Pescara, Italy

* Feliciano Protasi: feliciano.protasi@unich.it
Proper skeletal muscle function is controlled by intracellular $\mathrm{Ca}^{2+}$ concentration and by efficient production of energy (ATP), which in turn depend on: a) release and re-uptake of $\mathrm{Ca}^{2+}$ from intracellular stores during excitation-contraction (EC) coupling, which controls muscle contraction and relaxation; b) uptake of $\mathrm{Ca}^{2+}$ into the mitochondrial matrix, which stimulates aerobic ATP production; and finally c) entry of $\mathrm{Ca}^{2+}$ from the extracellular space via store-operated $\mathrm{Ca}^{2+}$ entry (SOCE), a mechanism important to limit muscle fatigue. Abnormalities in $\mathrm{Ca}^{2+}$ handling underlies many physiopathological conditions, including dysfunction in ageing. In the last 15 years in different, but complementary, projects we have collected the following results: a) denervation of muscle fibers - an event that contributes to some degree to sarcopenia - causes disarray of EC coupling membranes (calcium release units, CRUs) and of the mitochondrial network; ${ }^{1}$ b) sedentary ageing causes partial disarray of CRUs and calcium entry units (CEUs, dynamic structures involved in SOCE), and misplacement of mitochondria; ${ }^{2}$ c) functional electrical stimulation (FES) and exercise promotes rescue/maintenance of the proper architecture of CRUs, CEUs, and mitochondria in denervation and ageing. ${ }^{3,4}$ These data indicate that a) integrity and proper disposition of intracellular organelles deputed to $\mathrm{Ca}^{2+}$ handling and aerobic generation of ATP is challenged by inactivity or reduced activity; and b) disarranged architecture of the intracellular membrane systems may underline muscle dysfunction in ageing and sarcopenia.

\section{References}

1. Kern H, Boncompagni $S$, Rossini K, Mayr W, Fanò G, Zanin ME, Podhorska-Okolow M, Protasi F, Carraro U. Long-term denervation in humans causes degeneration of both contractile and excitation-contraction coupling apparatus, which is reversible by functional electrical stimulation (FES): a role for myofiber regeneration? J Neuropathol Exp Neurol. 2004 Sep;63(9):919-31. doi: 10.1093/jnen/63.9.919.

2. Boncompagni S, Kern H, Rossini K, Hofer C, Mayr W, Carraro U, Protasi F. Structural differentiation of skeletal muscle fibers in the absence of innervation in humans. Proc Natl Acad Sci U S A. 2007 Dec 4;104(49):19339-44. doi: 10.1073/pnas.0709061104. Epub 2007 Nov 27..

3. Pietrangelo L, D'Incecco A, Ainbinder A, Michelucci A, Kern H, Dirksen RT, Boncompagni S, Protasi F. Agedependent uncoupling of mitochondria from $\mathrm{Ca}^{2+}$ release units in skeletal muscle. Oncotarget. 2015 Nov 3;6(34):35358-71. doi: 10.18632/onco target.6139.

4. Pietrangelo L, Michelucci A, Ambrogini P, Sartini S, Guarnier FA, Fusella A, Zamparo I, Mammucari C, Protasi F, Boncompagni S. Muscle activity prevents the uncoupling of mitochondria from $\mathrm{Ca}^{2+}$ Release Units induced by ageing and disuse. Arch Biochem Biophys. 2019 Mar 15;663:22-33. doi: 10.1016/j.abb.2018.12. 017. Epub 2018 Dec 20.

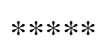


\#82. May 29, 2021

\section{Association of mitochondria and triads is maintained by muscle activity}

Laura Pietrangelo,* Simona Boncompagni, Feliciano Protasi,

CAST, Center for Advanced Studies and Technology; University G D'Annunzio of Chieti-Pescara, Italy

* Laura Pietrangelo: laura.pietrangelo@unich.it

$\mathrm{Ca}^{2+}$ release units (CRUs, also named triads), the sites of excitation-contraction coupling (EC) ${ }^{1}$ and mitochondria, the organelles deputed to produce ATP during cellular respiration, ${ }^{2}$ in fast-twitch fibers from adult mice are structurally linked by small strands, or tethers. ${ }^{3}$ The uptake of $\mathrm{Ca}^{2+}$ into the mitochondrial matrix is influenced by triad-mitochondria proximity and proposed to stimulate the respiratory chain., ${ }^{2,4}$ Aging causes separation of a fraction of mitochondria from CRUs and a cross-talk impairment between the two organelles. ${ }^{5}$ However, whether this uncoupling is the result of ageing per-se or the consequence of reduced muscle activity remains still unclear. In a paper published in $2019,{ }^{6}$ we tested if muscle activity maintains the correct association of mitochondria and triads, in a) extensor digitorum longus (EDL) muscles from 2 years old mice, either sedentary or trained for 1 year in wheel cages; and b) EDL muscles from denervated adult mice and rats. We analyzed muscle samples using a combination of structural, biochemical, and functional experimental procedures. The results collected in structural studies indicate that: a) ageing and denervation result in partial uncoupling between CRUs and mitochondria; b) exercise and re-innervation either maintains (in old mice) or restores (in transiently denervated rats and mice) the association between the two organelles. Functional studies support the hypothesis that CRU-mitochondria cross-talk is important for mitochondrial $\mathrm{Ca}^{2+}$ uptake. Taken together, our results suggest that muscle activity is necessary to maintain/improve the proper association between sites of $\mathrm{Ca}^{2+}$ release and mitochondria, which is important for correct signaling between the two organelles and, likely, for proper aerobic ATP production.

Keywords: excitation-contraction (EC) coupling; ageing; denervation; exercise.

\section{References}

1. Franzini-Armstrong C, Jorgensen AO. Structure and development of E-C coupling units in skeletal muscle. Аnnu Rev Physiol. 1994;56:509-34. doi: 10.1146/ annurev.ph.56.030194.002453.

2. Brookes PS, Yoon Y, Robotham JL, Anders MW, Sheu SS. Calcium, ATP, and ROS: a mitochondrial lovehate triangle. Am J Physiol Cell Physiol. 2004 Oct;287(4):C817-33. doi: 10.1152/ajpcell.00139.2004.
3. Boncompagni $S$, Rossi AE, Micaroni $M$, Beznoussenko GV, Polishchuk RS, Dirksen RT, Protasi F. Mitochondria are linked to calcium stores in striated muscle by developmentally regulated tethering structures. Mol Biol Cell. 2009 Feb;20(3):1058-67. doi: 10.1091/mbc.e08-07-0783. Epub 2008 Nov 26.

4. Rossi AE, Boncompagni S, Wei L, Protasi F, Dirksen RT. Differential impact of mitochondrial positioning on mitochondrial $\mathrm{Ca}^{2+}$ uptake and $\mathrm{Ca}^{2+}$ spark suppression in skeletal muscle. Am J Physiol Cell Physiol. 2011 Nov;301(5):C1128-39. doi: 10.1152/ajpcell.00194.2011. Epub 2011 Aug 17.

5. Pietrangelo L, D'Incecco A, Ainbinder A, Michelucci A, Kern H, Dirksen RT, Boncompagni S, Protasi F. Age-dependent uncoupling of mitochondria from $\mathrm{Ca}^{2+}$ release units in skeletal muscle. Oncotarget. 2015 Nov 3;6(34):35358-71. doi: 10.18632/onco target. 6139 .

\#83. May 29, 2021

\section{Mitochondrial metabolism in adulthood and ageing}

Gaia Gherardi, Rosario Rizzuto, Cristina Mammucari

Department of Biomedical Sciences, University of Padova, Italy

* Gaia Gherardi: gaia.gherardi88@gmail.com

The second messenger $\mathrm{Ca}^{2+}$ regulates a broad repertoire of cellular processes. Upon physiological stimuli, skeletal muscle mitochondria rapidly and efficiently accumulate $\mathrm{Ca}^{2+}$ into their matrix via an electrogenic pathway that relies on the driving force of a steep electrochemical gradient. A large $\left[\mathrm{Ca}^{2+}\right] \mathrm{mt}$ peak occurs dynamically in parallel to agonist-induced $\left[\mathrm{Ca}^{2+}\right] \mathrm{cyt}$ increases, thanks to the activity of the Mitochondrial Calcium Uniporter (MCU), the highly selective channel responsible for mitochondrial $\mathrm{Ca}^{2+}$ accumulation. ${ }^{1} \mathrm{MCU}$ positively regulates myofiber size in physiological conditions, and counteracts pathological loss of muscle mass. ${ }^{2}$ We have previously demonstrated that skeletal muscle-specific MCU deletion (MCU-/-) inhibits myofiber mitochondrial $\mathrm{Ca}^{2+}$ uptake, impairs muscle force and exercise performance. Mitochondrial Calcium uptake is required for effective glucose oxidation, as demonstrated by the fact that in muscle-specific MCU-/myofibers oxidative metabolism is impaired and glycolysis rate is increased. The decreased pyruvate dehydrogenase activity is the main trigger of this metabolic rewiring. Although defective, mitochondrial activity is partially sustained by increased fatty acid (FA) oxidation. $^{3}$ Here, we have investigated the role of mitochondrial $\mathrm{Ca}^{2+}$ uptake during skeletal muscle aging. We show that mitochondrial $\mathrm{Ca}^{2+}$ accumulation is decreased in 24 months old mice and this condition is accompanied by a decreased pyruvate dehydrogenase activity. We demonstrate a rewiring of skeletal muscle 
metabolism where mitochondrial activity is sustained by FA oxidation rather than glucose. Further studies are needed to evaluate whether the restoration of glucose as the main fuel for oxidative metabolism will be sufficient to counteract sarcopenia.

Keywords: mitochondrial $\mathrm{Ca}^{2+}$ uptake; ageing; skeletal muscle; aerobic metabolism.

\section{References}

1. Rizzuto $R$, De Stefani D, Raffaello A, Mammucari C. Mitochondria as sensors and regulators of calcium signalling. Nat Rev Mol Cell Biol. 2012 Sep;13(9):566-78. doi: 10.1038/nrm3412. Epub 2012 Aug 1.

2. Mammucari C, Gherardi G, Zamparo I, Raffaello A, Boncompagni S, Chemello F, Cagnin S, Braga A, Zanin S, Pallafacchina $G$, Zentilin L, Sandri $M$, De Stefani $D$, Protasi F, Lanfranchi G, Rizzuto $R$. The mitochondrial calcium uniporter controls skeletal muscle trophism in vivo. Cell Rep. 2015 Mar 3;10(8):1269-79. doi: 10.1016/j.celrep.2015.01.056. Epub 2015 Feb 26.

3. Gherardi G, Nogara L, Ciciliot S, Fadini GP, Blaauw B, Braghetta $P$, Bonaldo $P$, De Stefani D, Rizzuto $R$, Mammucari C. Loss of mitochondrial calcium uniporter rewires skeletal muscle metabolism and substrate preference. Cell Death Differ. 2019 Jan;26(2):362-381. doi: 10.1038/s41418-018-0191-7. Epub 2018 Sep 19.

\#84. May 29, 2021

Sarcoplasmic reticulum and mitochondria structural relationship is altered in early stages of cancerinduced cachexia

\section{Flávia A. Guarnier}

Department of General Pathology, Laboratory of Muscle Pathophysiology and Adaptation, Londrina State University, Londrina, Brazil.

\section{* Flávia A. Guarnier: faguarnier@uel.br}

Cancer cachexia is a complex multifactorial syndrome characterized by involuntary and pathological weight loss, mainly due to skeletal muscle wasting, and frequently associated with a profound fatigue. These effects are often blamed for decreasing patient's quality of life and survival. Statistics point to cachexia as a direct cause of death for $20-25 \%$ of all cancer patients. ${ }^{1}$ Regarding the pathophysiology of the syndrome, most of the data available in the literature is obtained through preclinical studies, which are carried out with very different experimental cancer models, most frequently at advanced cachexia stages. The most studied and best described models show decreasing in cross-sectional area of muscle fibers, and this atrophy is more likely to affect type II fibers. In addition, skeletal muscle ultrastructure appears to be impaired. Several studies have suggested an association between mitochondria dysfunction and skeletal muscle atrophy through disturbances in its dynamics, quality and function. These changes would precede muscle loss, while mitophagy would be present at later stages. ${ }^{2}$ The oxidative stress, that is related to myofibrils proteolysis and atrophy at different cachexia stages, ${ }^{3}$ is pointed out as mitochondrial modifications responsibility. ${ }^{4}$ In the last years, our group has dedicated attention to investigate oxidative and ultrastructural modifications in mitochondria and sarcoplasmic reticulum (SR), and correlate with muscle loss in glycolytic and oxidative muscles in a precocious stage of breast carcinosarcoma-induced cachexia. ${ }^{5}$ Recently collected data, show that, in a very early stage of an experimental model of cachexia: 1) glycolytic muscles are already more prone to mass waste, but independently of protein oxidation; 2) glycolytic fibers are already sensitive to mitochondria, and SR structural alterations; 3) these parameters are straightly correlated to membranes oxidative modifications; and 4) oxidative muscles are not preserved from oxidative and structural damage. We believe these new data bring to light an useful tool to fight this devastating syndrome.

Keywords: Cancer cachexia; oxidative modifications; skeletal muscle ultrastructure.

\section{References}

1. Warren $S$. The immediate causes of death in cancer. Am J Med Sci. 1932;184:610-615.

2. Brown JL, Rosa-Caldwell ME, Lee DE, Blackwell TA, Brown LA, Perry RA, Haynie WS, Hardee JP, Carson JA, Wiggs MP, Washington TA, Greene NP. Mitochondrial degeneration precedes the development of muscle atrophy in progression of cancer cachexia in tumour-bearing mice. J Cachexia Sarcopenia Muscle. 2017 Dec;8(6):926-938. doi: 10.1002/jcsm.12232. Epub 2017 Aug 28.

3. Guarnier FA, Cecchini AL, Suzukawa AA, Maragno $A L$, Simão AN, Gomes MD, Cecchini R. Time course of skeletal muscle loss and oxidative stress in rats with Walker 256 solid tumor. Muscle Nerve. 2010 Dec;42(6):950-8. doi: 10.1002/mus.21798.

4. Dolly A, Dumas JF, Servais S. Cancer cachexia and skeletal muscle atrophy in clinical studies: what do we really know? J Cachexia Sarcopenia Muscle. 2020 Dec;11(6):1413-1428. doi: 10.1002/jcsm. 12633. Epub 2020 Oct 14.

5. Blegniski FP, Vieira NA, Yamaguchi EY, Signori L, Bordini HP, Pecorai C, Protasi F, Gomes MD, Boncompagni S, Guarnier FA. Sarcoplasmic reticulum volume loss participation on fiber-specific skeletal muscle waste in precachexia induced by Walker-256 Carcinosarcoma. Annals of the III International Symposium of Experimental Pathology. 2019. ISBN: 978-85-302-0047-3.

$$
* * * * *
$$

\#85. May 29, 2021

See you to 2022 Padua Days on Muscle \& Mobility Medicine (PDM3), March 27-30, Thermae of Euganean Hills and Padova, Italy

Ugo Carraro $(1,2,3) *$ 
(1) Department of Biomedical Sciences of the University of Padova, Italy; (2) CIR-Myo - Myology Centre, University of Padova, Italy; (3) A\&C MioniCarraro Foundation for Translational Myology, Padova, Italy

\section{* Ugo Carraro: ugo.carraro@unipd.it}

Keywords: 2022 Padua Days on Muscle \& Mobility Medicine (PDM3); March 27-30, 2022; Thermae of Euganean Hills \& Padova, Italy.

See you to the 2022 Padua Days on Muscle \& Mobility Medicine (PDM3), March 27-30, Thermae of Euganean Hills \& Padova, Italy. I hope that massive vaccination will allow for in presence Meetings. If not we will continue with semi virtual or virtual Meetings. ${ }^{1,2}$ Traditional sessions will be: Sarcopenia \& Aging; Neuromuscular Disorders in Sarcopenia \& Cachexia; New Applications of TENS \& FES; Therapies for genetic diseases; Muscle \& Mobility Medicine Imaging; The Center of Active Aging; Rehabilitation of Mobility Disorders. New topics are welcome and I hope they will emerge during the incoming 2021 PDM3 May 26-29. Notice, please, that the deadline for submissions of Abstracts and Registrations will be September 30, 2021.

Keywords: Padua Days, Muscle, Mobility Medicine.

References

1 Carraro U. 2017 Spring Padua Muscle Days, roots and byproducts. Eur J Transl Myol. 2017 Jun 27;27(2):6810. doi: 10.4081/ejtm.2017.6810.24.

2. Carraro U. 30 Years of Translational Mobility Medicine: 2020 Padua Muscle Days go virtual from Euganean Hills, November 19th to 21st. Eur J Transl Myol. 2020 Nov 17;30(4):9437. doi: 10.4081/ejtm. 2020.9437.

\section{INDEX OF AUTHORS}

$\begin{array}{ll}\text { Adamo S } & 21 \\ \text { Adams ME } & 44 \\ \text { Agbulut } O & 21 \\ \text { Agresti A } & 29 \\ \text { Albracht K } & 10 \\ \text { Alves } J & 21 \\ \text { Amor } F & 43 \\ \text { Ancel S } & 41 \\ \text { Angelini } C & 28,49 \\ \text { Apuzzo LN } & 39 \\ \text { Arato I } & 31 \\ \text { Argenton F } & 45 \\ \text { Baccam A } & 21 \\ \text { Banerji CRS } & 38 \\ \text { Barton ER } & 40 \\ \text { Benetollo A } & 47 \\ \text { Benoni A } & 21 \\ \text { Bernardi } P & 45\end{array}$

$\begin{array}{ll}\text { Bianchi M } & 28 \\ \text { Bianchi ME } & 48 \\ \text { Bittmann FN } & 16,17,18 \\ \text { Biz C } & 4,6 \\ \text { Blaie S } & 43 \\ \text { Blaser R } & 53 \\ \text { Boncompagni S } & 57,59 \\ \text { Bondi D } & 24 \\ \text { Bondì M } & 47 \\ \text { Bottinelli R } & 48 \\ \text { Braun T } & 35 \\ \text { Brayson D } & 44 \\ \text { Brunelli S } & 48 \\ \text { Bruno C } & 48 \\ \text { Bugda Gwilt C } & 34 \\ \text { Burke SK } & 23 \\ \text { Butler-Browne G } & 42 \\ \text { Caccin P } & 47\end{array}$

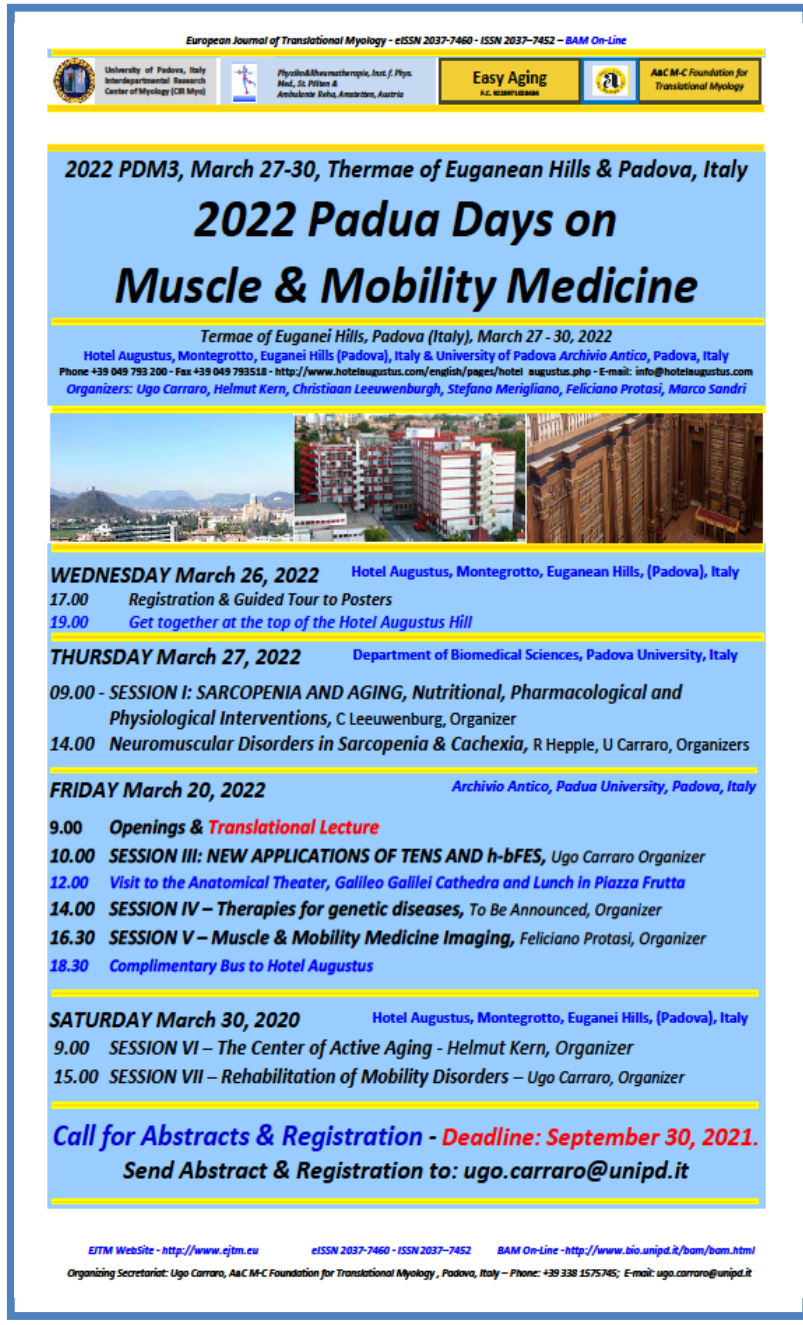

$* * * * *$ 


\begin{tabular}{|c|c|}
\hline Colombo F & 29 \\
\hline Contino FF & 50 \\
\hline Coplin M & 52,56 \\
\hline Costa $R$ & 28 \\
\hline Crooks S & 8 \\
\hline Dalla Barba F & 47 \\
\hline Day K & 36 \\
\hline De Bardi $M$ & 50 \\
\hline De Caro $R$ & 4,6 \\
\hline De Cesare $N$ & 10 \\
\hline De Santa F & 50 \\
\hline De Vito G & 14 \\
\hline $\operatorname{Dech} S$ & $16,17,18$ \\
\hline Del Vecchio A & 14 \\
\hline Devereaux $J$ & 45 \\
\hline Diaferia $G$ & 50 \\
\hline Dowling $P$ & 48 \\
\hline Duan D & 44 \\
\hline Durdu $M$ & 49 \\
\hline Edmunds KJ & 6 \\
\hline Engquist E & 38 \\
\hline Fan $C$ & 4,6 \\
\hline Fan $C-M$ & 36 \\
\hline Farina $D$ & 13 \\
\hline Fede C & 4,6 \\
\hline Feige $J$ & 41 \\
\hline Ferrara $M$ & 48 \\
\hline Fesyun $A D$ & 52 \\
\hline Fitzgerald L & 23 \\
\hline Fletcher JR & 8 \\
\hline Fontelonga $T$ & 34 \\
\hline Forte $M$ & 45 \\
\hline Franchi $M$ & 9 \\
\hline Freyler K & 10 \\
\hline Froehner SC & 44 \\
\hline Fulle S & 24 \\
\hline Gambacorta $M$ & 6 \\
\hline Ganassi M & 38 \\
\hline Garcia JM & 21 \\
\hline Gargan S & 48 \\
\hline Gargano C & 21 \\
\hline Gargiulo P & 6 \\
\hline Gerassimov $N$ & 36 \\
\hline Gherardi $G$ & 59 \\
\hline Gibbs D & 34 \\
\hline Girolami B & 28 \\
\hline Goldhamer DJ & 39 \\
\hline Gollhofer A & 10 \\
\hline Gorzanelli A & 48 \\
\hline Gregorevic $P$ & 26 \\
\hline Guarnier FA & 16,60 \\
\hline Guidolin D & 4,6 \\
\hline Guillaume C & 43 \\
\hline Gussoni E & 34 \\
\hline Hall A & 34 \\
\hline Hammers D & 42 \\
\hline Harrop A & 39 \\
\hline Hassani M & 21 \\
\hline
\end{tabular}

\begin{tabular}{|c|c|}
\hline Наи K-L & 44 \\
\hline Heher P & 38 \\
\hline Helm $M$ & 10 \\
\hline Hepple RT & 23 \\
\hline Herzog W & 8 \\
\hline Hong A-V & 43 \\
\hline Hood D & 56 \\
\hline Hoppeler $H$ & 20,27 \\
\hline Ibáñez J & 13 \\
\hline Israeli $D$ & 43 \\
\hline Ivanova EP & 54 \\
\hline Jacob D & 6 \\
\hline Jiang $E$ & 34 \\
\hline Johnson EK & 44 \\
\hline Judge A & 19 \\
\hline Judge S & 19 \\
\hline Kardon $G$ & 39 \\
\hline Kim $Y-J$ & 35 \\
\hline Kjaer $M$ & 3 \\
\hline Kok HJ & 40 \\
\hline La Rocca N & 51 \\
\hline Lattanzi G & 28 \\
\hline Lawlor M & 34 \\
\hline Lee $M$ & 42 \\
\hline Leeuwenburgh Ch & 18 \\
\hline Lepper Ch & 35 \\
\hline Li Z & 21 \\
\hline Lichtwark GA & 9 \\
\hline Lieber $R L$ & 7,11 \\
\hline Lorenzetti I & 48 \\
\hline Luca $G$ & 31 \\
\hline Maccarone MC & 51 \\
\hline Macchi V & 4,6 \\
\hline Macintosh BR & 8 \\
\hline Madaro L & 50 \\
\hline Mammucari C & 59 \\
\hline Mancuso F & 31 \\
\hline Marchetti C & 31 \\
\hline Marcuсci L & 5 \\
\hline Marozzo R & 28 \\
\hline Marrosu E & 44 \\
\hline Marzetti E & 18 \\
\hline Masiero S & 51 \\
\hline Mateescu GO & 52 \\
\hline Matheny $M$ & 42 \\
\hline Mehedinti $C M$ & 52 \\
\hline Meng $H$ & 34 \\
\hline Merlini $G$ & 45 \\
\hline Messina $G$ & 48 \\
\hline Michaud J & 41 \\
\hline Michelucci A & 16,28 \\
\hline Miranti CK & 34 \\
\hline Missaglia S & 49 \\
\hline Monet A & 6 \\
\hline Montanaro F & 44 \\
\hline Monti E & 10 \\
\hline Moresi V & 21 \\
\hline & \\
\hline
\end{tabular}

\begin{tabular}{|c|c|}
\hline Musci RV & 39 \\
\hline Narici $M$ & $3,5,10,25$ \\
\hline Negro F & 15 \\
\hline Odom G & 45 \\
\hline Ohlendieck K & 48 \\
\hline Paolini C & 16 \\
\hline Partridge TA & 30,33 \\
\hline Passaretti M & 29 \\
\hline Pasut A & 34 \\
\hline Pavan $P$ & 5,10 \\
\hline Pečlin P & 55 \\
\hline Pegoraro $V$ & 28 \\
\hline Pelizzola M & 50 \\
\hline Peterson CA & 34 \\
\hline Petrelli L & 4,6 \\
\hline Petronilli V & 45 \\
\hline Phelps MP & 38 \\
\hline Picca A & 18 \\
\hline Pietrangelo L & 28,59 \\
\hline Pietrangelo T & 24 \\
\hline Pirri $C$ & 4,6 \\
\hline Porzionato A & 4,6 \\
\hline Power GA & 8,9 \\
\hline Preti A & 48 \\
\hline Previtali SC & 48 \\
\hline Principi E & 48 \\
\hline Protasi F & $16,27,28,58,59$ \\
\hline Prüller J & 38 \\
\hline Puri PL & 50 \\
\hline Rachin A & 52 \\
\hline Raffaello A & 56 \\
\hline Raffaghello L & 48 \\
\hline Ramos $J$ & 6 \\
\hline Rastelli $G$ & 16 \\
\hline Reane Vecellio D & 56 \\
\hline Recchia D & 48 \\
\hline Recenti M & 6 \\
\hline Reggiani C & $5,10,56$ \\
\hline Regnier $M$ & 45 \\
\hline Ricciardi C & 6 \\
\hline Richard I & 43 \\
\hline Ritzmann $R$ & 10 \\
\hline Riuzzi F & 21,31 \\
\hline Rizzuto $R$ & 59 \\
\hline Rodia MT & 28 \\
\hline Rosati R & 54 \\
\hline Rovere-Querini P & 48 \\
\hline Rozman $J$ & 55 \\
\hline Rubartelli A & 48 \\
\hline Ruggieri E & 48 \\
\hline Ryan TE & 23,24 \\
\hline Sabatelli $P$ & 45 \\
\hline Sacchetto $R$ & 47 \\
\hline Saclier M & 48 \\
\hline Saini SK & 18 \\
\hline Salim de Castro $G$ & 21 \\
\hline Salvadori $L$ & 31 \\
\hline Sandonà D & 47 \\
\hline
\end{tabular}




$\begin{array}{llll}\text { Sanson } M & 43 & \text { Stocco A } & 45 \\ \text { Santi S } & 28 & \text { Stoessel SJ } & 39 \\ \text { Scano } M & 47 & \text { Stuelsatz P } & 41 \\ \text { Schaefer } L V & 16,17,18 & \text { Suel L } & 43 \\ \text { Scheib } M & 39 & \text { Surdu } \text { O } & 52 \\ \text { Schiavone } M & 45 & \text { Surdu V } & 52 \\ \text { Scott CS } & 44 & \text { Swandulla D } & 48 \\ \text { Seelaender } M & 21 & \text { Sweeney HL } & 42 \\ \text { Serano } M & 16,28 & \text { Tajbakhsh S } & 37 \\ \text { Servais } L & 43 & \text { Talbert E } & 19 \\ \text { Shao A } & 36 & \text { Tavian D } & 49 \\ \text { Shapiro JB } & 39 & \text { Thome T } & 23 \\ \text { Sileikyte } J & 45 & \text { Tirone } M & 29,48 \\ \text { Sinha Sh } & 12 & \text { Todros S } & 5 \\ \text { Sinha } U & 12 & \text { Tognolo L } & 51 \\ \text { Smolina } N & 45 & \text { Torchio S } & 48 \\ \text { Smuder AJ } & 22 & \text { Torcinaro A } & 50 \\ \text { Soardi } M & 47 & \text { Toth MJ } & 23 \\ \text { Sorci } G & 21,31 & \text { Trask AJ } & 44 \\ \text { Sotiropulos A } & 21 & \text { Trollet C } & 42 \\ \text { Southard SM } & 35 & \text { Tsika R } & 35 \\ \text { Stecco C } & 4,6 & \text { Vecellio RD } & 56 \\ & & & \end{array}$

$\begin{array}{ll}\text { Vénéreau E } & 29,48 \\ \text { Verratti V } & 24 \\ \text { Villa F } & 34 \\ \text { Voit T } & 43 \\ \text { Waldvogel J } & 10 \\ \text { Wang H } & 44 \\ \text { Wasala NB } & 44 \\ \text { White JC } & 12 \\ \text { Widrick J } & 34 \\ \text { Wright A } & 34 \\ \text { Xue Z } & 21 \\ \text { Yablonka-Reuveni Z } & 1,32,36 \\ \text { Yamamoto M } & 39 \\ \text { Yue Y } & 44 \\ \text { Zambrano S } & 29 \\ \text { Zammit PS } & 38 \\ \text { Zhang L } & 44 \\ \text { Zini N } & 28 \\ \text { Zofkie W } & 35 \\ \text { Zulian A } & 45\end{array}$

\section{Acknowledgments}

The Organizers thank Speakers for scientific and financial support of the 2021 PDM3 May 26-29, 2021.

\section{Funding}

E-publishing of this typescript is supported by the Armando \& Carmela Mioni-Carraro Foundation for Translational Myology, Padova, Italy and by PAGEpress, Pavia, Italy.

\section{Conflict of Interest None.}

\section{Ethical Publication Statement}

The Author confirms that he has read the Journal's position on issues involved in ethical publication and affirms that this report is consistent with those guidelines.

\section{Corresponding Author}

Ugo Carraro, Department of Biomedical Sciences, University of Padova, Italy.

ORCID iD: 0000-0002-0924-4998

Email: ugo.carraro@unipd.it

\section{Email and ORCID iD of Coauthor}

Zipora Yablonka-Reuveni: reuveni@u.washington.edu ORCID iD: 0000-0002-8855-263X

\section{References}

1. Carraro U. 2017Spring PaduaMuscleDays, roots and byproducts. Eur J Transl Myol. 2017 Jun 27;27(2):6810. doi: 10.4081/ejtm.2017.6810.

2. Carraro U. Exciting perspectives for Translational Myology in the Abstracts of the 2018Spring PaduaMuscleDays: Giovanni Salviati Memorial Chapter I - Foreword. Eur J Transl Myol. 2018 Feb 20;28(1):7363. doi: 10.4081/ejtm.2018.7363

3. Carraro U. Collection of the Abstracts of the 2019Spring PMD: Translational Myology and Mobility Medicine. Eur J Transl Myol. 2019 Mar 11;29(1):8155. doi: 10.4081/ejtm.2019.8155

4. Carraro U. Thirty years of translational research in Mobility Medicine: Collection of Abstracts of the 2020 Padua Muscle Days. Eur J Transl Myol 2020;30:3-47. Doi: 10.4081/ejtm.2019.8826

5. Carraro U. 30 Years of Translational Mobility Medicine: 2020 Padua Muscle Days go virtual from Euganean Hills, November 19th to 21st. Eur J Transl Myol. 2020 Nov 17;30(4):9437. doi: 10.4081 /ejtm.2020.9437.

Submitted: March 10, 2021 Accepted: March 17, 2021 